\title{
Electronic Nose-Based Fusarium Detection and Deoxynivalenol Aptamer Development
}

\author{
Dissertation \\ zur Erlangung des Doktorgrades \\ der Fakultät für Agrarwissenschaften \\ der Georg-August-Universität Göttingen
}

vorgelegt von

Jakob Eifler

geboren in Zittau

Göttingen, Juli 2014 
D7

1. Referent: Prof. Dr. Dr. Detlev Schild

2. Koreferent: Prof. Dr. Elke Pawelzik

Tag der letzten Prüfung: 23.07.2014 


\begin{abstract}
Fungal infestation on wheat is an increasingly grave nutritional problem in many countries worldwide. Fusarium species are especially harmful pathogens due to their toxic metabolites. Deoxynivalenol (DON) in particular, is a secondary metabolite that contaminates frequently cereal grain, cereal-based food or feed and is thus a serious health risk to humans. To comply with governmental regulations, new tools for fast and accurate DON and Fusaria estimation have to be developed. The discrimination properties of electronic noses have already been shown and are promising with respect to food control. Aptamers for ligand binding are already known and are suitable as recognition elements for biosensors.

In this work the volatile compounds released by Fusarium cerealis, Fusarium graminearum, Fusarium culmorum and Fusarium redolens were studied. Using an electronic nose, it was possible to differentiate between infected and non-infected dry, whole wheat grain. The electronic nose was capable of distinguishing between four Fusaria species with an accuracy higher than $80 \%$. Furthermore, DNA aptamers have been selected, reacting to DON with different affinities. The used SELEX is based on the formation of aptamer beacons upon DON binding. A single-stranded DNA library, covering 40 random positions, was bound to an affinity column. The library consisted of an estimated diversity of $6.6 \times 10^{14}$ molecules. Sequences which underwent a conformational change consequently to the addition of DON were collected and amplified. Eventually, 39 DNA structures of the amplified pool were chosen for binding analysis among which 14 reacted to the application of DON.
\end{abstract}




\section{Contents}

List of Figures ix

List of Tables $\quad$ xi

Nomenclature $\quad$ xiii

1 Introduction 1

1.1 The Genus Fusarium . . . . . . . . . . . . . . . 1

1.1.1 Occurrence and Propagation . . . . . . . . . . . 1

1.1.2 Fusarium Mycotoxins . . . . . . . . . . . . 3

1.1.2.1 Fumonisins . . . . . . . . . . . . 3

1.1.2.2 Zearalenone .............. 4

1.1.2.3 Trichothecenes . . . . . . . . . . . . 5

1.1.3 Volatiles Released by Fusarium . . . . . . . . . . 5

1.1.4 Analysis of Fusarium Infestation . . . . . . . . . 7

1.2 Characteristics of Deoxynivalenol . . . . . . . . . 8

1.2.1 Properties and Toxicology . . . . . . . . . . . 8

1.2.2 Grain Contamination . . . . . . . . . . . . . 99

1.2 .3 DON Analytics . . . . . . . . . . . . . . . 12

1.3 Electronic Nose . . . . . . . . . . . . . . . 13

1.4 Aptamers . . . . . . . . . . . . . . . 16

1.5 Scope of This Work . . . . . . . . . . . . . . . . 21

2 Materials and Methods 23

2.1 Samples . . . . . . . . . . . . . . . . . . 23 
2.1 .1 Grain Samples . . . . . . . . . . . . . . 23

2.1 .2 Oligonucleotides . . . . . . . . . . . . . . . 24

2.2 Volatile Analysis . . . . . . . . . . . . . . . 25

2.2.1 Headspace Generation . . . . . . . . . . . 25

2.2.2 Gas Chromatographic Analysis . . . . . . . . . 25

2.2 .3 Electronic Nose Analysis . . . . . . . . . . . . . . 26

2.3 Fluorescence Measurements and Fluorophores . . . . . . . . . 27

2.4 Aptamer Generation . . . . . . . . . . . . . . . 29

2.4.1 Optimisation of Capture Oligonucleotide . . . . . . . . 29

2.4.2 Optimisation of PCR Parameters . . . . . . . . . . 29

2.4.3 Optimisation of Strand Separation . . . . . . . . 30

2.4.4 Implementation of Column SELEX . . . . . . . . . . 30

2.4.5 Identifying Individual Aptamers . . . . . . . . . . . 35

2.5 Binding Assay . . . . . . . . . . . . . . 35

$\begin{array}{llr}3 & \text { Results } & 37\end{array}$

3.1 Electronic Nose-Based Detection of Fusarium spp. in Wheat Grain 37

3.1.1 Volatiles Released by Fusarium . . . . . . . . . . . 37

3.1.2 Species Identification . . . . . . . . . . . . . . 39

3.1.3 Classification of Infection Level . . . . . . . . . . . 41

3.1.4 Fungal Infection vs. Control . . . . . . . . . . . . . . 42

3.2 Selecting Aptamers against Deoxynivalenol . . . . . . . . . . . 45

3.2.1 Influence of Capture Oligonucleotide-Length on Binding Affinity ...................... 45

3.2.2 Optimisation of Amplification Procedure . . . . . . . . 47

3.2.2.1 PCR Parameters . . . . . . . . . . . . . 47

3.2.2.2 Double Strand Separation . . . . . . . . . 47

3.2.3 SELEX Experiment . . . . . . . . . . . . . 49

3.2.4 Characterisation of Selected Aptamers . . . . . . . . 51

4 Discussion $\quad 59$

4.1 Volatile Organic Compounds . . . . . . . . . . . . . . . 59

4.2 Electronic Nose Recognition . . . . . . . . . . . . . 60 


\section{CONTENTS}

4.3 Aptamer Development . . . . . . . . . . . . . . 62

4.4 DON Aptamers . . . . . . . . . . . . . . . . 66 66

4.5 Conclusions ........................ 68

$\begin{array}{lll}5 & \text { Summary } & 71\end{array}$

$\begin{array}{ll}\text { Bibliography } & 73\end{array}$

$\begin{array}{ll}\text { Acknowledgements } & 91\end{array}$ 


\section{List of Figures}

1.1 Fusarium contaminated wheat kernels ........... 2

1.2 Chemical structure of DON . . . . . . . . . . . 8

1.3 Potential setup of an electronic nose . . . . . . . . . . 16

1.4 SELEX procedure based on the development of aptamer beacons . 18

2.1 Fusarium-infected wheat kernels . . . . . . . . . . . . 24

2.2 Excitation and emission spectrum of 6 -HEX. . . . . . . . . . 28

2.3 Excitation and emission spectrum of SybrGreen I. . . . . . . . 28

2.4 Selection procedure for DON-aptamers . . . . . . . . . . 34

3.1 Temperature-dependency of abundances. . . . . . . . . . . 39

3.2 Enose results indicating different Fusarium species. . . . . . . . . 40

3.3 Enose results indicating different Fusarium infection levels. . . . . 41

3.4 Frequency shift of the quartz microbalance sensors . . . . . . . . . 43

3.5 Enose results indicating Fusarium infection . . . . . . . . . . . . 44

3.6 Optimisation of capture oligonucleotide length . . . . . . . . . 46

3.7 Estimating optimal PCR parameters . . . . . . . . . . . 48

3.8 Strand separation test . . . . . . . . . . . . . . . 49

3.9 Eluted ssDNA during different rounds of selection . . . . . . . . 50

3.10 Agarose gel electrophoresis of EcoR I digested pDNAs . . . . . . . 50

3.11 Binding assays with individual aptamers. . . . . . . . . . 52

3.12 Predicted secondary structure of clones c8, c15, c77 and c41 . . . 54

3.13 Predicted secondary structure of clones c32, c42, c29 and c18 . . 55

3.14 Binding assays of modified aptamers . . . . . . . . . . 56 


\section{List of Tables}

1.1 Mycotoxins produced by Fusarium species . . . . . . . . . . 4

1.2 Characteristics of DON . . . . . . . . . . . . . 9 9

1.3 DON-content of wheat grain in Germany . . . . . . . . . . . 10

1.4 Law-set maximum levels for DON . . . . . . . . . . . . . 12

1.5 Aptamers against small molecules. . . . . . . . . . . 19

2.1 Oligonucleotides . . . . . . . . . . . . . . 25

2.2 Input parameters of SELEX . . . . . . . . . . . . 32

2.3 Washing parameters of SELEX . . . . . . . . . . . 33

3.1 Volatile compounds released by the analysed fungi . . . . . . . 38

3.2 Confusion matrix of species classification . . . . . . . . . . . 40

3.3 Confusion matrix of classification of infection levels . . . . . . . 42

3.4 Confusion matrix of fungal infection vs. control . . . . . . . . . 42

3.5 Primary structure of DON binders . . . . . . . . . . 58 


\section{Nomenclature}

\begin{tabular}{|c|c|c|c|}
\hline $\mathrm{bp}$ & Base Pairs & i.p. & intraperitoneal \\
\hline \multirow{2}{*}{$\begin{array}{l}\text { bw } \\
\text { capDNA }\end{array}$} & Body Weight & \multirow[t]{2}{*}{ IUPAC } & \multirow{2}{*}{$\begin{array}{l}\text { International Union of Pure and } \\
\text { Applied Chemistry }\end{array}$} \\
\hline & Capture DNA & & \\
\hline $\mathrm{ct}$ & Threshold Cycle & i.v. & intravenous \\
\hline $\mathrm{CV}$ & Coefficient of Variation & $\mathrm{LD}_{50}$ & Median Lethal Dose \\
\hline$\Delta \mathrm{G}$ & Change in Gibbs Energy & $\mathrm{LV}$ & Latent Variable \\
\hline \multirow{4}{*}{$\begin{array}{l}\text { DM } \\
\text { DNA } \\
\text { dNTP }\end{array}$} & Dry Matter & \multirow[t]{2}{*}{ MVOC } & \multirow{2}{*}{$\begin{array}{l}\text { Microbial Volatile Organic } \\
\text { Compound }\end{array}$} \\
\hline & Deoxyribonucleic acid & & \\
\hline & Deoxyribonucleotide & NIRS & Near Infrared Spectroscopy \\
\hline & Triphosphate & PCR & Polymerase Chain Reaction \\
\hline DON & Deoxynivalenol & pDNA & Plasmid DNA \\
\hline \multirow{2}{*}{$\begin{array}{l}\text { DS } \\
\text { DSB }\end{array}$} & Disease Severity & \multirow[t]{2}{*}{ PLS-DA } & Partial Least Square \\
\hline & DON Selection Buffer & & Discriminant Analysis \\
\hline dsDNA & Double-Stranded DNA & p.o. & per os / orally \\
\hline Enose & Electronic Nose & QMB & Quartz Microbalance \\
\hline FHB & Fusarium Head Blight & RED & Relative Eluted DNA \\
\hline \multirow[t]{2}{*}{ GC/MS } & \multirow{2}{*}{$\begin{array}{l}\text { Gas Chromatography / } \\
\text { Mass Spectrometry }\end{array}$} & $\mathrm{RFU}$ & Relative Fluorescence Unit \\
\hline & & RNA & Ribonucleic acid \\
\hline \multirow[t]{2}{*}{ HEX } & \multirow{2}{*}{$\begin{array}{l}\text { Carboxy-2, } 4,4,5,7,7 \\
\text {-hexachlorofluorescein } \\
\text { Succinimidyl Ester }\end{array}$} & s.c. & subcutaneous \\
\hline & & SELEX & Systematic Evolution of Ligands \\
\hline \multirow[t]{2}{*}{ HPLC } & \multirow{2}{*}{$\begin{array}{l}\text { High Pressure Liquid } \\
\text { Chromatography }\end{array}$} & SDY & \\
\hline & & SPME & Solid Phase Micro Ex \\
\hline \multirow[t]{2}{*}{ IARC } & \multirow{2}{*}{$\begin{array}{l}\text { International Agency for } \\
\text { Research on Cancer }\end{array}$} & ssDNA & Single-Stranded DNA \\
\hline & & TDI & Tolerable Daily Intake \\
\hline
\end{tabular}




\section{Chapter 1}

\section{Introduction}

\subsection{The Genus Fusarium}

\subsubsection{Occurrence and Propagation}

Fusarium species are widespread pathogens in all grain-growing regions worldwide, and one of the most important genera of plant pathogenic fungi on earth $[1,2]$. They belong to the phylum Ascomycota, which is the largest phylum of fungi, with over 64,000 species [3]. Fusarium was first described by Link [4] and later classified by Wollenweber and Reinking in 1935 [5]. The genus Fusarium comprises more than 100 species with several varieties. It is pathogenic on major agricultural crops like maize, wheat, barley and other small grain, but also on fruits, stems, twigs, roots of various plants and in soils [6]. Fusarium is thus one of the most economically important fungus on earth. Moreover, it causes Fusarium Head Blight (FHB) on wheat, barley and other small grain. The occurrence of different Fusarium species is characteristic of FHB. Main inducers are F. graminearum, F. culmorum and F. avenaceum. F. poae, F. cerealis and $F$. equiseti are less frequently found, as well as other species which are sporadically involved. While the infestation often takes place with warm and humid weather conditions in the time of flowering, also agronomic factors and the plant genotype play a role in disease severity. If infested, pinkish-red mycelium develops on the spikelets, the kernels become shrivelled, with a white, pink or light brown appearance (cf. Figure 1.1) [1, 7]. Agronomical, FHB leads to a reduction 
in grain size, kernel weight, germination rate, as well as a depression in quality parameters $[1,8]$. Hence, the yield loss through FHB infection can be up to $70 \%$ [1]. Epidemics in China are most common and can affect more than 7 million hectares of wheat and a loss of up to 2.5 million tons of grain per year [9]. In the northern and central plains of the U.S., Nganje et al. [10] numbered the economic losses in wheat and barley to 2.7 billion dollars in the years 1998 to 2000. FHB has an impact on every sector of the wheat processing industry as it affects wheat growers, millers, bakers and consumers of cereal foodstuff.
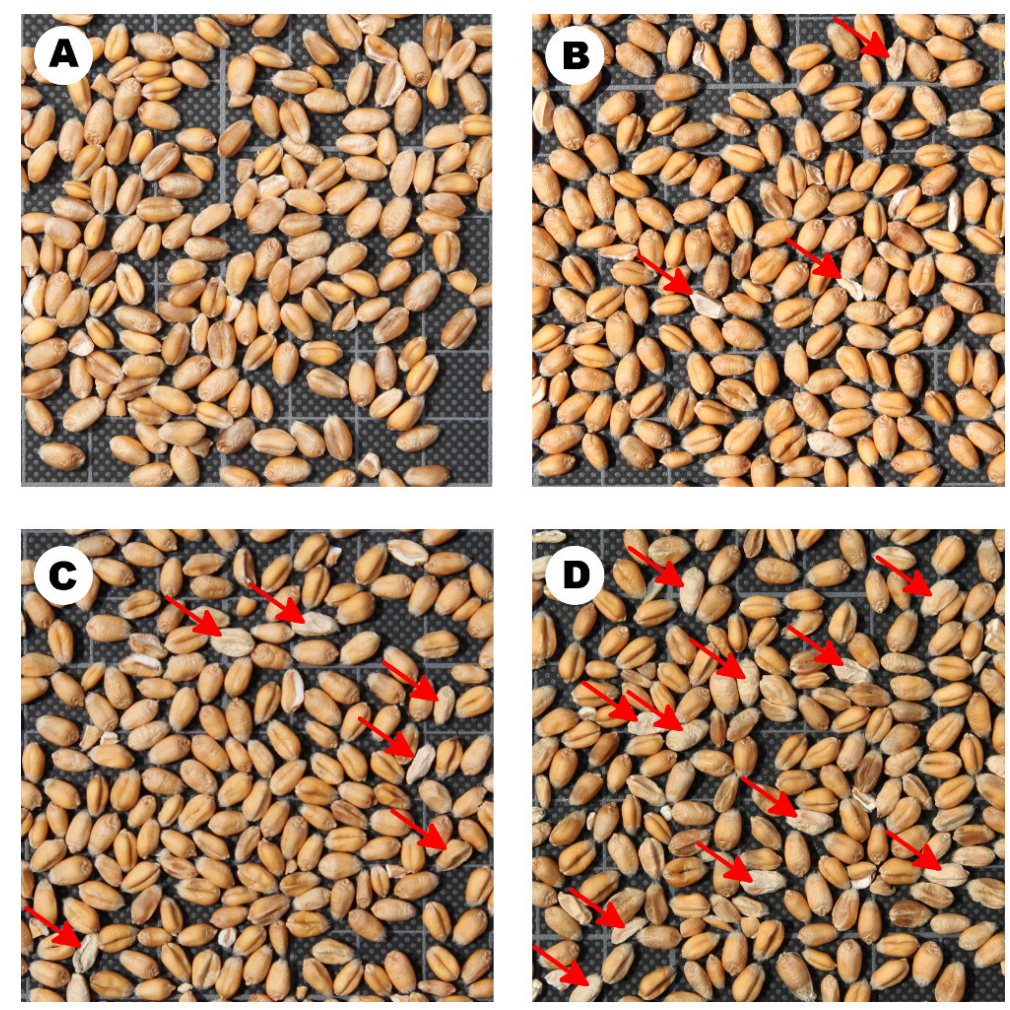

Figure 1.1: Fusarium-contaminated wheat kernels after harvesting, and the corresponding disease severity (DS) and DON-content. A: DS $0 \%$, DON $<0.5 \mathrm{mg} \mathrm{kg}^{-1} \mathrm{DM}$; B: DS $50 \%$, DON $2 \mathrm{mg} \mathrm{kg}^{-1} \mathrm{DM} ; \mathrm{C}:$ DS $65 \%$, DON $8 \mathrm{mg} \mathrm{kg}^{-1} \mathrm{DM}$; D: DS $70 \%$, DON $14 \mathrm{mg} \mathrm{kg}^{-1}$ DM. Arrows mark Fusariuminfected kernels. DM $=$ Dry Matter.

Fusarium causes on maize two major diseases known as Fusarium ear rot (pink ear rot) and Gibberella ear rot (red ear rot). The causal agent for Fusarium ear rot is mainly F. verticillioides, but also F. subglutinans and F. proliferatum. 


\section{INTRODUCTION}

In contrast, red ear rot is caused by F. graminearum and F. culmorum [11]. The diseases express themselves in the occurrence of mould with a white/light pink or red/pink colour and affect either single kernels/small groups of kernels (pink ear rot) or large portions of the ear, starting at the tip (red ear rot) [12]. While Fusarium ear rot is more common in warmer and drier areas, Gibberella ear rot is favoured by high levels of moisture and moderate temperatures [11]. Numerous field outbreaks of mycotoxicoses are known which were associated with contaminated maize used in livestock feed [13].

Fusarium disease control is challenging due to few fungicides available and the small application window. In Germany, there are accredited agents available for wheat (e.g. Prosaron, Bayer CropScience) and barley (Sportak 45 EW, BASF), but not for maize.

\subsubsection{Fusarium Mycotoxins}

Fusaria are producing several mycotoxins harmful to humans and vertebrates. The toxin production occurs mainly on the field in wet weather when harvests are delayed. Some species also produce mycotoxins during storage. Trichothecenes, specifically deoxynivalenol, zearalenone and fumonisins are found most frequently due to Fusarium infestation [7, 13]. In general, the amounts of metabolites in maize kernels are significantly higher than those in kernels of wheat or barley infected with the same fungal species [13]. Table 1.1 summarises the different mycotoxins recorded in cereals as a consequence of Fusarium spp. infection.

\subsubsection{Fumonisins}

Predominant producers of Fumonisins are F. verticillioides and F. proliferatum in maize. 16 fumonisins $\left(\mathrm{A}_{1}\right.$ to $\mathrm{A}_{4}, \mathrm{~B}_{1}$ to $\mathrm{B}_{4}, \mathrm{C}_{1}$ to $\mathrm{C}_{4}$ and $\mathrm{P}_{1}$ to $\mathrm{P}_{4}$ ) have been classified [15]. Among which the B-series is responsible for most cases of toxicosis caused by this class of toxins. Fumonisin $B_{1}$ is the most commonly identified mycotoxin in maize grain worldwide [13]. It causes outbreaks of equine leukoencephalomalacia [16] and porcine pulmonary edema syndrome [17, 18]. It is toxic to the central nervous system, liver, pancreas, kidney and lung in a number of animal species [18-20]. Esophageal cancer in humans has been correlated with 
Table 1.1: Mycotoxins produced by different Fusarium species. According to [7, $13,14]$.

\begin{tabular}{lcl}
\hline \multicolumn{1}{c}{ Species } & $\begin{array}{c}\text { Frequency } \\
\text { in cereals }\end{array}$ & \multicolumn{1}{c}{ Mycotoxin } \\
\hline F. graminearum & ++++ & DON, NIV, ZEA, AcDON, FUS \\
F. culmorum & +++++ & DON, ZEA, ZOH, NIV \\
F. avenaceum & +++++ & MON, ENS \\
F. poae & +++ & NIV, BEA, DAS, FUS, ENS \\
F. equiseti & ++ & DAS, ZEA, ZOH \\
F. tricinctum & ++ & MON \\
F. cerealis & & NIV, FUS, ZEA, ZOH \\
F. sporotrichioides & ++ & T2, HT2, T2ol, NEO \\
F. acuminatum & + & T2, NEO, MON \\
F. subglutinans & +++++ & $\mathrm{MON}, \mathrm{BEA}, \mathrm{FUB}$ \\
F. verticillioides & ++++ & $\mathrm{FB}_{1}, \mathrm{FB}_{2}, \mathrm{FB}, \mathrm{FU}-\mathrm{C}$ \\
F. redolens & ++ & $\mathrm{FB}_{1}, \mathrm{FB}_{2}, \mathrm{FB} 3$ \\
F. proliferatum & ++ & $\mathrm{FB}_{1}, \mathrm{FB}_{2}, \mathrm{MON}, \mathrm{BEA}$ \\
\hline
\end{tabular}

Frequency in European countries: +++++ very frequent to + extremely rare.

AcDON, monoacetyl-deoxynivalenols; BEA, beauvericin; DAS, diacetoxyscirpenol; DON, deoxynivalenol; ENS, enniatins; FB, fumonisin B; FUS, fusarenone-X; FUB, fusaproliferin; FU-C, fusarin; HT2, HT-2 toxin; MON, moniliformin; NEO, neosolaniol; NIV, nivalenol; T2, T2 toxin; T2ol, T-2 tetraol; ZEA, zearalenone; ZOH, zearalenols.

the consumption of $F$. verticillioides contaminated maize in Southern Africa [21] and China [22]. In rats, hepatocellular carcinomas were found after Fumonisin $\mathrm{B}_{1}$ intake [23]. Therefore, the International Agency for Research on Cancer (IARC) classified Fumonisin $\mathrm{B}_{1}$ and $\mathrm{B}_{2}$ in Group $2 \mathrm{~b}$ as possibly carcinogenic to humans $[24]$.

\subsubsection{Zearalenone}

Zearalenone is the most well-known substance of the group of resorcylic acid lactones. Superior producers are F. graminearum and F. culmorum. It was found in 1962, when F. graminearum contaminated corn was fed to swines [25], and later chemically characterised [26]. This toxin causes vulvar hypertrophy and vaginal eversion among the affected animals [25]. Furthermore, it has oestrogenic 
and genotoxic effects, and thus, has an anabolic impact on animal farming [27].

\subsubsection{Trichothecenes}

Trichothecenes belong to the sesquiterpenoid class and are commonly fungal secondary metabolites with to date more than 200 structurally related compounds known. They can be divided into macrocyclic (about 60\%) and non-macrocyclic (about 40\%) molecules [15]. All have a tricyclic 12,13-epoxytrichothec-9-ene group in common, which is essential for toxicity. Ueno classified them into four groups (type A-D) according to their chemical properties and producing fungi [28]. Type A and B are represented by Fusarium toxins e.g. T-2 toxin, HT-2 toxin, neosolaniol, diacetoxyscirpenol (type A) and e.g. nivalenol, deoxynivalenol, fusarenone-X, acetyldeoxynivalenol (type B). Type C and D toxins are comprising substances with a second epoxy compound (type C) and the group of macrocyclic molecules (type D). Trichothecenes have a wide range of biologic activity. Some represent the most toxic non-nitrogenous substances known [29]. Intraperitoneal $\mathrm{LD}_{50}$-values range from $0.5 \mathrm{mg} \mathrm{kg}^{-1} \mathrm{bw}$ (verrucarin) to $70 \mathrm{mg} \mathrm{kg}^{-1}$ bw (deoxynivalenol) $[28,30]$. Trichothecenes are known to inhibit eukaryotic and mitochondrial protein synthesis and interact with protein sulfhydryl groups [31]. Due to their small size, they can enter cells passively and can easily be absorbed via the gastrointestinal system [31]. They show antifungal [32], but also some carcinogenic activity [33]. Modified trichothecenes were used in chemotherapy due to their cytotoxicity to mammalian cells [34]. The potent acute toxicity and chemical stability qualify them as candidates for biologic warfare agents. Trichothecenes are speculated to have been used in Afghanistan and Asia in the 1970s. T-2 toxin, nivalenol and DON were identified in the vegetation. Additionally, T-2 toxin was found in the urine and blood samples of victims and in high concentration on rocks and gas masks [35].

\subsubsection{Volatiles Released by Fusarium}

Microbial volatile organic compounds (MVOCs) are produced in the primary and secondary metabolism of microorganisms such as fungi or bacteria. Among the frequent fungal MVOCs which have been reported are 2-methyl-1-propanol, 


\section{INTRODUCTION}

3-methyl-1-butanol, 1-octen-3-ol, 3-octanone, monoterpene and sesquiterpene [36]. Each organism releases thereby a composition of volatiles depending on its metabolism, growth phase and environmental conditions (nutrients, pH, humidity/water activity, temperature, ergosterol content of the growth substrate, ambient MVOCs in the air) [37-39]. The sensory analysis of fungal volatile metabolites by the human nose is the oldest and most commonly used method to detect spoilage of food or feed. In the 1970s the food-processing industry originally raised the analysis of MVOCs when biomarkers for food contamination as practical tools for analysis came up [39].

Fusaria have been shown to emanate a number of volatile compounds, specifically carbonyls, hydrocarbons, ketones, terpenes and complex mixtures of alcohols. These include common MVOCs such as 1-octen-3-ol, 3-methyl-1-butanol or 3-octanone [38, 40-43], as well as Fusarium spp. specific ones. Pasanen et al. [38] identified particularly terpenes and ketones as specific to Fusarium sporotrichioides grown on wheat kernels. Likewise mono- and sesquiterpene, often unknown ones, were reported to be released by F. culmorum [41] and F. sambucinum [44] as well as F. graminearum, F. avenaceum, F. poae [43] on wheat. A wellknown biomarker for Fusarium infection is trichodiene. It is generally related to trichothecene-producing fungi such as F. culmorum [45], F. graminearum [43, 46] and F. sporotrichioides, F. sambucinum, F. poae [46]. However, there is evidence that non-trichothecene producers also release trichodiene [47].

MVOCs can be collected from air by means of either dynamic or static sampling. In dynamic sampling the air is guided through an open- or closed-loop system and enriched in an adsorbing agent. In the static procedure the sample is air tight sealed in a glass vial or sample bag [48] and directly analysed or adsorbed on a sorbent as well. Several sorbents such as Tennax TA, Chromosorb, Carbotrap, Carbopack, Anasorb or Porasil C are broadly used [49]. The most frequent used technique in static sampling is the solid phase micro extraction (SPME) with polymer coated fused silica fibres. It is advantageous due to its speed and ease of use [50]. The volatiles can be released from the adsorbent material by thermal desorption or solvent extraction and are identified on a gas chromatograph, commonly coupled to a mass spectrometer or a flame ionisation detector. 


\subsubsection{Analysis of Fusarium Infestation}

The easiest and fastest method is to determine the disease severity visually. Under pre-harvest conditions, the infected spikelets per ear are counted, and the disease severity is calculated as follows: the number of infected spikelets times the percentage of infected surface divided by the total number of spikelets per ear. In the case of post-harvest analysis, visual assessment has to be done by counting Fusarium-damaged kernels from a random sample, based on morphological differences between infected grain and uninfected ones (cf. Figure 1.1). This process has been optimised by automatic image processing, in order to run the assessment commercially [51, 52]. However, visually assessing the degree of infestation is relatively imprecise, owing to the diverse appearances and the lack of species detection. Likewise, estimating fungal contamination by near infrared spectroscopy (NIRS) [53, 54] did not become accepted in practise. Current methods are either immunochemical or DNA-based. Enzyme linked immunosorbent assays (ELISA) often use fungal proteins or extracellular polysaccharides as antigen [55-57]. These assays are partly species specific, although they sometimes cross react with other fungal genera or other Fusarium species [55, 58]. A further disadvantage is the instability of the antigens. If the grain is processed at high temperatures during manufacture, the antigens can no longer be detected. The method of choice, when it becomes to accuracy and specificity in analysing Fusarium infestation is the measure of fungal specific DNA via the polymerase chain reaction (PCR) [59], since specific primers have been developed covering most of the Fusarium species [60]. This can be done either by conventional or quantitative PCR (qPCR), with the latter being highly, quantitatively sensitive [59]. The next evolutionary step in accuracy would be to opt for the "droplet digital PCR" (ddPCR) [61] offering highly precise and direct quantification [62]. However, the methods are laboratory-based, expensive, time-consuming and not applicable in the field. 


\subsection{Characteristics of Deoxynivalenol}

DON is frequently encountered in cereals and maize $[2,63]$ and it is the most important toxin of the group B trichothecenes. The first report of DON was in Japanese barley, isolated from Fusarium spp. in 1972 by Morooka et al. [64]. The authors called the substance "RD-toxin". It was later named deoxynivalenol, when Yoshizawa and Morooka discovered the structure [65]. At the same time, Vesonder et al. [66] isolated deoxynivalenol from U.S. corn in 1973 and labelled it as vomitoxin, according to its emetic effect.

\subsubsection{Properties and Toxicology}

Chemically, DON has a polar, organic structure (cf. Figure 1.2) and a molecular weight of $296.32 \mathrm{~g} \mathrm{~mol}^{-1}$. It is soluble in water, methanol and acetonitrile and has an UV-absorption with an extinction maximum at $218 \mathrm{~nm}$ (acetonitrile) to $219 \mathrm{~nm}$ (methanol). Table 1.2 is summarising the characteristics of DON. It is a relatively heat stable compound and does not degrade at high temperatures during cooking or baking [67]. If taken up by vertebrates, the metabolite which is most frequently found is de-epoxy-deoxynivalenol (DOM-1) [68-71].

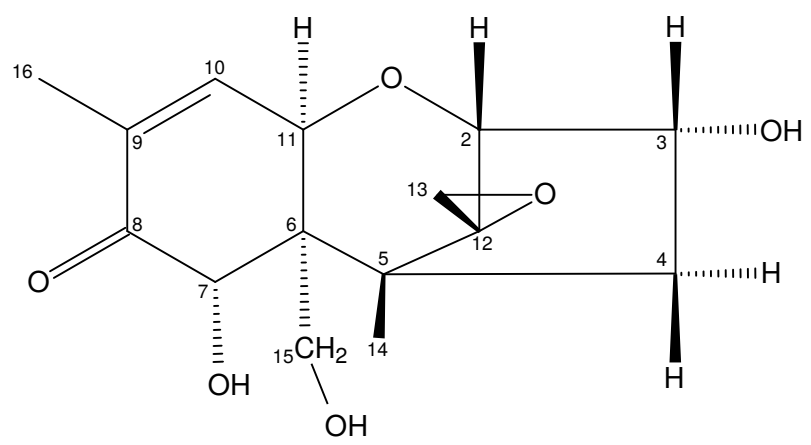

Figure 1.2: Chemical structure of deoxynivalenol [65].

Biochemically, DON has been shown to inhibit protein, DNA and RNA synthesis [75]. It can induce haemolysis of erythrocytes [76] and inhibit or induce apoptosis of lymphocytes in a dose-dependent manner [77]. DON is thus toxic to human and animal consumption. Extremely high doses can lead to death. $\mathrm{LD}_{50}$ 
Table 1.2: Characteristics of deoxynivalenol, as reported in several studies [24, 28, $72-74]$.

\begin{tabular}{|c|c|}
\hline Property & Value \\
\hline IUPAC Name & $3 \alpha, 7 \alpha, 15$-trihydroxy-12,13-epoxytrichotec-9-en-8-one \\
\hline Synonyms & $\begin{array}{l}\text { Vomitoxin, Dehydronivalenol, 4-Deoxynivalenol, RD- } \\
\text { Toxin }\end{array}$ \\
\hline Molecular Weight & $296.32 \mathrm{~g} \mathrm{~mol}^{-1}$ \\
\hline Molecular Formula & $\mathrm{C}_{15} \mathrm{H}_{20} \mathrm{O}_{6}$ \\
\hline Melting Point & 151 to $153^{\circ} \mathrm{C}$ \\
\hline Extinction Coefficient & 6.000 to $6.500 \mathrm{lmol}^{-1} \mathrm{~cm}^{-1}$ at $219 \mathrm{~nm}$ in Methanol \\
\hline \multirow[t]{3}{*}{ Toxicity } & Mouse $\mathrm{LD}_{50 \text { (p.o.) }}=46$ to $78 \mathrm{mg} \mathrm{kg}^{-1} \mathrm{bw}$ \\
\hline & Duckling $\mathrm{LD}_{50(\text { s.c. })}=27 \mathrm{mg} \mathrm{kg}^{-1} \mathrm{bw}$ \\
\hline & Broiler Chicks $\mathrm{LD}_{50 \text { (р.о.) }}=140 \mathrm{mg} \mathrm{kg}^{-1} \mathrm{bw}$ \\
\hline \multirow[t]{2}{*}{ Carcinogenicity } & IARC Group 3 \\
\hline & not classifiable as to its carcinogenicity to humans \\
\hline
\end{tabular}

values in mice range from 46 to $78 \mathrm{mg} \mathrm{kg}^{-1}$ bw for oral application [28, 73]. Symptoms are feed refusal, growth retardation, vomiting and diarrhoea. All animals are effected, most susceptible are swines $>$ mice $>$ rats $>$ poultry/ruminants [30]. In humans, intoxications associated with DON and other trichothecene consumption in China, Japan and India were reported. The symptoms found were nausea, abdominal pain, throat irritation, diarrhoea, dizziness and headache [75]. There is evidence that DON alters the neurochemistry of the brain, specifically the levels of biogenic monoamines in the central nervous system, causing feed refusal, emesis and anorexia. It is furthermore hypothesised that chronic DON-consumption will possibly lead to a long-term deregulation of the brain [78].

\subsubsection{Grain Contamination}

The DON contamination of grain is an increasing problem worldwide. In 2001 the WHO/FAO carried out a world-wide study comprising Argentina, Brazil, Canada, China, Finland, Germany, Italy, the Netherlands, Norway, Sweden, the United Kingdom, Uruguay and the USA. It was estimated therein that, on average, $57 \%$ 
of wheat (11,444 samples analysed), $68 \%$ of oat (834), $59 \%$ of barley $(1,662)$ and $41 \%$ of maize $(5,349)$ were contaminated with DON [63]. Schothorst et al. [79] assessed in 2004 similar results for 11 European countries in which $57 \%$ of 11,022 samples analysed were positive to deoxynivalenol. Results from a random analysis in Germany indicate that only $29 \%$ of all cereal-based products are devoid of Fusarium-toxins [80]. Moreover, the absolute DON contamination is alarming. Exceedingly high DON values were found in maize grain and cobs in Poland, reaching up to $927 \mathrm{mg} \mathrm{kg}^{-1}$ [81]. Possibly harmful concentrations (up to $71 \mathrm{mg} \mathrm{kg}^{-1}$ ) were reported from Argentina, Canada, Germany, Japan, New Zealand, Norway, Poland and the USA in wheat and barley [81]. However, the measures were often collected within a single year and DON mean levels in cereal grain are fluctuating heavily over the years. This is a result of the strong correlation between Fusarium spp. infection and variations in weather from one year to another. Nonetheless, the number of positive samples strongly increased in the last few years, which might also be due to a lower limit of detection (cf. Table 1.3).

Table 1.3: Comparison of DON-content of air dried wheat grain within Germany for the last 12 years [82].

\begin{tabular}{lcrccc}
\hline Year Samples & $\begin{array}{c}\text { Mean } \\
{\left[\mathrm{\mu g} \mathrm{kg}^{-1}\right]}\end{array}$ & $\begin{array}{c}\text { Min-Max } \\
{\left[\mathrm{\mu g} \mathrm{kg}^{-1}\right]}\end{array}$ & $\begin{array}{c}\text { Positive } \\
{[\%]}\end{array}$ & $\begin{array}{c}\text { Legal Limit } \\
{[\%]}\end{array}$ \\
\hline 2002 & 261 & 239 & $<10-3,616$ & - & - \\
2003 & 457 & 148 & $<10-2,692$ & - & - \\
2004 & 505 & 268 & $<10-3,965$ & - & - \\
2005 & 496 & 80 & $<10-4,097$ & - & - \\
2006 & 471 & 88 & $<10-7,543$ & - & - \\
2007 & 481 & 394 & $<10-12,249$ & - & - \\
2008 & 468 & 70 & $<5-2,506$ & 56 & $<1$ \\
2009 & 473 & 118 & $<5-7,236$ & 58 & 2 \\
2010 & 458 & 127 & $<3-5,005$ & 66 & 2 \\
2011 & 462 & 68 & $<3-2,024$ & 69 & 1 \\
2012 & 473 & 367 & $<3-29,266$ & 100 & 6 \\
2013 & 435 & 61 & $<3-1,711$ & 99 & $<1$ \\
\hline
\end{tabular}

The mycotoxin appears on the other hand to spread to food products, becoming a potential and presumably serious health risk to humans [83]. Accordingly, DON was frequently found in several commercial products including flour [84], 
pasta [85, 86], bread [85], beer [87] and cornflakes [88]. Many countries worldwide consequently issued guidelines and regulatory limits for Fusarium mycotoxins [89]. Table 1.4 outlines the law-set maximum levels for DON in cereals in several countries. The limits are lower for further processed cereals since those are already closer to the final product and thus the consumer. This results in a maximum level of $200 \mu \mathrm{g} \mathrm{kg}^{-1}$ dry matter for infant food in the European Union. Interestingly, commodities in the EU which are highly susceptible for Fusarium infestation and DON contamination are allowed to have a higher maximum DON concentration than others. However, based on these levels a tolerable daily intake (TDI) for DON of $1.0 \mu \mathrm{g} \mathrm{kg}^{-1}$ bw was set by the European Commission [90]. Nevertheless, for some groups of people, such as young children, DON daily intakes are very close to the TDI or above [79]. Besides, the toxin was found in the urine of adults from the United Kingdom, indicating a DON intake through food consumption. Wholemeal and white bread in particular was identified as sources of the toxin [91]. Experts from the grain-processing industry were asked in 2012 how the consumer could be better protected against unwanted DON uptake. The main finding was that DON-monitoring during grain delivery should be intensively improved by the use of rapid tests [92]. 
Table 1.4: Law-set maximum levels for Deoxynivalenol. After Regulation (EC) No $1881 / 2006$ and [93].

\begin{tabular}{llc}
\hline Country & Product & $\begin{array}{c}\text { Maximum levels } \\
{\left[\mu \mathrm{g} \mathrm{kg}{ }^{-1} \mathrm{DM}\right]}\end{array}$ \\
\hline European Union & unprocessed cereals of durum wheat, oat, & 1750 \\
& maize & 1250 \\
& other unprocessed cereals & 750 \\
& cereal flour, pasta & 500 \\
& cereal foodstuff & 200 \\
\hline Switzerland & cereal products & 500 \\
\hline Canada & uncleaned soft wheat & 2000 \\
& flour & 1200 \\
& uncleaned soft wheat for infant food & 1000 \\
& flour for infant food & 600 \\
\hline United States & finished wheat products & 1000 \\
\hline Russia & wheat cereals, flour & 1000 \\
\hline DM=dry matter & & \\
& &
\end{tabular}

$\mathrm{DM}=$ dry matter

\subsubsection{DON Analytics}

The analytics of DON from grain or food samples is based on the extraction method, the clean-up of the extract and the mycotoxin detection. There are several method available for each of these steps. As DON is water soluble the extraction could principally be done by water-extraction. In practice often wateracetonitrile, water-methanol or a combination of both is used. Acetonitrile has been shown to give cleaner extracts [94]. Additionally, a mixture including acetonitrile or ethanol allows simultaneous extraction of several trichothecenes and other mycotoxins. The clean-up procedure is necessary for removing interfering, co-extracted contaminates. Some widely used methods are the clean-up with immunoaffinity columns (IAC) [95-97] or solid-phase-extractions (SPE) [98, 99]. Particularly, "Mycosep" columns (RomerLabs, Washington, MO, USA) are broadly employed [100, 101]. The most common methods for the actual analysis of DON use either liquid-chromatography (LC) [99, 101, 102] or gas-chromatography (GC) [103, 104], coupled to a mass- or UV-absorbance spectrometer. Moreover, 


\section{INTRODUCTION}

also thin layer chromatography (TLC) is sometimes employed due to the small costs and effort [105]. Likewise, immunoassays (ELISA) [105, 106] provide a fast and cheap alternative to the bench-top, laboratory methods. In addition, they do not need necessarily a clean-up procedure. The detection limits of these methods for the analysis of DON in cereals range from 1 to $500 \mathrm{ng} \mathrm{g}^{-1}$ [107]. However, in an interlaboratory comparison based on identical standards, the DON-ELISAtestkits did not show good accuracy. The best results were obtained with a Mycosep clean-up and chromatographic detection [108].

\subsection{Electronic Nose}

The first reports of electronic gas sensors arose in the 1950s/1960s, when microelectrodes were used to generate an electrical signal upon volatile interaction [109, 110]. Persaud and Dodd [111] created in 1982 an intelligent array system of three chemosensitive gas sensors for pattern recognition as a model nose for the mammalian olfactory system. The term "Electronic Nose" (Enose) was later defined as a system of electronic chemical sensors recognising different volatiles and odours [112]. The biologic process of olfaction in vertebrates starts in the main olfactory epithelium, which is located in the nasal cavity. Membrane proteins of olfactory receptor neurons, placed in the mucous layer of the olfactory epithelium, recognise the complex composition of molecules from a particular odour. Upon detection, action potentials are generated and transmitted via the olfactory nerve to the olfactory bulb. From there the information is conveyed through different layers of processing to the higher brain [113]. Each receptor is thereby sensitive to a certain range of molecules. Similarly, in an electronic nose sensor surfaces of an array of different sensors react to gas molecules and produce electronic outputs, which are amplified and relayed to a processing unit. This approach is not fully comparable, since the mammalian nose has hundreds of different receptors which are continuously regenerated. However, electronic nose sensors can be even more specific than biological ones, in that they additionally offer the ability to sense non-odorant molecules (e.g. carbon monoxide).

Chemical sensors are devices which transpose chemical information into analytical useful signals [114]. According to Gründler [115], each sensor needs to have 


\section{INTRODUCTION}

a certain selectivity, which means that it should respond to the target substance within a certain time and distinguish it from the background. The sensors should furthermore exhibit stability and should recover in a short time period. Sensors usually have two functional units, the receptor and the transducer. While the receptor transforms the specific, concentration-dependent chemical information in a measurable form of energy, the transducer evaluates the energy and outputs an analytical signal [114]. Several gas sensor types were characterised according to the different receptor and transducer principles, such as metal-oxide sensors, conducting polymers, surface/bulk acoustic wave sensors, field effect sensors or electrochemical sensors [116].

Metal-oxide sensors are widely used and work with a semiconducting metaloxide surface, which is most often from $\mathrm{SnO}_{2}$ or $\mathrm{ZnO}$. Besides other oxidematerials, specifically $\mathrm{TiO}_{2}$ or $\mathrm{Fe}_{2} \mathrm{O}_{3}$ have been used. The working principle is based on the alternating conductance of the palladium- or platinum-doped surface, when interacting with gas molecules. At high temperatures $\left(>200{ }^{\circ} \mathrm{C}\right)$ the surface adsorbs molecules of oxygen in fairly fast rate of reaction. These molecules abstract electrons from the semiconducting surface, causing a conductance drop. The analytes react accordingly with the charged oxygen molecules in a redox reaction, subsequently releasing electrons and leading to an increase in conductance. Metal-oxide sensors are relative stable with a low humidity dependence. They are not highly selective and react to various volatiles [116]. Among the most frequently operated and commercially sold sensors are the Taguchi Gas Sensors (TGS) from Figaro Engineering Inc. (Japan). They provide sensors for the detection of combustible gases (methane, propane, hydrogen), toxic gases (carbon monoxide, ammonia, hydrogen sulfide), volatile organic vapours and refrigerant gases in a working range of 5 to $10,000 \mathrm{ppm}$ [117].

Another notable sensor type makes use of the piezoelectric effect and is referred to as the quartz crystal microbalance (QMB). It is composed of a monocrystal quartz which usually makes contact with gold electrodes. Upon excitation, the quartz oscillates with a certain resonance frequency typically in the range of 5 to $20 \mathrm{MHz}$ [116]. By coating the surface with a thin acceptor layer, the resonant frequency is changing according to Sauerbrey [118], due to the increased mass load. A further change of frequency $\Delta f$ can be observed, if an analyte binds to 
the acceptor layer, leading to an additional mass load $\Delta m$. By measuring $\Delta f$ the load can be estimated according to Equation 1.1 [118], provided that the density of the quartz $\rho_{Q}$, the surface area $A$, the thickness $d$ and the eigenfrequency $f_{0}$ are known. The quantities $\Delta m$ and $\Delta f$ are thus linearly related to each other in the low-perturbation regime [119]. As frequency measurements have a typical resolution of around $1 \mathrm{~Hz}$, weights of a few nanograms can be estimated precisely.

$$
\Delta m=-\frac{\rho_{Q} \cdot A \cdot d \cdot \Delta f}{f_{0}}
$$

Many different coating materials such as palladium, platin, gold [115], polymeric material [120, 121] or metalloporphyrins [119] have been used. The selectivity is highly dependent on the coating material and thus can be regulated in a proper way. Another advantage is their ambient working temperature, avoiding oxidising effects. Nevertheless, they are only rarely used in commercial electronic noses, due to their slow recovery and short lifetime from less than two years [122].

In addition to the sensor array, an electronic nose contains devices for sampling, odour delivery, signal acquisition and data processing [114]. Figure 1.3 shows the potential setup of an electronic nose for static headspace sampling of grain samples.

Electronic nose data analysis starts with data acquisition. Time-dependent responses from the transducers have to be collected within a signal pattern vector. The second step is feature extraction. Essentially, it is a dimensionality reduction process. Features, which were often applied, are the maximum sensor responses, temporal data or mapping features. Furthermore, normalisation and standardisation techniques are used to avoid scaling effects. At this stage, relative scaling, noise reduction, and baseline subtraction are common. Eventually, pattern recognition is generally done by multivariate data analysis. It can be divided into statistical parametric analysis beneath principal component analysis (PCA), partial least squares (PLS) or k-means algorithm and biological inspired artificial neural networks like multi-layer perceptions (MLP) or sammon mapping (SOM), amongst others [116, 124].

So far, electronic noses have been used in agriculture for a wide variety of 


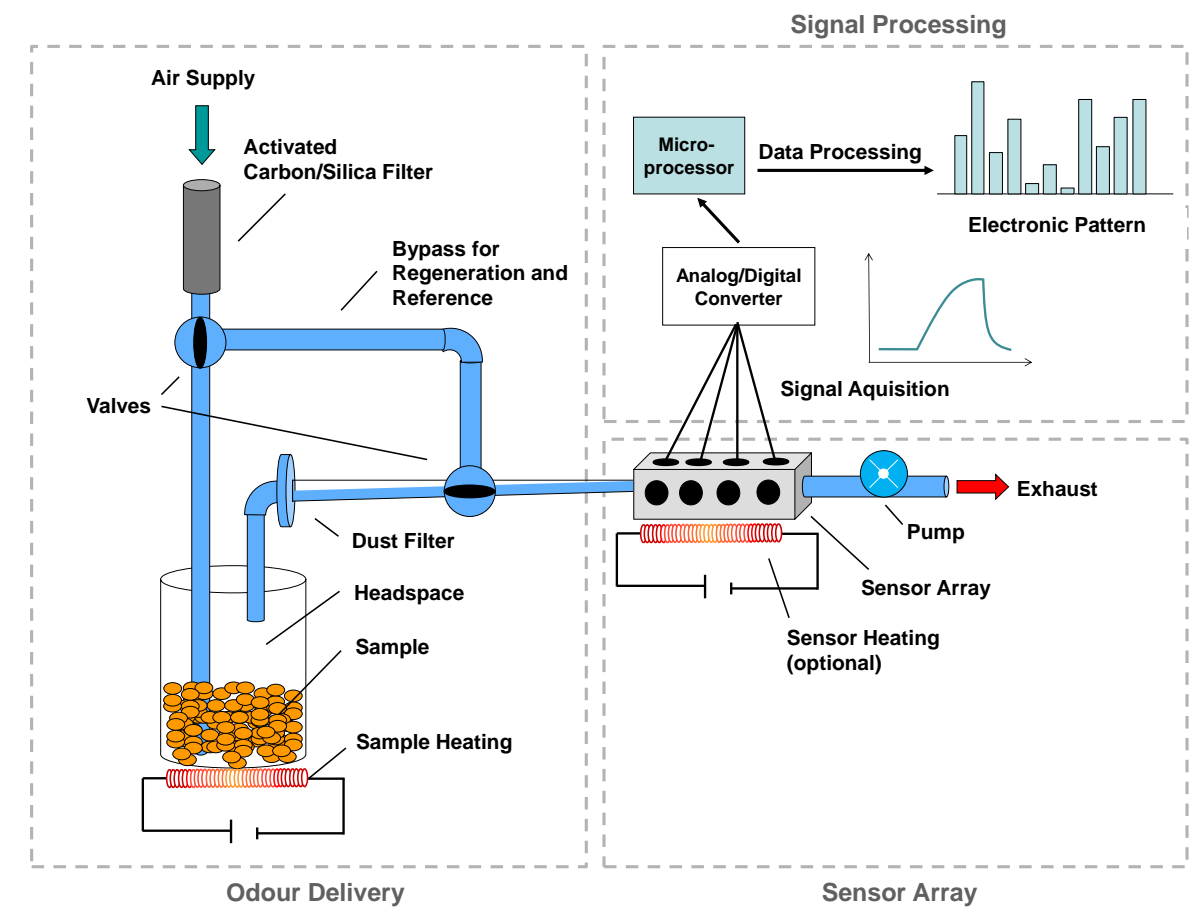

Figure 1.3: Potential setup of an electronic nose for static headspace sampling of grain samples. Modified after [123].

applications, including post-harvest quality assessment of oranges, apples and wine grapes [125, 126], discriminating flavours from coffee or beer [127, 128] and the detection of fungal spoilage and mycotoxins of grain [129-133].

\subsection{Aptamers}

It was found in the 1960s that nucleic acids can fold into complex three-dimensional structures and interact in biological processes [134]. Later it was shown that non-coding RNA as well as DNA display catalytic or binding properties [135, 136]. In 1990 Ellington and Szostak [137] termed ligand-binding RNAs, "Aptamers". Nowadays, aptamers are generally small oligonucleotides of 30 to 150 bases of either RNA or single-stranded DNA binding specific ligands. Several of their properties are superior to those of antibodies. The target binding site of aptamers can be specifically selected in an in vitro selection under a variety of 


\section{INTRODUCTION}

conditions. Thus, the binding site is not restricted by the immune system and not limited to physiological conditions. Aptamers can be easily chemically modified at clearly defined positions (e.g. for the inclusion of dyes or reporter molecules). Eventually, they have an unlimited shelf-life, are reverse denaturable and there is no evidence for immunogenicity $[138,139]$. As they are besides small in size $(<50 \mathrm{kDa})$, they are suitable as labelling probes in fluorescence microscopy [140].

Aptamers are selected through systematic evolution of ligands by exponential enrichment (SELEX). This procedure was first described by Tuerk and Gold [141] in 1990. It is based on variation, selection and replication. Thus, a random, combinatorial library of single-stranded oligonucleotides is exposed to a target molecule. The binding sequences are separated and multiplied, forming a new library which is then, in a new cycle again exposed to the target. Multiple rounds of selection and enrichment are leading to an exponential increase of the best binding species. The crucial point of the selection is the separation of the bound species from the unbound ones and this efficiency determines the number of cycles necessary. The separation is highly dependent on the target properties, especially size and charge. If the target-aptamer complex is much bigger than the individual aptamers of the library, filters can be used to fulfil this step [141]. If not, targets are often immobilised on a matrix which eventually leads to capture of bound oligonucleotides, and thus, to a separation (e.g. FluMag SELEX [142]). While this method has been successfully used for a bunch of large targets, it is challenging, especially for small molecules as deoxynivalenol, due to a deficiency in functional groups and contact surface. One possibility to treat this problem is the immobilisation of the library and the selection due to the development of aptamer beacons [143] (referred to as column-SELEX). Here, the library is bound to an affinity column by hybridising to a single-stranded capture oligonucleotide which is partially complementary to one of the constant regions. The capture oligonucleotide is furthermore strongly bound via a biotin-link to a streptavidin-agarose column. The biotin-streptavidin interaction is, with a dissociation constant in the order of $1 \times 10^{-15} \mathrm{M}$ [144], one of the strongest non-covalent biological bonds known. The target molecule is now able to freely interact with the matrix-bound library. When the target binds to a specific sequence, the DNA-species undergoes a conformational change by forming a beacon and is thus released from the 


\section{INTRODUCTION}

column. The eluted species are collected and amplified to form a new pool for a further round of selection. (cf. Figure 1.4). As the relevant species are amplified every round, an exponential increase can be observed. The selected DNA can be analysed and the pertinent species can be chosen for further investigations.

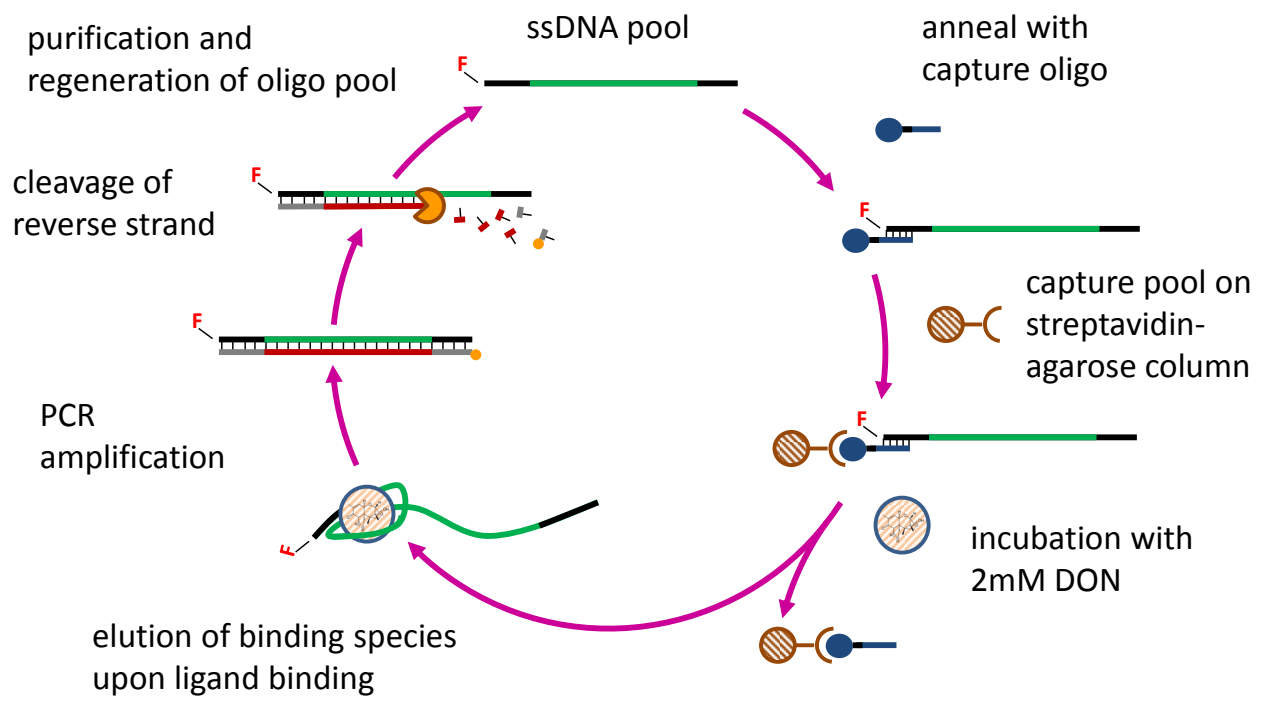

Figure 1.4: SELEX procedure based on the development of aptamer beacons. The target interacts with immobilised oligonucleotides from an affinity column and results in a target-initiated elution of binding species. Modified after [145].

Aptamers have been discovered for a wide variety of different ligands, such as proteins and large molecules. Very few data are available for the selection against small molecules, such as nucleobases/-tides, amino acids, antibiotics or mycotoxins. An overview of aptamers selected against small molecules so far, is given in Table 1.5.

It is predicted that aptamers have a high value impact on diagnosis and therapeutics in the future [173]. However, up to now, they have slowly reached the marketplace, with only one aptamer being approved as drug. In $2004 \mathrm{Pe}-$ gaptanib (tradename Macugen, Novartis AG, Basel, $\mathrm{CH}$ ) was released for the treatment of age-related macular degeneration (AMD) [174]. Several other candidates are in clinical studies, such as REG1 to REG3 from RegardoBiosciences (New Jersey, USA) [175] against cardiovascular disease, or the spiegelmers Emapticap Pegol, Olaptesed Pegol and Lexaptepid Pegol for the treatment of cancer or 
Table 1.5: Aptamers against small molecules.

\begin{tabular}{|c|c|c|c|c|c|}
\hline Target & $\begin{array}{c}\mathbf{M} \\
\left(\mathrm{g} \mathrm{mol}^{-1}\right)\end{array}$ & $\begin{array}{c}\mathbf{K}_{\mathbf{d}} \\
(\mu \mathrm{M})\end{array}$ & Тур & $\begin{array}{c}\text { Length }^{1} \\
\text { (nt) }\end{array}$ & Reference \\
\hline \multicolumn{6}{|c|}{ Nucleobases/Nucleosides/Nucleotides } \\
\hline Adenine & 135 & 10 & RNA & 52 & [146] \\
\hline Xanthnine, Guanine & 152,151 & 3.3 & RNA & & {$[147]$} \\
\hline Adenosine & 267 & 6 & DNA & 27 & {$[148]$} \\
\hline cAMP & 329 & 10 & RNA & $31-33$ & [149] \\
\hline ATP & 507 & 0.7 & RNA & 27 & {$[150]$} \\
\hline GTP & 523 & $0.025-0.5$ & RNA & 39 & {$[151]$} \\
\hline ATP, GTP & 507,523 & & DNA & 49 & {$[152]$} \\
\hline \multicolumn{6}{|l|}{ Amino Acids } \\
\hline Isoleucine & 131 & $200-500$ & RNA & 95 & {$[153]$} \\
\hline L-Histidine & 155 & $8-54$ & RNA & 70 & {$[154]$} \\
\hline L-Arginine & 174 & 0.33 & RNA & & [155] \\
\hline L-Citrulline & 175 & $62-68$ & RNA & 44 & {$[156]$} \\
\hline \multicolumn{6}{|l|}{ Antibiotics } \\
\hline Tobramycin & 468 & $0.77 \mathrm{nM}$ & RNA & 109 & {$[157]$} \\
\hline Kanamycin B & 484 & 0.18 & RNA & & [158] \\
\hline Streptomycin & 582 & & RNA & 74 & [159] \\
\hline \multicolumn{6}{|l|}{ Mycotoxins } \\
\hline Aflatoxin $\mathrm{B}_{1}$ & 312 & 0.011 & DNA & & [160] \\
\hline Ochatoxin A & 404 & $0.2-1.4$ & DNA & 40 & {$[161,162]$} \\
\hline Fumonisin $\mathrm{B}_{1}$ & 722 & 0.1 & DNA & 60 & {$[163]$} \\
\hline \multicolumn{6}{|l|}{ Others } \\
\hline $\mathrm{K}^{+}$ & 39 & 500 & DNA & 21 & [164] \\
\hline $\mathrm{Ni}^{2+}$ & 59 & 1 & RNA & 95 & {$[165]$} \\
\hline $\mathrm{Zn}^{2+}$ & 65 & 15 & DNA & 50 & {$[145]$} \\
\hline Dopamine & 153 & 2.8 & RNA & 114 & [166] \\
\hline Biotin & 244 & 5.7 & RNA & 100 & {$[167]$} \\
\hline Cocaine & 303 & $0.4-10$ & DNA & 38 & [168] \\
\hline Riboflavin & 376 & $1-5$ & RNA/DNA & 72 & [169] \\
\hline Daunorubicin & 528 & 0.02 & DNA & 40 & [170] \\
\hline Cibacron Blue & 774 & $30-50$ & DNA & & {$[171]$} \\
\hline Coenzyme A & 768 & 1 & RNA & 70 & [172] \\
\hline
\end{tabular}

${ }^{1}$ Length of core region of aptamer.

diabetic nephropathy [176]. Besides the above-mentioned aptamers, nucleotidedrugs based on the antisense-RNA technology are available for the treatment of cytomegalovirus retinitis and the reduction of cholesterol (Formivirsen/Vitracene 
and Mipomersen/Kynamro, Isis Pharmaceuticals, Carlsbad, USA) [177, 178]. 


\subsection{Scope of This Work}

There is an absolute need for early and readily applicable methods to detect Fusarium-infected grain and to distinguish between relevant and harmless species. As legal maximum-levels for DON exist, new tools for a fast and reliable detection and quantification of DON have to be developed. This thesis aims at the investigation of Fusarium and deoxynivalenol using new technology for the potential development of rapid tests. The thesis is subdivided into two parts.

1. Fusarium recognition by an electronic nose. Electronic noses have been applied in different fields facilitating the identification and classification of samples, as they provide measurements which are non-destructive, simple, fast, on-line and portable. In this work, an electronic nose should be used to discriminate wheat grain, contaminated by the most widely distributed Fusarium species, namely F. graminearum and F. culmorum and distinguish it from other Fusaria as well as from uninfected grain in the post-harvest field. Furthermore, MVOCs emitted by Fusaria fungi are to be recorded and analysed.

2. Development of aptamers binding deoxynivalenol. Although electronic noses may be capable of detecting DON-producing Fusarium spp., these assays cannot provide a complete quantitative estimation of DON levels. Therefore, a novel DNA-ligand system should be used to select aptamers against deoxynivalenol, with the intent of developing highly stable recognition elements for biosensors in the future. 


\section{Chapter 2}

\section{Materials and Methods}

Grain samples, volatile analysis as well as the Enose measurements are related to Eifler et al. [42].

\subsection{Samples}

\subsubsection{Grain Samples}

Grains from winter soft wheat (Triticum aestivum L., cv. Isengrain, harvest season 2009, Germany) were used. The seeds were water-saturated for 24 hours to ensure rehydration and then autoclaved twice for $15 \mathrm{~min}$ at $121^{\circ} \mathrm{C}$. For each sample, $100 \mathrm{~g}$ sterilised kernels were inoculated with ten $0.5 \mathrm{x} 0.5 \mathrm{~cm}^{2}$ slices of fungal mycelia derived from cultures grown on potato dextrose agar (PDA). Incubation was carried out for $5 \mathrm{~d}, 10 \mathrm{~d}$ and $15 \mathrm{~d}$ at a relative humidity of $70 \%$ and at $27^{\circ} \mathrm{C}$. Infected samples were dried to $13 \%$ moisture content and stored at $4{ }^{\circ} \mathrm{C}$ to block further fungal growing. The used fungus species were Fusarium graminearum, Fusarium culmorum, Fusarium cerealis and Fusarium redolens. Sterilised, non-infected kernels, autoclaved and incubated over 0, 5, 10 or 15 days as well as an completely untreated probe served as controls (cf. Figure 2.1). The number of samples was chosen in respect of the sample variability artificially induced by inoculating microorganisms in homogeneous grain samples. As a consequence, the influence of the natural variability among grains, due to variety variability and crop production was not considered. 

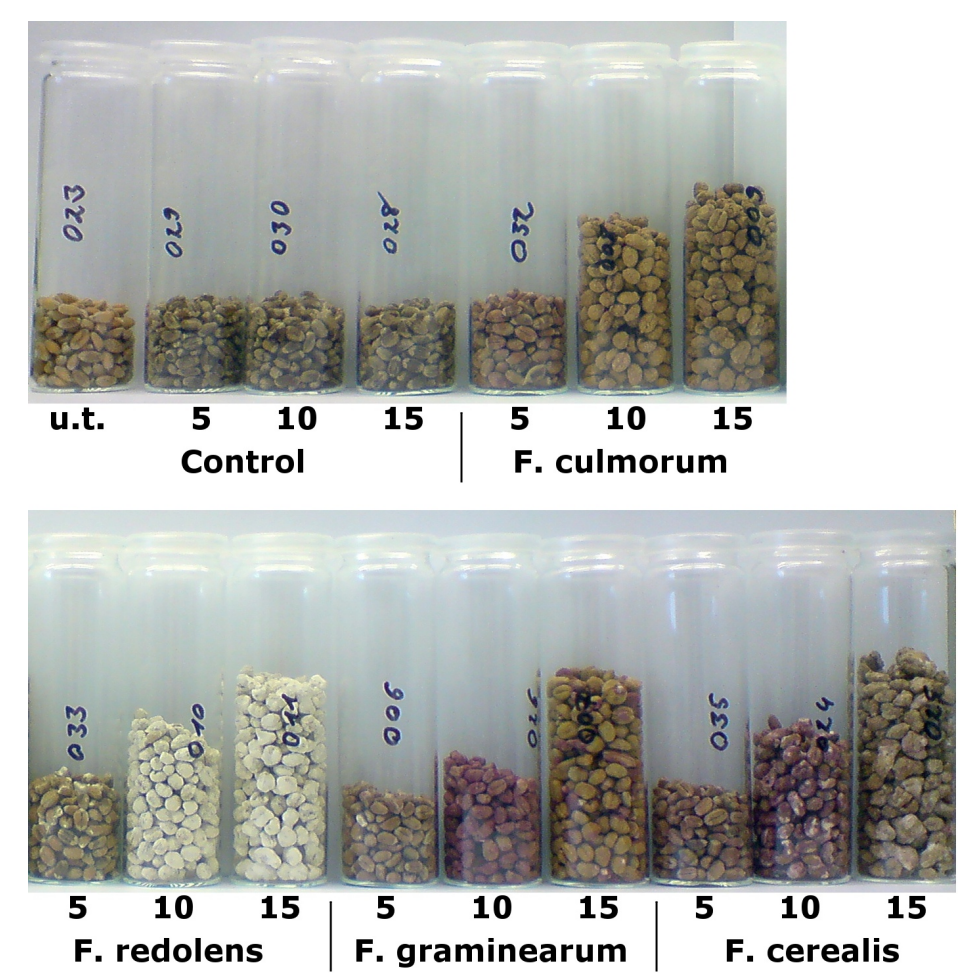

Figure 2.1: Sample preparation of F. culmorum, F. redolens, F. graminearum and $F$. cerealis with different incubation times. Numbers indicate days of incubation. u.t. = untreated control

\subsubsection{Oligonucleotides}

All oligonucleotides were purchased either from Life Technologies Cooperation (Carlsbad, CA, USA) or IBA GmbH (Göttingen, Germany). The initial singlestranded DNA pool containing 40 randomised positions (cf. Table 2.1), was flanked by two constant regions for primer attachment and was ordered as "manual mixing" to guarantee an equal distribution of nucleotides in the random region. A fluorescent label 6-HEX (6-carboxy-2,4,4,5,7,7 -hexachlorofluorescein succinimidyl ester) was attached to the 5' position of the library sequences. The theoretical pool complexity can be estimated to $4^{40}=1.2 \times 10^{24}$ different molecules. For PCR amplification a HEX-labelled forward and a phosphorylated reverse primer were used. Capture oligonucleotides consisted of a complementary region of seven to twenty bases of the 5' constant region of the library (see capDNAs, Table 2.1) 
and an additional biotin modification at the 3' end. All oligonucleotides were HPLC-purified.

Table 2.1: Oligonucleotides used for column-SELEX. Modified after [143].

\begin{tabular}{|c|c|}
\hline Name & Sequence $\left(5^{\prime}-3^{\prime}\right)$ \\
\hline \multicolumn{2}{|l|}{ Library (78-mer) } \\
\hline \multicolumn{2}{|c|}{ 6-HEX-GTCACTGTCTTCATAGGTTG- $N_{40}$-GAATCAGTGAGACATCCC } \\
\hline \multicolumn{2}{|c|}{ Primers } \\
\hline Forward (20-mer) & GTCACTGTCTTCATAGGTTG \\
\hline Forward HEX5'(20-mer) & 6-HEX-GTCACTGTCTTCATAGGTTG \\
\hline Reverse (18-mer) & GGGATGTCTCACTGATTC \\
\hline Reverse Phos (18-mer) & PHOS-GGGATGTCTCACTGATTC \\
\hline \multicolumn{2}{|l|}{ Capture DNAs } \\
\hline capDNA7.1 (8-mer) & CAGTGACT-Biotin \\
\hline capDNA10.1 (11-mer) & GACAGTGACT-Biotin \\
\hline capDNA12.1 (13-mer) & GAAGACAGTGACT-Biotin \\
\hline capDNA14.1 (15-mer) & ATGAAGACAGTGACT-Biotin \\
\hline capDNA16.1 (17-mer) & CTATGAAGACAGTGACT-Biotin \\
\hline capDNA20.1 (21-mer) & CAACCTATGAAGACAGTGACT-Biotin \\
\hline
\end{tabular}

\subsection{Volatile Analysis}

\subsubsection{Headspace Generation}

For both GC/MS and Enose measurements, grain samples (3g) were enclosed in Teflon-sealed vials. Prior to the measurements, the samples were kept in a thermal bath for $30 \mathrm{~min}$ at constant temperature. The Enose experiments were carried out at a sample temperature of $30^{\circ} \mathrm{C}$. GC/MS analyses were done at $30^{\circ} \mathrm{C}$ and $70^{\circ} \mathrm{C}$. In addition, an empty vial was added as a reference air source.

\subsubsection{Gas Chromatographic Analysis}

GC/MS analysis were performed using a gas chromatograph (QP2010, Shimadzu, Japan) connected to a mass spectrometer. An EQUITY-5 capillary column (30 m x $0.25 \mathrm{~mm}$ ID; $0.25 \mu \mathrm{m}$ thick film) was used in splitless mode with a programmed temperature time course (starting at $40^{\circ} \mathrm{C}$ and increasing, up to $250^{\circ} \mathrm{C}$, 


\section{MATERIALS AND METHODS}

at a rate of $10^{\circ} \mathrm{C} \mathrm{min}^{-1}$ and beyond at a rate of $20^{\circ} \mathrm{C} \mathrm{min}^{-1}$, up to $300{ }^{\circ} \mathrm{C}$, followed by a hold time of $2 \mathrm{~min})$. Helium $(\mathrm{p}=14.5 \mathrm{kPa}$ ) was used as carrier gas

with a total flow of $5.4 \mathrm{ml} \mathrm{min}^{-1}$ and a column flow of $0.59 \mathrm{ml} \mathrm{min}^{-1}$. The mass spectrometer worked at an ionisation energy of $70 \mathrm{eV}$ and a mass range from 40 to $300 \mathrm{~m} / \mathrm{z}$. Headspace collection was performed by solid phase microextraction (SPME) with the carboxen/polydimethylsiloxane (CAR-PDMS, $75 \mu \mathrm{m}$ ) fibre (Supelco). After exposure of the fibre to the pre-heated headspace of the grains for $30 \mathrm{~min}$, its contents were injected into the $\mathrm{GC}$ for $1 \mathrm{~min}$ at an injection temperature of $250^{\circ} \mathrm{C}$. Compound identification was done using the NIST library.

\subsubsection{Electronic Nose Analysis}

The core of the electronic nose consisted of an array of eight quartz microbalances, each being a quartz crystal resonator with mass-dependent eigenfrequency f. Slight mass changes $(\Delta m)$ of the quartz surface result in frequency changes $(\Delta f)$ of the electrical output signal of the oscillator circuit. The Enose consisted of QMBs with a fundamental frequency of $20 \mathrm{MHz}$ and a mass sensitivity in the order of a few nanograms. They were coated with layers of metalloporphyrins. Regarding their sensing properties metalloporphyrins host several interaction mechanisms from weak and non-selective Van-der-Waals forces to the more energetic and specific coordination of the central metal atom. The balance between these forces can be controlled by the nature of the porphyrins' peripheral group and the metal atom, so that metalloporphyrins with different sensitivities for volatile compounds can be obtained [179] and assembled to sensor arrays for electronic noses [119].

For the experiments, the grain samples were closed in a sealed vial with an inlet and an outlet. Vials were kept at constant temperature to allow for a stable headspace composition. The headspace was extracted by a flow of ambient air, filtered through a $\mathrm{CaCO}_{3}$ bed. The flow was maintained constant at $7.5 \mathrm{ml} \mathrm{min}^{-1}$ by a peristaltic pump of the electronic nose. The filtered ambient air was also used to clean the sensors and to establish the reference signal. Sensor signals were calculated as the signal frequency shift, $\Delta f=f_{s}-f_{a}$, with $f_{s}$ and $f_{a}$ being obtained from the sample headspace and filtered ambient air. Sensors were 
exposed to a sample for $60 \mathrm{~s}$, followed by a 5 min cleaning and regeneration phase with reference air. All measurements were repeated three times.

The QMB frequency differences, $\Delta f$, between the steady-state reference (air) and the recording phase was used as feature vector. The classification of the different samples was based on a discriminant analysis. Here, the partial least squares discriminant analysis (PLS-DA) was used, which is an algorithm originally developed for quantitative regression [180]. Before the application of PLS-DA, Enose data were properly auto-scaled (zero mean, unitary variance). Discrimination models have been cross-validated by the leave-one-out method in order to estimate the classification performance.

\subsection{Fluorescence Measurements and Fluorophores}

Fluorescence measurements were mainly done with a multilabel plate reader (VictorX2, Perkin Elmer, Waltham, MA) in 96-well plates (NUNC Black, Thermo Scientific) in $100 \mu \mathrm{l}$ per sample. The temperature was kept constant at $25^{\circ} \mathrm{C}$ during measurement. Each measurement was repeated 10 times and the mean value was calculated. A blank probe was always included in a measurement cycle and blank correction was performed for every sample. The aperture was set to 'small' with a measurement time of $1 \mathrm{~s}$. The cw-lamp was driven with stabilised energy at a parameter setting of 15048 .

6-HEX (6-carboxy-2, 4,4 ,5,7,7 -hexachlorofluorescein succinimidyl ester) was chosen as a fluorophore label with respect to the spectrum of SybrGreen I, which should be monitored during PCR. Thereto, the filter set ET530/20 and ET575/50 (Chroma Technology) was installed (cf. Figure 2.2 and 2.3) to cover the maximum excitation and emission spectra. 

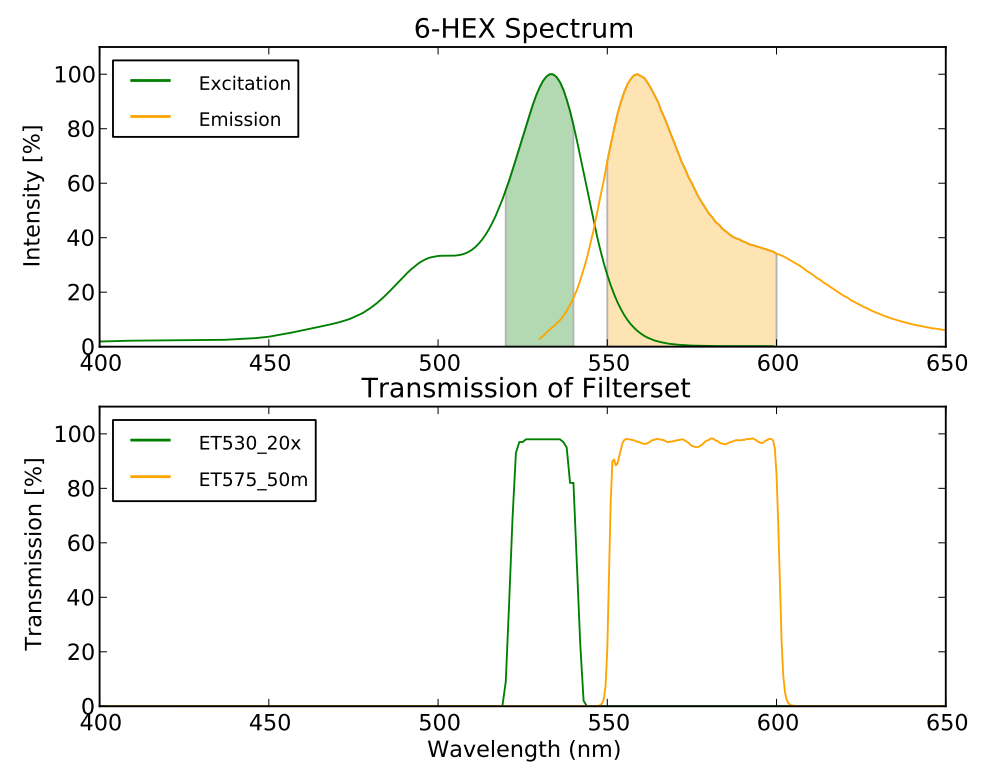

Figure 2.2: Excitation and emission spectrum of 6-HEX and the transmission of the installed filter set (ET530/20 and ET575/50). Excitation maximum: $533 \mathrm{~nm}$; Emission maximum: $559 \mathrm{~nm}[181,182]$.

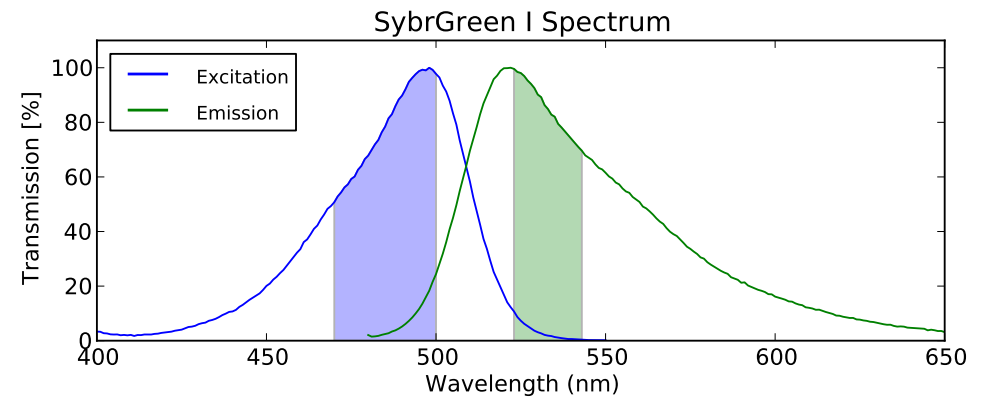

Figure 2.3: Excitation and emission spectrum of SybrGreen I and the coverage of the real-time PCR machine (highlighted regions). Excitation maximum: $489 \mathrm{~nm}$; Emission maximum: $517 \mathrm{~nm}[182,183]$. 


\subsection{Aptamer Generation}

\subsubsection{Optimisation of Capture Oligonucleotide}

As a first step of aptamer selection, the optimal complementary length of the capture oligonucleotide was determined by annealing the aptamer pool to differently sized biotinylated capture oligonucleotides. According to Rajendran and Ellington [143], 50 pmol of the initial library were mixed with 100 pmol of each of the different capture DNAs of 7, 10, 12, 13, 14, 16 and 20 complementary bases in $20 \mu \mathrm{l}$ of DON-Selection-Buffer (DSB, $50 \mathrm{~mm}$ HEPES, $300 \mathrm{~mm} \mathrm{NaCl}, 0.5 \mathrm{mM} \mathrm{MgCl}_{2}$, $\mathrm{pH} 7.0$ ). The reaction was heated to $90^{\circ} \mathrm{C}$ for $30 \mathrm{~s}$, then stepwise cooled to $45^{\circ} \mathrm{C}$ for $90 \mathrm{~s}$ and eventually to room temperature over $10 \mathrm{~min}$. The mixture was afterwards diluted to $100 \mu \mathrm{l}$ DSB and immobilised on $30 \mu \mathrm{l}$ streptavidin-agarose (Sigma-Aldrich, St Louis, MO, USA), pre-washed with three times $300 \mu \mathrm{l}$ DSB. The DNA-agarose complex was transferred to a column (Bio-Rad, Hercules, CA, USA) and incubated 25 min at room temperature in an overhead incubator. After measuring the fluorescence of the eluate, the column was washed ten times, each with five column volumes DSB. Afterwards, it was washed again for ten times of each five column volumes denaturing buffer ( $7 \mathrm{M}$ Urea, 0.1 M Sodium Citrate, $3 \mathrm{~mm}$ EDTA, pH 5.0). Washing fractions were collected and the amount of DNA was estimated by fluorescence measurements.

\subsubsection{Optimisation of PCR Parameters}

Before the bulk amplifications were done, the used Q5 Hot Start High-Fidelity DNA polymerase (New England Biolabs, Frankfurt am Main, Germany) as well as the primers Forward HEX5' and Reverse Phos (cf. Table 2.1) were tested on SybrGreen I addition and annealing temperature in $50 \mu \mathrm{l}$ reactions. The amplification took place on a MiniOpticon (Bio-Rad, Hercules, CA, USA) cycler with the following temperature time course. $30 \mathrm{~s}$ at $98^{\circ} \mathrm{C}$ initial denaturation, 17 cycles of $10 \mathrm{~s}$ at $98^{\circ} \mathrm{C}, 30 \mathrm{~s}$ at gradient 45 to $61^{\circ} \mathrm{C}, 15 \mathrm{~s}$ at $72^{\circ} \mathrm{C}$ and $1 \mathrm{~min}$ final elongation at $72^{\circ} \mathrm{C}$, according to the Q5 supplier instructions. Every reaction mix contained beside $1 \mu \mathrm{M}$ of the primers each, 1x Q5 reaction buffer (New England Biolabs, Frankfurt am Main, Germany), $0.2 \mathrm{~mm}$ of dNTPs each as well as $1 \mathrm{U}$ of the 
polymerase and 0.2 to $0.5 \times$ SybrGreen I (Sigma-Aldrich, St Louis, MO, USA). A similar experiment was carried out with FastStart Taq DNA Polymerase (Roche, Mannheim, Germany), with the exception of testing the annealing temperature and the $\mathrm{MgCl}_{2}$ concentration. Here the time program was 4 min at $95^{\circ} \mathrm{C}, 17 \mathrm{cy}-$ cles of $30 \mathrm{~s}$ at $95^{\circ} \mathrm{C}, 30 \mathrm{~s}$ at 45 to $61^{\circ} \mathrm{C}, 30 \mathrm{~s}$ at $72^{\circ} \mathrm{C}$ and $1 \mathrm{~min}$ final elongation at $72^{\circ} \mathrm{C}$. The reaction contained beside the primers and dNTPs in the concentration mentioned above, $2.5 \mathrm{U}$ polymerase, 1x PCR reaction buffer (Roche, Mannheim, Germany), 0.3x SybrGreen I (Sigma-Aldrich, St Louis, MO, USA) and 1.5 to $3.0 \mathrm{mM} \mathrm{MgCl}_{2}$. In both experiments, the intended ssDNA library was used as template in a concentration of $0.2 \mathrm{nM}$.

\subsubsection{Optimisation of Strand Separation}

The strand separation was done according to Avci-Adali et al. [184]. The phosphorylated reverse strand was thereby digested with Lambda Exonuclease (New England Biolabs, Frankfurt am Main, Germany). For the test, a large bulk PCR was carried out as described above, but with $75 \mu \mathrm{l}$ reactions for 15 cycles. Four of these reactions were pooled and cleaned with a PCR-purification Kit (Qiagen, Germantown, MD) and then digested with $6 \mathrm{U} \mathrm{\mu g}^{-1}$ dsDNA in reaction buffer at different digestion times. The reactions were stopped by incubating for $10 \mathrm{~min}$ at $75^{\circ} \mathrm{C}$ at the desired time and displayed within a $3 \%$ agarose (Agarose II, Amresco, Solon, USA) gel.

\subsubsection{Implementation of Column SELEX}

The selection routine was chosen according to Rajendran and Ellington [145], and includes the development of aptamer beacons. SELEX was initiated by coupling $1.1 \mathrm{nmol}$ of the initial, random DNA pool with a two-fold excess of the desired capture oligonucleotide capDNA12.1 in $22 \mu \mathrm{l}$ of DSB. The used amount was chosen due to practical reasons and corresponded to a diversity of $6.6 \times 10^{14}$ different molecules, which is about $5 \times 10^{-8} \%$ of the complete pool. After a heat treatment of $94^{\circ} \mathrm{C}$ for $30 \mathrm{~s}$, the annealing reaction was cooled down to $45^{\circ} \mathrm{C}$ for $90 \mathrm{~s}$ and then to room temperature. The annealed pool was diluted to $600 \mu \mathrm{l} \mathrm{DSB}$ and subsequently transferred to a streptavidin-agarose (Sigma-Aldrich, St Louis, 
MO, USA) filled column (Bio-Rad, Hercules, CA, USA). The column was equilibrated three times with $800 \mu \mathrm{l}$ DSB. Incubation of the pool-capDNA complex was carried out for $45 \mathrm{~min}$ at room temperature within an overhead incubator. The column was then drained and washed five times with $800 \mu \mathrm{l}$ DSB, 3 min each. The unbound DNA in the eluate as well as the washing-fractions were measured by fluorescence. DON aptamers were eluted with DSB containing $2 \mathrm{mM}$ DON for $30 \mathrm{~min}$ followed by three DSB washes à $400 \mu \mathrm{l}$. All fractions were collected, pooled and precipitated with ethanol. $10 \%$ of the collection was stored for potential recovery. The residue was amplified and purified to obtain the new pool for the subsequent rounds of selection (cf. Steps 0 to 10 Figure 2.4). The amount of DNA and the column volume were varied due to an increase of stringency (cf. Table 2.2). The DON elution volume was adapted from $600 \mu l$ (round 1) to $200 \mathrm{\mu l}$ and then kept constant. To remove unbound ssDNA and unwanted binders from the column, the washing volume was increased progressively and negative selections were additionally introduced in round four and nine prior to elution (see Table 2.3). The negative selections were comprised of a long DSB incubation of $30 \mathrm{~min}$ (N1), followed by a short wash of $3 \mathrm{~min}$ (N2), lacking DON.

After each round of selection, bulk amplification (cf. Step 6 Figure 2.4) was done on a real-time cycler (MiniOpticon, Bio-Rad, Hercules, CA, USA) in 30 parallel reactions à $75 \mu \mathrm{l}$. Each contained 1x Q5 reaction buffer (New England Biolabs, Frankfurt am Main, Germany), 0.2 mm dNTPs each, 1 1 M HEX-labelled forward and a phosphorylated reverse primer (cf. Table 2.1) and 1.5 U of Q5 Hot Start High-Fidelity DNA polymerase (New England Biolabs, Frankfurt am Main, Germany). Additionally, 6 out of 30 reactions contained 0.3x SybrGreen I (SigmaAldrich, St Louis, MO, USA) for online monitoring. Amplification was stopped when the amount of DNA reached 60 to $90 \%$ of its maximum value, which was generally after 7 to 10 cycles the case. The amplification conditions were $30 \mathrm{~s}$ at $98^{\circ} \mathrm{C} ; 7$ to 9 cycles of $10 \mathrm{~s}$ at $98^{\circ} \mathrm{C}, 30 \mathrm{~s}$ at 57 to $61^{\circ} \mathrm{C}, 15 \mathrm{~s}$ at $72^{\circ} \mathrm{C}$ and $2 \mathrm{~min}$ final elongation at $72{ }^{\circ} \mathrm{C}$ after the last cycle. A negative control was always included to avoid cross-contamination. Gel electrophoresis was carried out to control the success of amplification. Starting from round 11 the amplification reactions were changed as follows. Q5 DNA polymerase and 1x Q5 reaction buffer was replaced by 3.75 U FastStart Taq DNA Polymerase (Roche, Mannheim, Germany) and 
Table 2.2: Input ssDNA, volumes and incubation times used for different rounds of selection.

\begin{tabular}{crrccc}
\hline Round & \multicolumn{2}{c}{ ssDNA } & Column Volume & \multicolumn{2}{c}{ Incubation } \\
& $(\mathrm{pmol})$ & $(\mu \mathrm{\mu})$ & $(\mu \mathrm{l})$ & $(\mathrm{\mu l})$ & $(\mathrm{min})$ \\
\hline 1 & 1105 & 1.84 & 500 & 600 & 45 \\
2 & 74 & 0.37 & 100 & 200 & 45 \\
3 & 120 & 0.56 & 100 & 214 & 45 \\
4 & 94 & 0.45 & 100 & 209 & 45 \\
5 & 91 & 0.46 & 100 & 200 & 45 \\
\hline 6 & 100 & 0.50 & 100 & 200 & 45 \\
7 & 112 & 0.56 & 100 & 200 & 45 \\
8 & 89 & 0.45 & 100 & 200 & 45 \\
9 & 100 & 0.50 & 100 & 200 & 45 \\
10 & 108 & 0.54 & 100 & 200 & 45 \\
\hline 11 & 100 & 0.50 & 100 & 200 & 45 \\
12 & 60 & 0.40 & 60 & 200 & 45 \\
13 & 63 & 0.42 & 60 & 200 & 45 \\
14 & 60 & 0.40 & 60 & 200 & 45 \\
15 & 40 & 0.40 & 40 & 200 & 45 \\
\hline 16 & 40 & 0.40 & 40 & 200 & 45 \\
\hline
\end{tabular}

1x PCR reaction buffer (Roche, Mannheim, Germany) with $2.5 \mathrm{mM} \mathrm{MgCl}_{2}$. The temperature sequence was adapted to $4 \mathrm{~min}$ at $95^{\circ} \mathrm{C} ; 7$ to 9 cycles of $30 \mathrm{~s}$ at $95^{\circ} \mathrm{C}$, $30 \mathrm{~s}$ at 57 to $61^{\circ} \mathrm{C}, 30 \mathrm{~s}$ at $72^{\circ} \mathrm{C}$ and $2 \mathrm{~min}$ final elongation at $72^{\circ} \mathrm{C}$. After PCR all reactions were pooled, ethanol precipitated, filter purified and concentrated (PCR-purification Kit, Qiagen, Germantown, MD).

According to Avci-Adali et al. [184], strand separation was done by digesting the phosphorylated reverse strand with Lambda Exonuclease (New England Biolabs, Frankfurt am Main, Germany). $45 \mu \mathrm{l}$ of dsDNA, which was resuspended in water, was mixed with $5 \mu \mathrm{l}$ of $10 \mathrm{x}$ reaction-buffer ( $67 \mathrm{~mm}$ Glycine-KOH, $2.5 \mathrm{mM} \mathrm{MgCl}_{2}, 50 \mathrm{mg} \mathrm{ml}^{-1} \mathrm{BSA}, \mathrm{pH}$ 9.4) allocated by the exonuclease supplier.

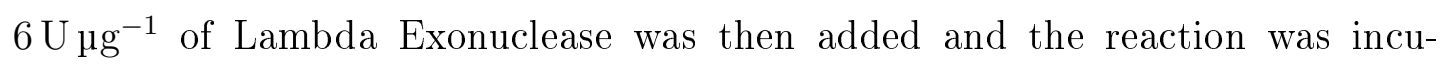
bated at $37^{\circ} \mathrm{C}$ at $300 \mathrm{rpm}$ for $90 \mathrm{~min}$ in a thermomixer (Eppendorf, Hamburg, Germany). To stop the reaction, the mixture was either immediately purified by filter concentration (Nucleotide Removal Kit, Qiagen, Germantown, MD) or 
Table 2.3: Pre-SELEX washes for different rounds of SELEX. Each wash step was done with DSB and included three minute incubation. Negative selections were carried out with DSB for 30 min and 3 min, respectively.

\begin{tabular}{cccccc}
\hline Round & $\begin{array}{c}\text { Total } \\
\text { Volume } \\
\text { (Col.Vol. })\end{array}$ & $\begin{array}{c}\text { Step } \\
\text { Volume } \\
(\mu \mathrm{l})\end{array}$ & $\begin{array}{c}\text { Step } \\
\text { Repetition } \\
\text { (x times) }\end{array}$ & $\begin{array}{c}\text { Negative Selection } \\
\text { N1 }\end{array}$ & $\begin{array}{c}\text { N2 }) \\
(\mu \mathrm{l})\end{array}$ \\
\hline 1 & 8 & 800 & 5 & - & - \\
2 & 12 & 240 & 5 & - & - \\
3 & 14 & 280 & 5 & - & - \\
4 & 16 & 320 & 4 & 200 & - \\
5 & 20 & 450 & 4 & 200 & - \\
\hline 6 & 25 & 450 & 5 & 250 & - \\
7 & 35 & 525 & 6 & 350 & - \\
8 & 40 & 507 & 7 & 450 & - \\
9 & 52 & 563 & 8 & 500 & 200 \\
10 & 52 & 563 & 8 & 500 & 200 \\
\hline 11 & 52 & 550 & 8 & 500 & 300 \\
12 & 62 & 380 & 8 & 300 & 380 \\
13 & 72 & 440 & 8 & 360 & 440 \\
14 & 82 & 500 & 8 & 420 & 500 \\
15 & 100 & 409 & 8 & 320 & 409 \\
\hline 16 & 130 & 533 & 8 & 400 & 533 \\
\hline
\end{tabular}




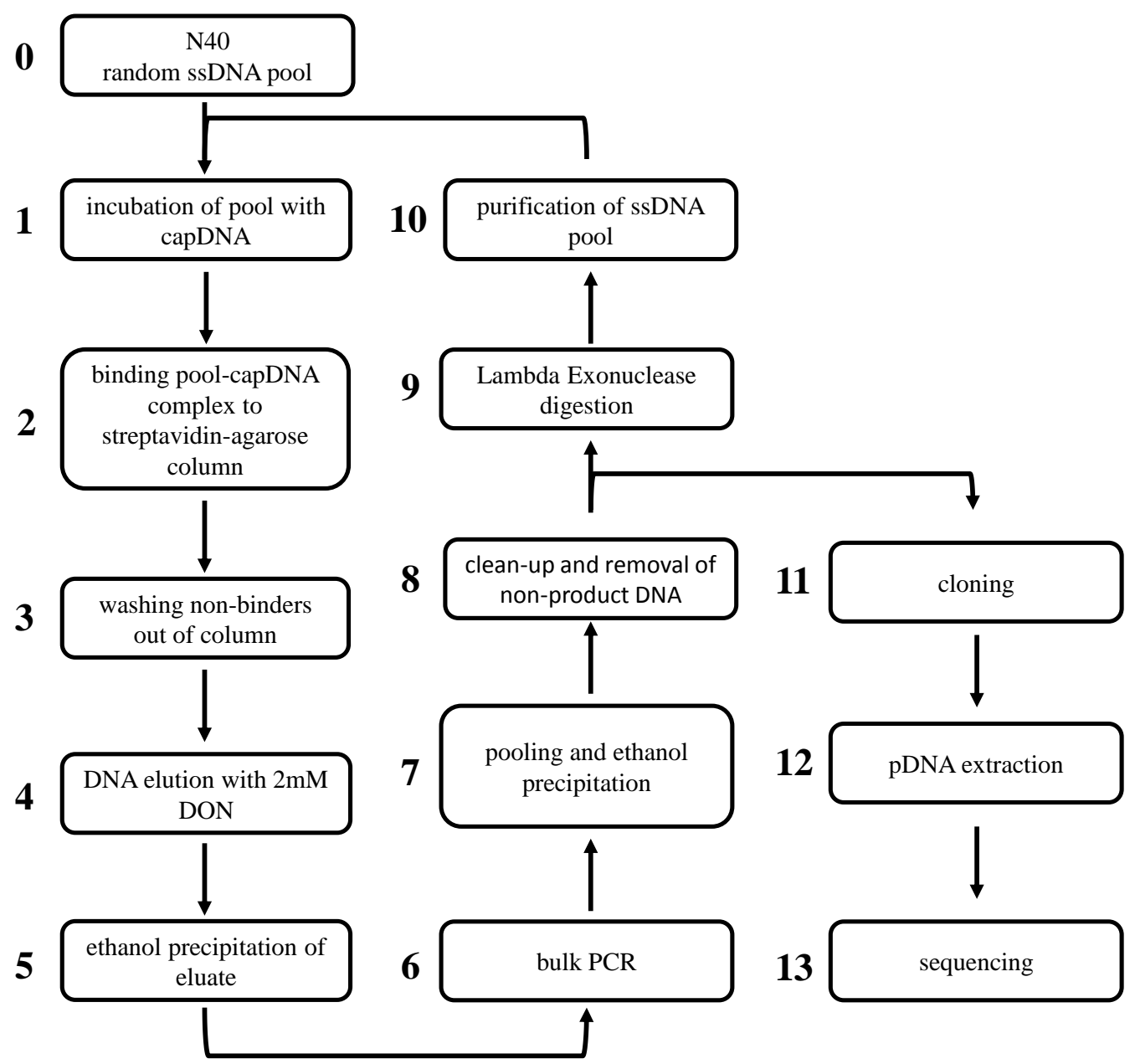

Figure 2.4: DON-aptamer selection scheme. Steps of selection for DON-aptamers and further processing. 
incubated for $10 \mathrm{~min}$ at $75^{\circ} \mathrm{C}$.

\subsubsection{Identifying Individual Aptamers}

The optimal pool from selection was chosen and cloned (Step 11 Figure 2.4), applying the TOP10 TA-Cloning Kit (Invitrogen, Carlsbad, USA) according to the instructions of the supplier. In brief, bulk PCR with unlabelled primers (cf. Table 2.1) were carried out for seven rounds with FastStart Taq DNA Polymerase (Roche, Mannheim, Germany). The Taq DNA polymerase has a non-templatedependent activity to add an additional deoxyadenosine (dA) to the 3' end of the PCR-product, which is used in TA-Cloning for ligation. The resulting dsDNA was then purified (PCR Purification Kit, Qiagen, Germantown, MD) and the amplicon was cloned into a $\mathrm{pCR}^{\mathrm{TM}} 2.1-\mathrm{TOPO}^{\circledR}$ vector. The vector was inserted into TOP10 competent cells of Escherichia Coli by chemical transformation. The transformants were then plated on LB-Medium and the colonies separated. pDNAs were extracted (Step 12 Figure 2.4) by PureLink Quick Plasmid Miniprep Kit (Invitrogen, Carlsbad, USA) and analysed on the existence of the insert by digesting a small volume with EcoR I (Invitrogen, Carlsbad, USA). The inserted aptamer DNA in positive clones were sequenced (Seqlab GmbH, Göttingen) and analysed on their secondary structure using the UNAfold Software by Markham and Zuker [185].

\subsection{Binding Assay}

To test the selected and isolated aptamers on their DON-binding capability, binding assays similar to the selections experiments were performed. 40 pmol of a single, HEX-labelled, aptamer clone (Life Technologies Cooperation, Carlsbad, CA, USA) was annealed with 80 pmol of capDNA 12.1 in $50 \mu l$ DON-selection buffer (DSB). The mixture was then heated up to $94{ }^{\circ} \mathrm{C}$ for $30 \mathrm{~s}$, cooled and kept at $45^{\circ} \mathrm{C}$ for $90 \mathrm{~s}$, and subsequently transferred to room temperature for at least $10 \mathrm{~min}$. The complex was filled up to $200 \mu \mathrm{L}$ DSB and then transferred to $50 \mu \mathrm{l}$ streptavidin-agarose (Sigma-Aldrich, St Louis, MO, USA), previously washed three times with $300 \mu \mathrm{l}$ DSB, respectively. After 45 min incubation, the unbound 
fraction was collected and the column was washed three times with $300 \mu$ DSB. After washing, $200 \mathrm{\mu l}$ DSB containing $2 \mathrm{~mm}$ DON were added and the mixture was again incubated for $30 \mathrm{~min}$ at room temperature prior to eluting the column with two times $500 \mu \mathrm{l}$ DSB. The eluted ssDNA was measured and represented as relative eluted DNA (RED, cf. Equation 2.1). In addition, a negative control was performed similarly with the exception of adding $200 \mu \mathrm{l}$ DSB lacking DON instead of DON-DSB prior to the last incubation step.

$$
\begin{aligned}
R E D & =\frac{F_{e} \cdot V_{e}}{F_{i} \cdot V_{i}-F_{w} \cdot V_{w}} \\
\text { with } & \\
F_{e} & =\text { fluorescence in the eluted fraction } \\
V_{e} & =\text { volume of eluted fraction } \\
F_{i} & =\text { initial fluorescence } \\
V_{i} & =\text { initial incubation volume } \\
F_{w} & =\text { fluorescence of washing fraction } \\
V_{w} & =\text { volume of washing fraction }
\end{aligned}
$$

For the comparison of DON-eluted samples with their negative controls the change in relative eluted DNA $(\triangle \mathrm{RED})$ was calculated according to Equation 2.2, where $R E D_{D O N}$ is the measured relative eluted DNA in the DON-treated sample and $R E D_{c}$ is the relative eluted DNA of the control sample in the absence of DON. Samples with positive $\triangle R E D$ values were repeated three times. $\triangle R E D$ was analysed with a one sample t-test assuming that the null hypothesis is $\mu_{\Delta \mathrm{RED}}=0$ and the alternative is $\mu_{\triangle \mathrm{RED}} \neq 0$.

$$
\triangle R E D=R E D_{D O N}-R E D_{c}
$$




\section{Chapter 3}

\section{Results}

\subsection{Electronic Nose-Based Detection of Fusarium spp. in Wheat Grain}

In a first set of experiments, wheat kernels artificially inoculated with Fusarium were analysed on their volatiles and an electronic nose was used to differentiate between different states of infection [42].

\subsubsection{Volatiles Released by Fusarium}

The analysis of the headspace of Fusarium-infected wheat kernels revealed a complex composition of odours (cf. Table 3.1). Substances such as ethanol, hexanal, hexadecane and 3,7-dimethyl-decane could be identified in all samples. Several other compounds reported to be infection-specific, in particular 2-methyl-1-propanol, 3-methyl-butanol and 1-octen-3-ol were found in almost all species, with the exception of 2-methyl-1-propanol which could not be identified in the F. graminearum probe. The carbonyls were more species specific. While 3 -octanone was only found in the presence of F. graminearum, benzeneacetaldehyde was found in the probes of $F$. cerealis and F. redolens. From the hydrocarbons only p-xylene was not present in control but in all species except $F$. cerealis. Furthermore, F. culmorum was the only species not showing the release of butyrolactone, but evaporated together with F. graminearum 2,2-dimethyl-1-propanol 
benzoate.

Table 3.1: Volatile compounds released by the analysed fungi and an untreated control (sampling temperature, $70^{\circ} \mathrm{C}$ ) [42]. F.cer. $=$ F.cerealis, F. gram. $=$ F.graminearum, F. cul. $=$ F. culmorum, F.red. $=$ F. redolens.

\begin{tabular}{lccccc}
\hline Compound & Control & F. cer. & F. gram. & F. cul. & F. red. \\
\hline Alcohols & & & & & \\
Ethanol & $\mathrm{X}$ & $\mathrm{X}$ & $\mathrm{X}$ & $\mathrm{X}$ & $\mathrm{X}$ \\
2-methyl-1-propanol & & $\mathrm{X}$ & & $\mathrm{X}$ & $\mathrm{X}$ \\
3-methyl-butanol & & $\mathrm{X}$ & $\mathrm{X}$ & $\mathrm{X}$ & $\mathrm{X}$ \\
1-octen-3-ol & & $\mathrm{X}$ & $\mathrm{X}$ & $\mathrm{X}$ & $\mathrm{X}$ \\
Carbonyls & & $\mathrm{X}$ & & & \\
2-methyl-propanal & $\mathrm{X}$ & $\mathrm{X}$ & $\mathrm{X}$ & $\mathrm{X}$ & $\mathrm{X}$ \\
Acetic Acid & & $\mathrm{X}$ & $\mathrm{X}$ & $\mathrm{X}$ & $\mathrm{X}$ \\
3-methyl-butanal & $\mathrm{X}$ & $\mathrm{X}$ & $\mathrm{X}$ & $\mathrm{X}$ & $\mathrm{X}$ \\
Hexanal & & & $\mathrm{X}$ & & \\
3-octanone & & $\mathrm{X}$ & & & $\mathrm{X}$ \\
Benzeneacetaldehyde & & & & & \\
Hydrocarbons & & $\mathrm{X}$ & $\mathrm{X}$ & $\mathrm{X}$ & $\mathrm{X}$ \\
p-xylene & $\mathrm{X}$ & $\mathrm{X}$ & $\mathrm{X}$ & $\mathrm{X}$ & $\mathrm{X}$ \\
Hexadecane & $\mathrm{X}$ & $\mathrm{X}$ & $\mathrm{X}$ & $\mathrm{X}$ & $\mathrm{X}$ \\
3,3,4-trimethyl-hexane & $\mathrm{X}$ & & & & \\
3,7-dimethyl-decane & & $\mathrm{X}$ & $\mathrm{X}$ & & $\mathrm{X}$ \\
Miscellaneous & & $\mathrm{X}$ & $\mathrm{X}$ & \\
$\begin{array}{l}\text { Butyrolactone } \\
\text { 2,2-dimethyl-1-propanol }\end{array}$ & & & & \\
benzoate & & & & & \\
\hline
\end{tabular}

The total amount of volatiles found in the GC/MS analysis clearly increased with increasing sampling temperature. At $70^{\circ} \mathrm{C}$ infected samples emitted approximately 5 -fold more volatiles than at $30^{\circ} \mathrm{C}$, whereas this increase was just $60 \%$ in control samples (cf. Figure 3.1). However, the data obtained from the Enose at $70^{\circ} \mathrm{C}$ were markedly less reproducible leading to worse classifications. Thus, for the Enose experiment a sampling temperature of $30^{\circ} \mathrm{C}$ was chosen. 


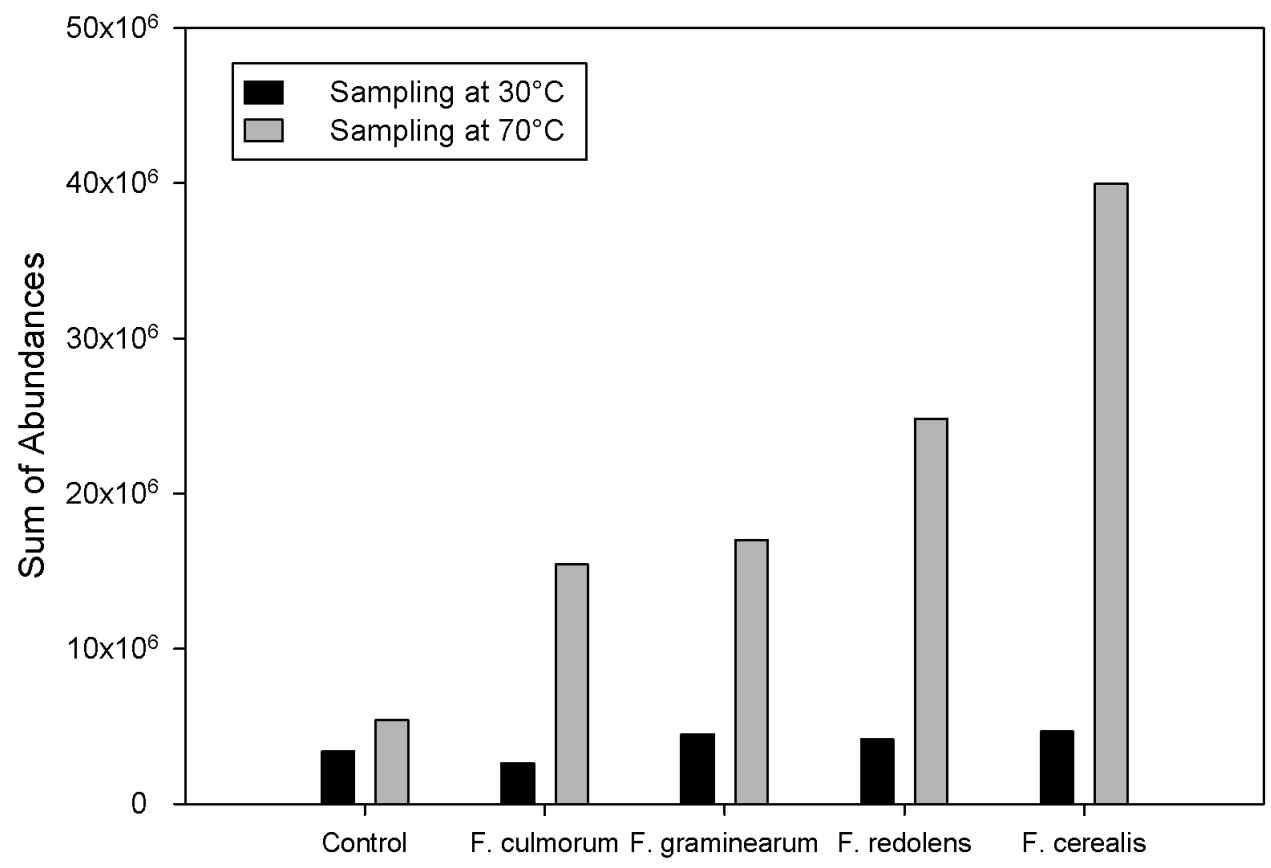

Figure 3.1: Comparison of total volatile abundances of four Fusaria species and controls as obtained from the GC/MS measurements taken at two different sampling temperatures [42].

\subsubsection{Species Identification}

Initially, the capability of the Enose to differentiate between the Fusarium strains was investigated. The PLS-DA model used for this discrimination in the infected samples covered five latent variables, the first two of which are shown in Figure 3.2. The smallest variance within a group occurred in the samples of $F$. cerealis. Samples of this group were overlapping with the highly contaminated samples of $F$. redolens and $F$. culmorum. The widest distribution was found in the samples of $F$. graminearum showing a partial overlap with $F$. culmorum and $F$. cerealis. It can be noticed that the gas chromatographic profiles from F. redolens and F. culmorum are similar. However, there was a large interclass variance detectable.

The model has a satisfactory capability of distinguishing different classes, as 


\section{RESULTS}

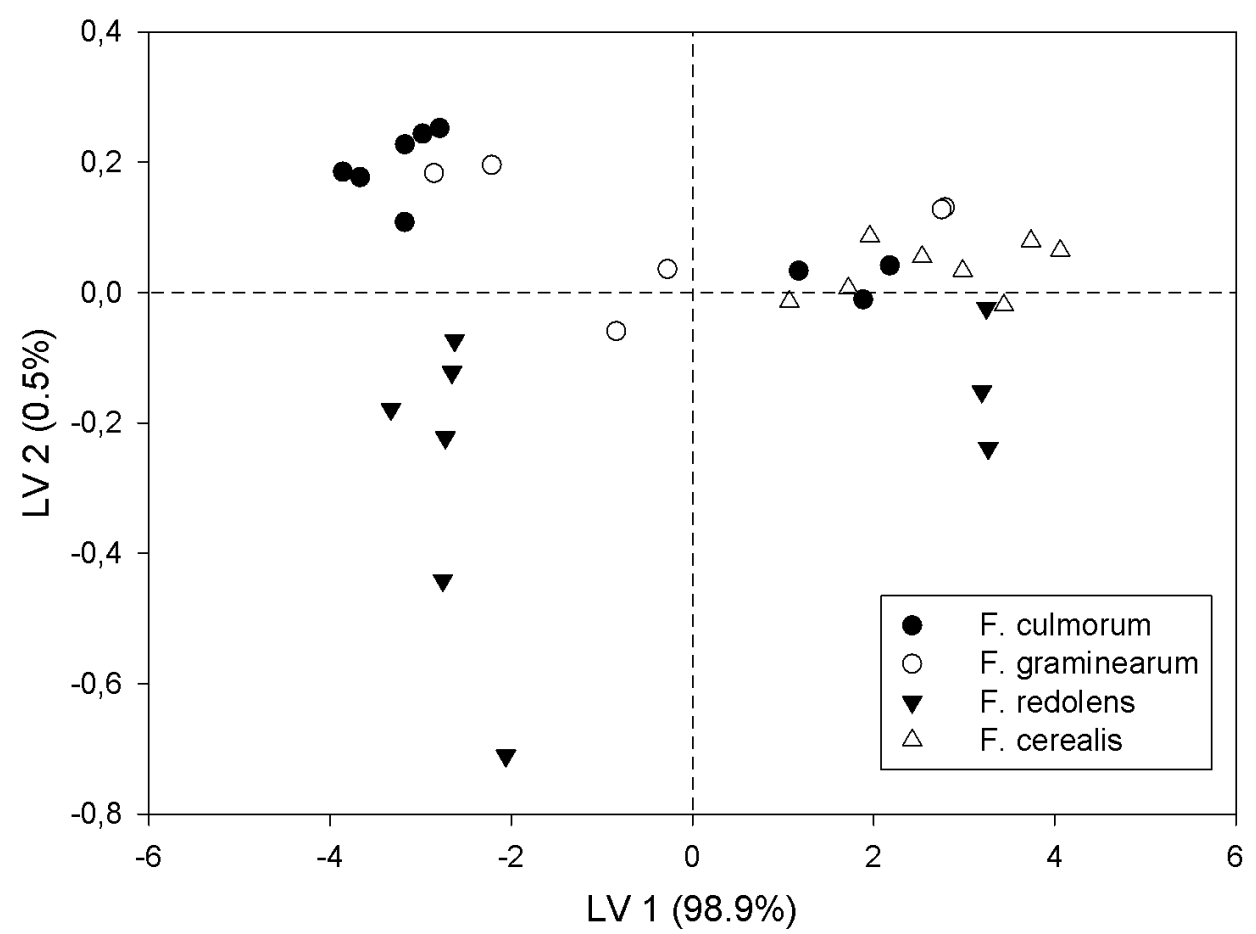

Figure 3.2: Scores plot of the first two latent variables, showing groups of $F$. culmorum, F. graminearum, F. redolens and F. cerealis [42].

confirmed by the confusion matrix (cf. Table 3.2). In the cross-validation F. culmorum and F. cerealis were perfectly recognised (100\% correct). The classification rates of $F$. graminearum and F. redolens were $83 \%$ and $89 \%$, respectively. The correct classification rate across all fungi was $94 \%$.

Table 3.2: Confusion matrix of true vs. estimated values of species classification [42]. Classification was done by PLS-DA.

\begin{tabular}{lcccc}
\hline True Value & Estimated Value & & & \\
\hline & F.culmorum & F.graminearum & F. redolens & F. cerealis \\
\hline F. culmorum & 9 & 0 & 0 & 0 \\
F. graminearum & 0 & 5 & 0 & 1 \\
F. redolens & 0 & 0 & 8 & 1 \\
F. cerealis & 0 & 0 & 0 & 8 \\
\hline
\end{tabular}




\subsubsection{Classification of Infection Level}

Classifying the level of infection properly turned out to be slightly more difficult due to a high interspecies variability and the need for many data samples at the various concentrations. Thus, a binary classification with three latent variables in total was done (cf. Figure 3.3). For that purpose samples with an incubation time of 15 days were classified as highly infected, while samples having been incubated over 5 or 10 days were merged and classified as low-infected. This led to a higher variability in the low level probes. Nevertheless, an obvious distinction could be detected. Classification of the infection level (cf. Table 3.3) showed that $91 \%$ of the samples were correctly classified. However, $18 \%$ of highly infected samples were classified as low-infected, all of them being F. graminearum samples.

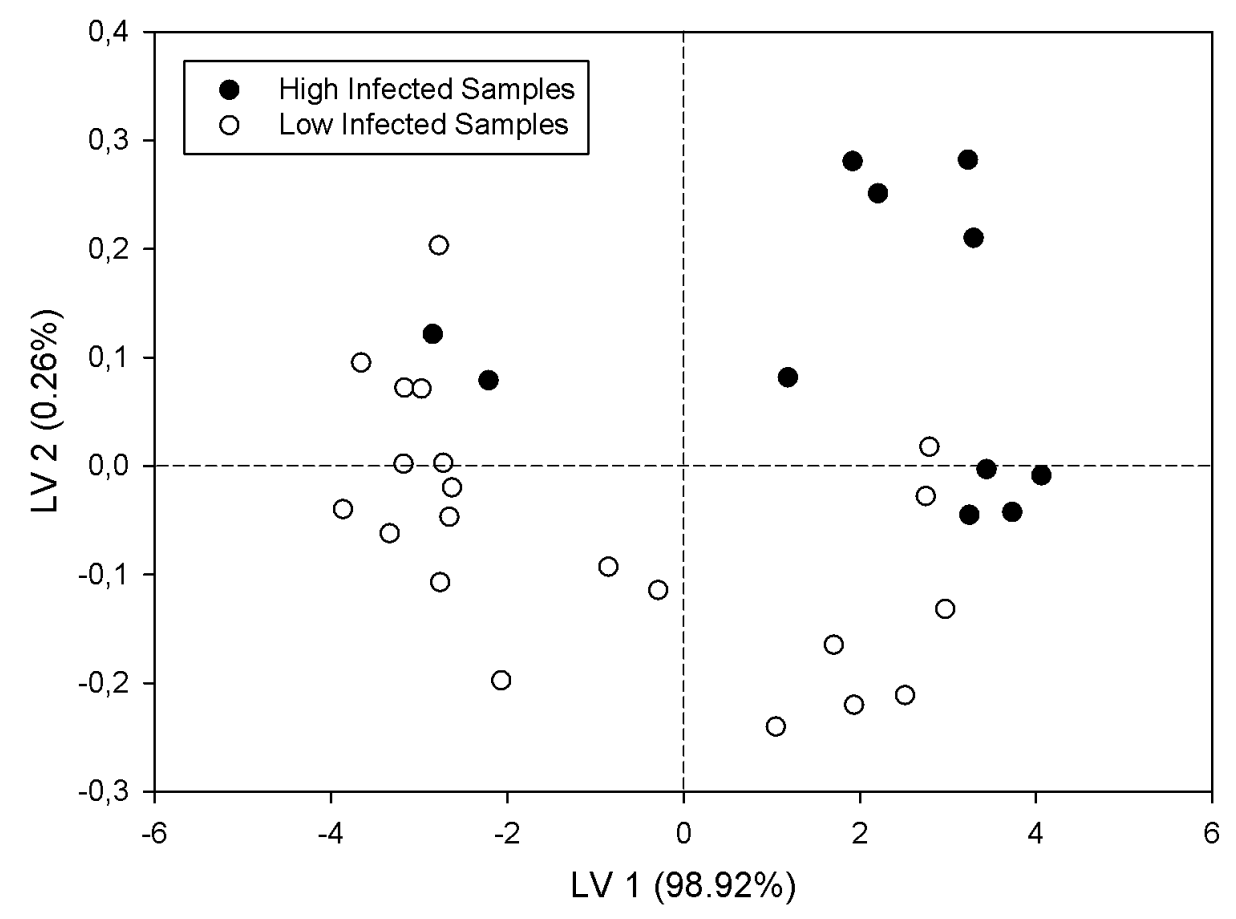

Figure 3.3: Discrimination between high (15 days incubation) and low infected (5 to 10 days incubation) samples of Fusarium [42]. The diagram shows the scores of the first two out of 3 latent variables. 
Table 3.3: Confusion matrix of true vs. estimated values of binary classification of infection levels. Samples with incubation times of 5 to 10 days were classified as low, samples with 15 days as high [42]. Classification was done by PLS-DA.

\begin{tabular}{lcc}
\hline True Value & Estimated Value & \\
\hline & Low & High \\
\hline Low & 20 & 1 \\
High & 2 & 9 \\
\hline
\end{tabular}

\subsubsection{Fungal Infection vs. Control}

For a qualitative analysis, all measurements were split into two groups (infected vs. control). Figure 3.4 shows average sensor responses of infected vs. control samples or ambient air. For the infected samples, the responses of all sensors were on average $16 \mathrm{~Hz}$ higher than for the controls. Ambient air gave very low responses. The signals were lower than $50 \%$ as compared to grain measurements.

Furthermore, a PLS-DA model was performed on these data with a total of four latent variables. The scores plot of the first two variables (cf. Figure 3.5) showed a segregation of infected from control samples, although with a slight overlap. Accordingly, the major portion (91\%) of infected probes are recognised correctly by cross-validation (confusion matrix, Table 3.4). However, eight out of 47 measurements led to a misclassification due to large variances within the groups. Notably, all of the false negatives originated from F. redolens at a high infection level. The discrimination model revealed on average a correct classification of $83 \%$.

Table 3.4: Confusion matrix of true vs. estimated values, summarising the classification results of infection [42]. Estimation was done by PLS-DA classifier.

\begin{tabular}{lcc}
\hline True Value & Estimated Value & \\
\hline & Control & Infected \\
\hline Control & 10 & 5 \\
Infected & 3 & 29 \\
\hline
\end{tabular}




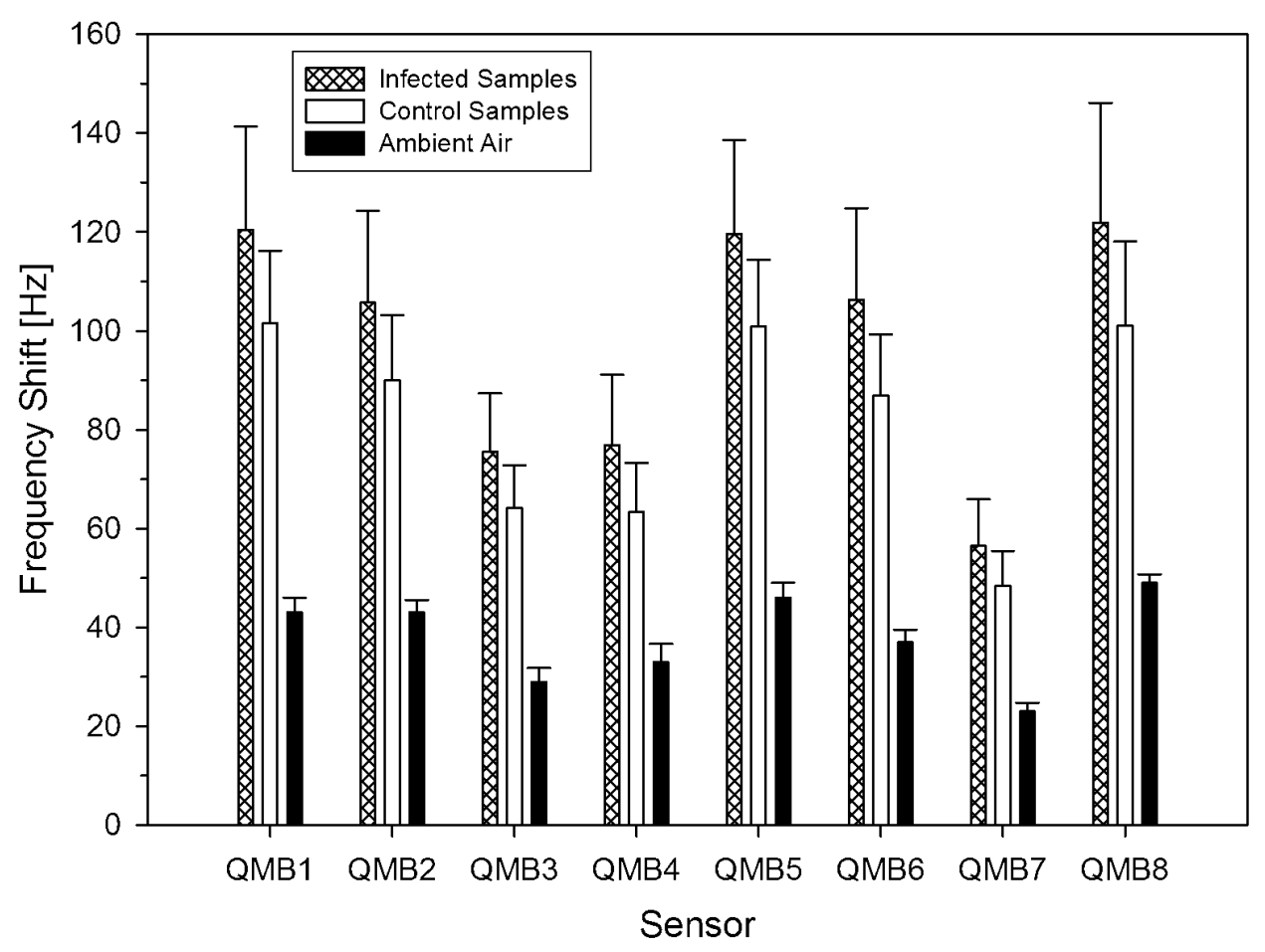

Figure 3.4: Frequency shift of the quartz microbalance sensors during measurements of infected $(n=32)$ and control $(n=15)$ samples, and of ambient air $(n=5)$ [42]. Error bars indicate standard deviations. 


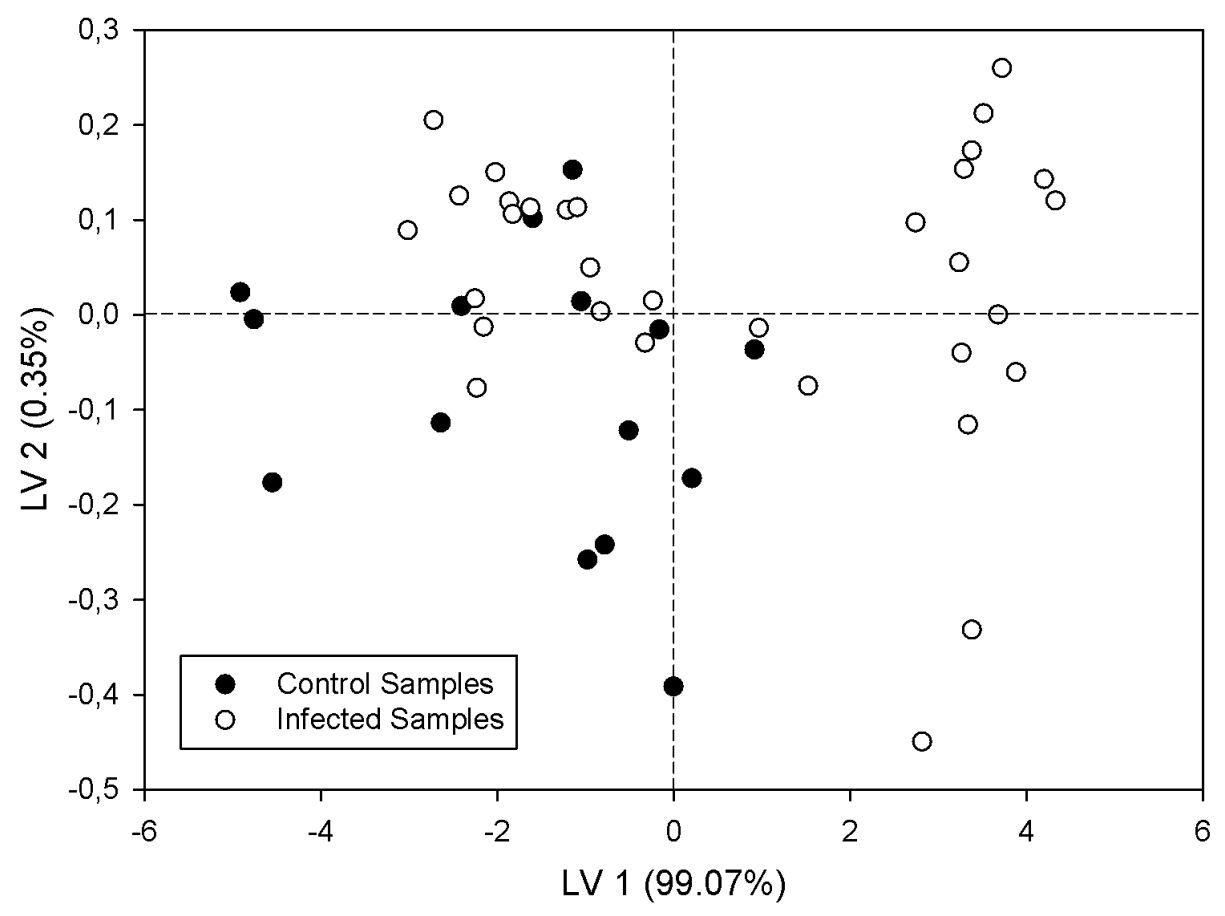

Figure 3.5: Scores plot of the first two latent variables of the PLS-DA model showing groups of infected and non-infected samples [42]. The model covers four latent variables in total. 


\subsection{Selecting Aptamers against Deoxynivalenol}

As deoxynivalenol is the most often occurring mycotoxin due to Fusarium infection, aptamers binding DON were selected in a column-SELEX. Before the experiment, pre-SELEX tests were carried out to optimise the individual steps of selection.

\subsubsection{Influence of Capture Oligonucleotide-Length on Bind- ing Affinity}

The strength of hybridisation and thus the ability of the affinity column to bind and release DNA is dependent on the length of the complementary nucleotide chain. Figure 3.6 shows how the binding of the initial ssDNA pool to the affinity column depends on the different capture oligonucleotide-lengths.

As expected, the smaller the hybridised region the less DNA binds to the column at all and the more DNA will be released during buffer wash. In the case of a length of seven, only $55 \%$ of the initial DNA binds to the column and out of that $33 \%$ were washed out during the first wash of binding buffer. The loss continues steadily with ongoing washes. Eventually, only $32 \%$ of the initial DNA remained on the column after 10 washing steps. In the case of 12 and more complementary bases $90 \%$ and more of the DNA retained on the column in the initial state and the loss during the first wash is with 2 to $6 \%$ almost negligible. Even during further washes the amount of DNA on the column remains constant. In contrast, during the use of denaturing buffer, indicating the disruption of the binding, a large amount of DNA (31 to $45 \%$ of total DNA) can be recovered, if there is enough DNA left on the column (number of $\mathrm{bp}>10$ ) and the bond is not too tight (number of $\mathrm{bp}<20$ ). Since the proportion of binding and release upon disruption is essential for the analyte-dependent selection, the capture oligonucleotide capDNA12.1 was chosen for the further SELEX experiment. 


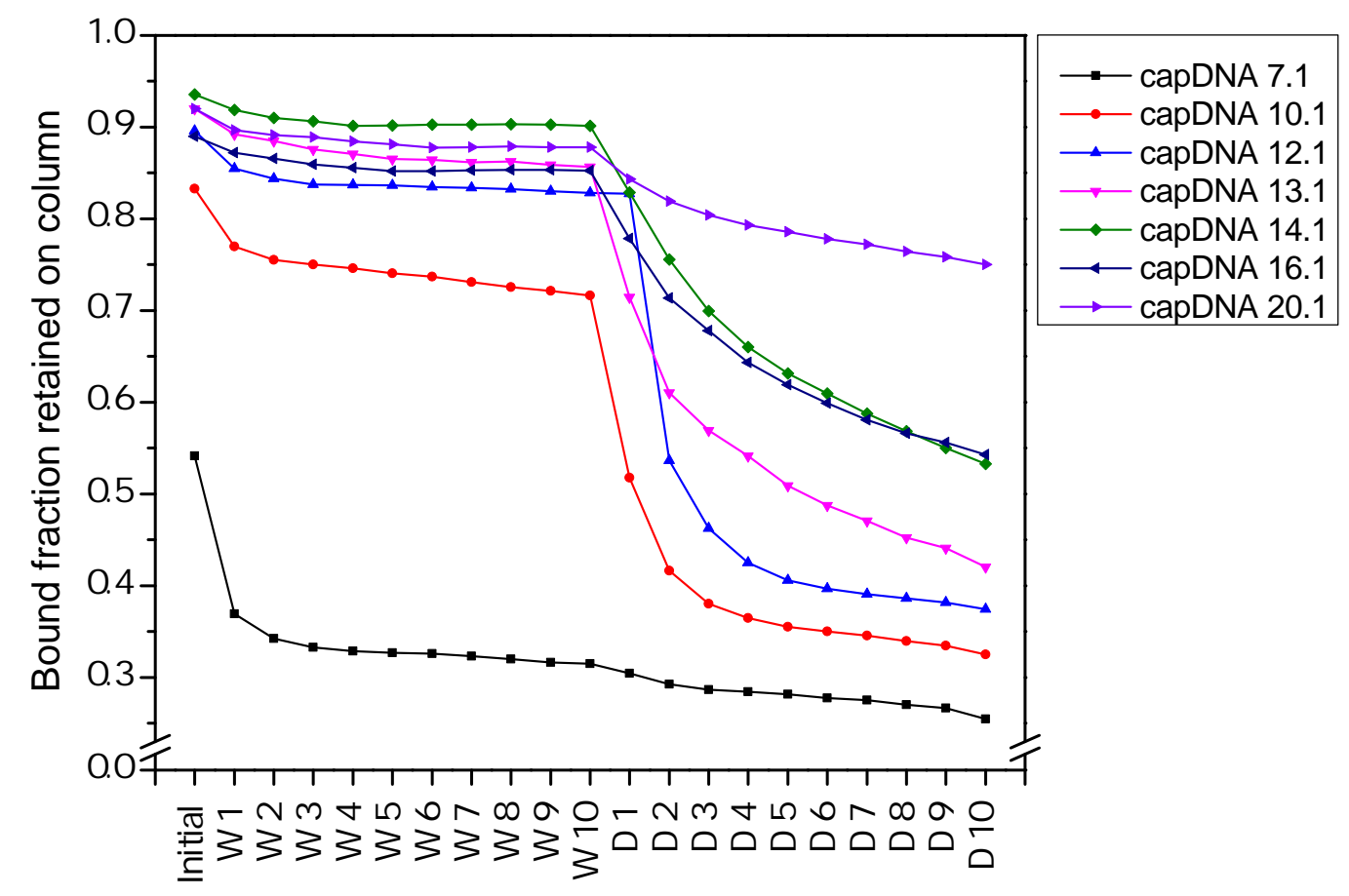

Figure 3.6: Fraction of bound ssDNA to seven different capture oligonucleotides remaining on the column after several washing steps. 'Initial' indicates the retained amount of DNA directly after incubation, W1 to W10 the amount of DNA after DSB washes of one column volume each and D1 to D10 after the induced release of DNA by one column volume washes with denaturing buffer. 


\subsubsection{Optimisation of Amplification Procedure}

\subsubsection{PCR Parameters}

Since in the first rounds of selection the use of Q5 hot start high-fidelity polymerase was intended, a first step was to optimise the PCR parameters, concerning specifically annealing temperature and SybrGreen I concentration for that specific polymerase. As a measure the maximum relative fluorescence unit (RFU) value was divided by the threshold cycle (ct) to indicate a fast amplification (low ct-value) and a high amount of product at the end of amplification (high maximum RFU value). Figure 3.7 A shows the result of the amplification with the Q5 polymerase. With respect to SybrGreen I a concentration of $0.3 \mathrm{x}$ and $0.4 \mathrm{x}$ appears optimal. As the SybrGreen I containing reactions should also be included for further SELEX rounds, the lower concentration of $0.3 \mathrm{x}$ was used in some reactions to monitor the amplification procedure on-line. Furthermore, higher temperatures are prone to lead to better results, particularly $58^{\circ} \mathrm{C}$ and above. As the PCR instrument allowed the use of different annealing temperatures, a gradient of 57 to $61{ }^{\circ} \mathrm{C}$ was chosen, with respect to the unknown diversity of the pool. $\mathrm{MgCl}_{2}$ was already included in the delivered buffer, thus the concentration was used as supplied. Similar results were obtained with the FastStart Taq polymerase from Roche (cf. Figure $3.7 \mathrm{~B}$ ). Here, a $\mathrm{MgCl}_{2}$ concentration of $2.5 \mathrm{mM}$ was used as it gave the best results. The annealing temperature program from the former polymerase was kept.

\subsubsection{Double Strand Separation}

After PCR and cleanup, strand separation was done by digesting the reverse strand with Lambda Exonuclease. This method is advantageous compared to alkaline denaturation and magnetic bead separation, leading to higher yield, efficiency and purity [184]. Lambda Exonuclease is an exodeoxyribonuclease with 5 ' to 3' activity, catalysing the removal of 5' mononucleotides from duplex DNA. 5'-phosphorylated double strands are thereby highly preferred.

Fig. 3.8 shows large bulk amplification and digestion of the library with Lambda Exonuclease at different incubation times. After PCR and cleanup (Qia) a clear band is visible at the target size of $78 \mathrm{bp}$. A second band at about half 
A

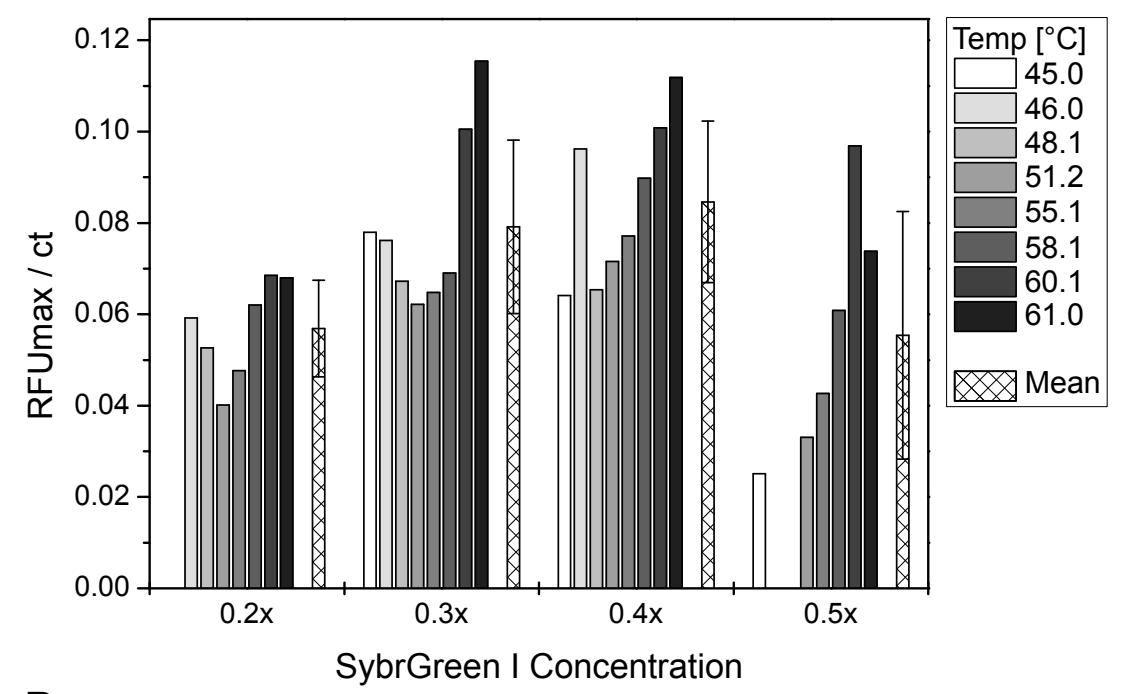

B

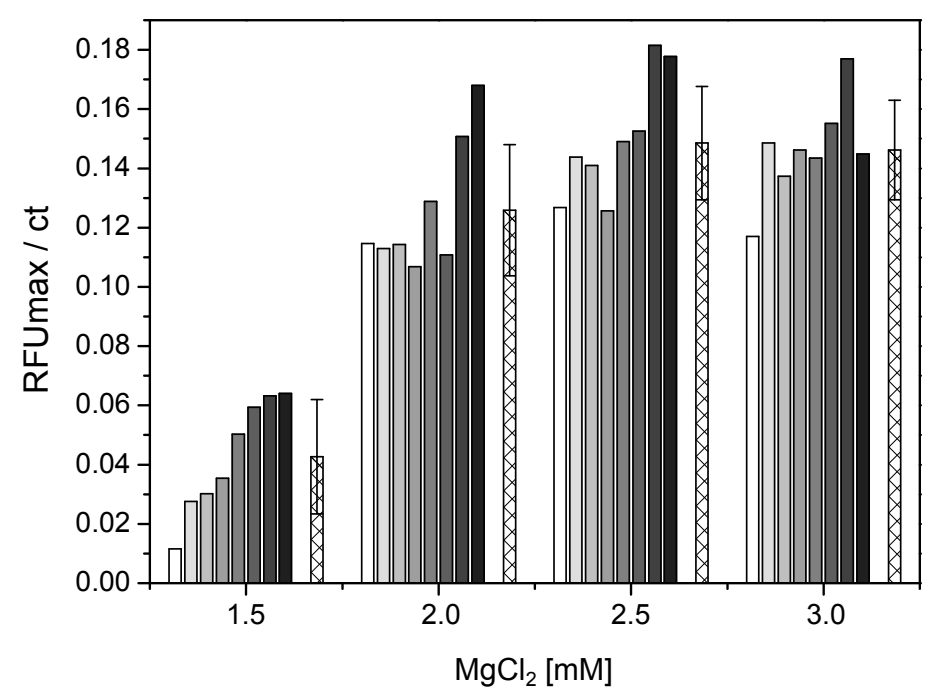

Figure 3.7: Optimising the PCR parameters. A: Estimating the annealing temperature and the SybrGreen I concentration for Q5 High-Fidelity polymerase. B: Annealing temperature $\mathrm{x} \mathrm{MgCl}_{2}$ concentration of FastStart Taq polymerase. The error bars of the mean value are indicating standard deviation.

of the size of the upper band appears after 20 min of Lambda Exonuclease treatment. Due to a digestion of the phosphorylated reverse strand, the single forward strand remains. With increasing incubation time the template double-strand disappears while the single-strand increases. After $80 \mathrm{~min}$ the template DNA is 
almost totally digested. Thus, an incubation time of 90 min was chosen for the SELEX experiment, to ensure complete digestion.

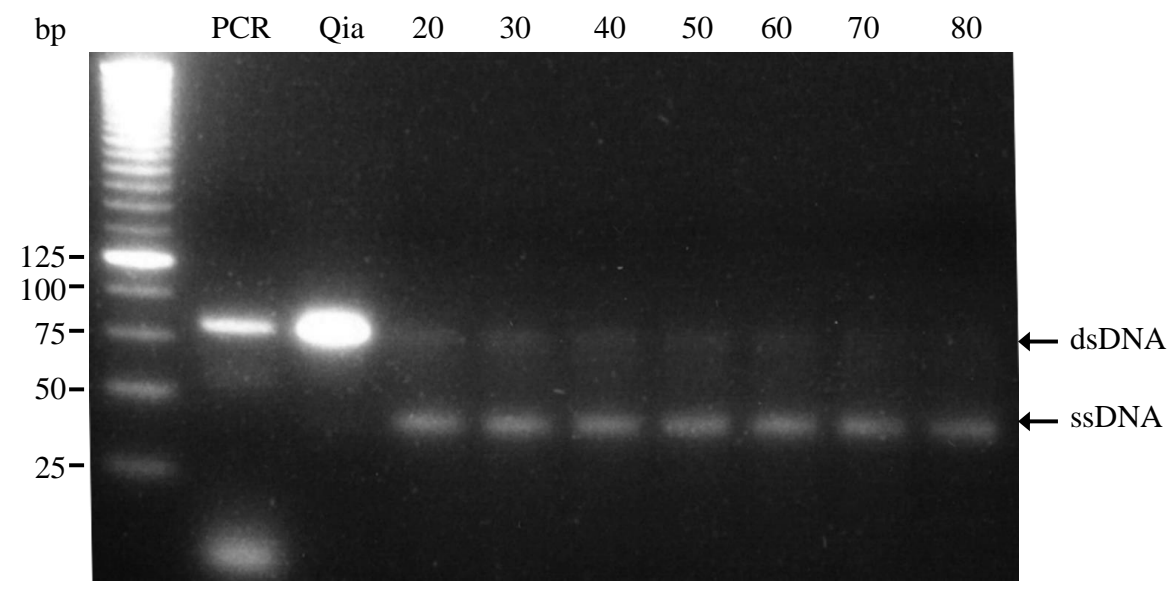

Figure 3.8: Agarose gel electrophoresis of strand separation test. PCR: PCR amplified library; Qia: Cleanup of amplified library via Qiagen PCR purification kit (3x concentrated); 20-80: Minutes of Lambda Exonuclease digestion of purified amplicon.

\subsubsection{SELEX Experiment}

The selection was monitored via the attached fluorophore. Figure 3.9 shows the eluted ssDNA over the rounds of selection.

From the first round of selection, the eluted ssDNA started to accumulate. To avoid the accumulation of unspecific aptamers, the first negative selection step was introduced in round 4. The amount of DON-specific DNA decreased thereupon, but again increased through rounds 5 and 8 up to $8 \%$. The second negative selection step, starting in round 9 , caused a breakdown again, indicating the presence of some unspecific DNA in the pool. In the ongoing selection the DNA accumulated again until round 12. This round also marked the maximum of selection. Indeed, the absolute amount of DNA further rose in round 15 and 16, but the large increase is due to the increased ratio of elution volume to column volume from 23 to 35 in round 15, as confirmed by the elution volume-corrected value. A reason for the decline in rounds 13 and 14 might be overselection. Thus, 


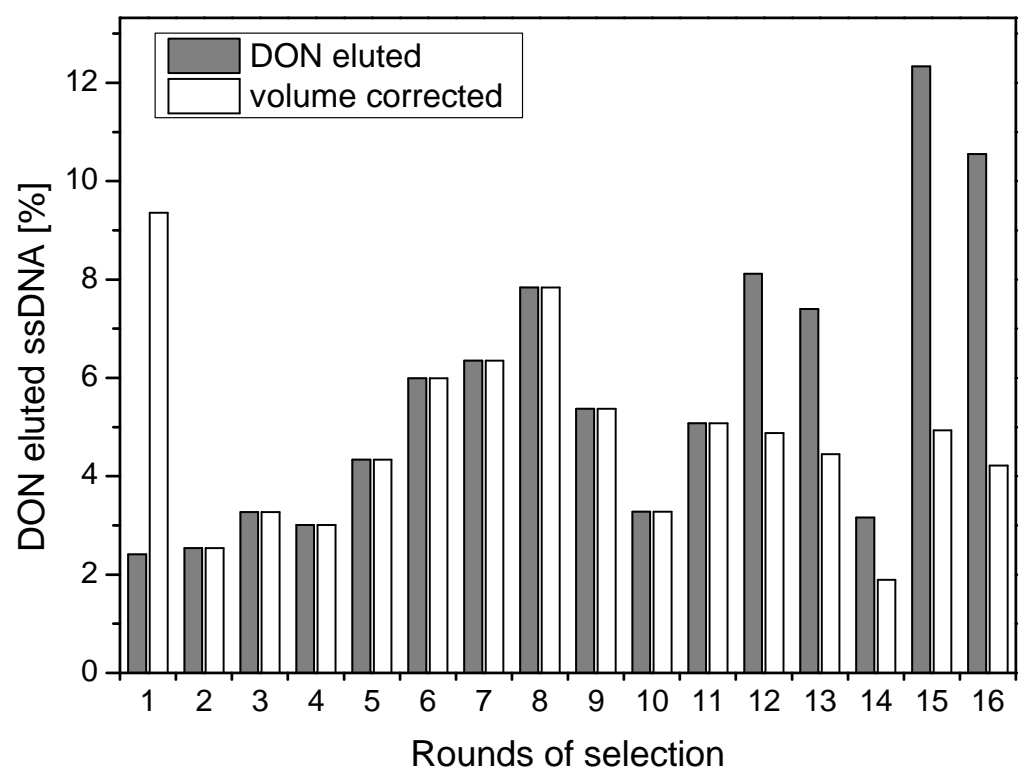

Figure 3.9: Eluted ssDNA during different rounds of selection. Black bars show the percentage of ssDNA specifically eluted by $2 \mathrm{~mm}$ DON. White bars show the eluted ssDNA elution volume corrected.

the pool of round 12 was chosen for a detailed analysis of contained aptamers. Thereto, the pool was cloned in E. Coli. 117 colonies were picked and pDNAs were extracted. Therefrom 106 clones (90\%) contained the insert (cf. Figure 3.10). This resulted eventually in 76 sequences.

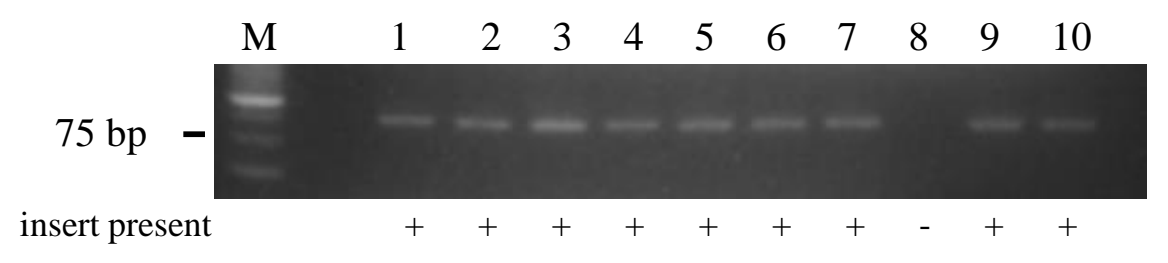

Figure 3.10: Exemplary agarose gel electrophoresis of EcoR I digested pDNAs from 10 out of 117 individual clones. M: Size marker, 25 bp each.

While $91 \%$ of the analysed structures had the correct length of the initial library, there were four aptamers with a length of only 77 nucleotides. Also longer chains were observed. Two clones had a length of 79 and one (clone 50) 
had a chain length of 81 nucleotides.

As expected, in most of the clones, the first 12 bases (starting from 5' end) were involved in forming the secondary structure as this was intended by the selection mechanism. In numbers, the probability that at least six nucleotides of the first 12 were existent as double strand is on average $83 \%$ and within 49 out of 76 clones it is higher than $90 \%$. In contrast, the probability for at minimum six double-stranded nucleotides in the 3 ' constant region is on average $10 \%$ with only 4 out of 76 clones higher than $90 \%$. This indicates that the 3' region is only rarely used for structure forming.

\subsubsection{Characterisation of Selected Aptamers}

39 of the sequences from the selection were chosen and tested separately on DON binding in a binding assay. 14 sequences reacted positive upon DON incubation and are shown in Figure 3.11.

The $\triangle \mathrm{RED}$ ranged from 0.02 to 0.2 , meaning that the relative DON eluted DNA from the best binder (c41) was on average $20 \%$ higher than in the associated control. However, the variation was in parts very large. Coefficients of variation $(\mathrm{CV})$ were between $0.11(\mathrm{c} 8)$ and 6.4 (c65). Consequently, the aptamer c8 showed with 0.10 a $\triangle R E D$ value which is significantly ( $\mathrm{p}<0.01$, one-sample t-test) different from zero. If several aptamers were combined (Set 1 to 3 ) they additionally showed extremely significantly ( $\mathrm{p}<0.001$, one-sample t-test) higher DNA elutions in the presence of DON than without. For that purpose it was irrelevant which aptamers were aggregated. Set 1 only contained the best binders (c8, c15, c77 and c41) with respect to variation. Set 2 was broader and contained additionally the high $\triangle$ RED-valued aptamer c32 as well as c42, c29 and c18. Whereas set 3 comprised all of the 14 best binders. Each of the sets were thereby significantly different from zero, indicating that they are susceptible for the application of DON, although the variation is high.

It has to be mentioned that the individual $\mathrm{RED}_{C}$ values of the aptamers differed significantly ( $\mathrm{p}<0.05$, two-sample t-test) from the library. While the control of the library eluted on average $6 \%$ of DNA during incubation, the unmodified aptamers eluted by mean about $19 \%$. This indicates, that the aptamers are in 


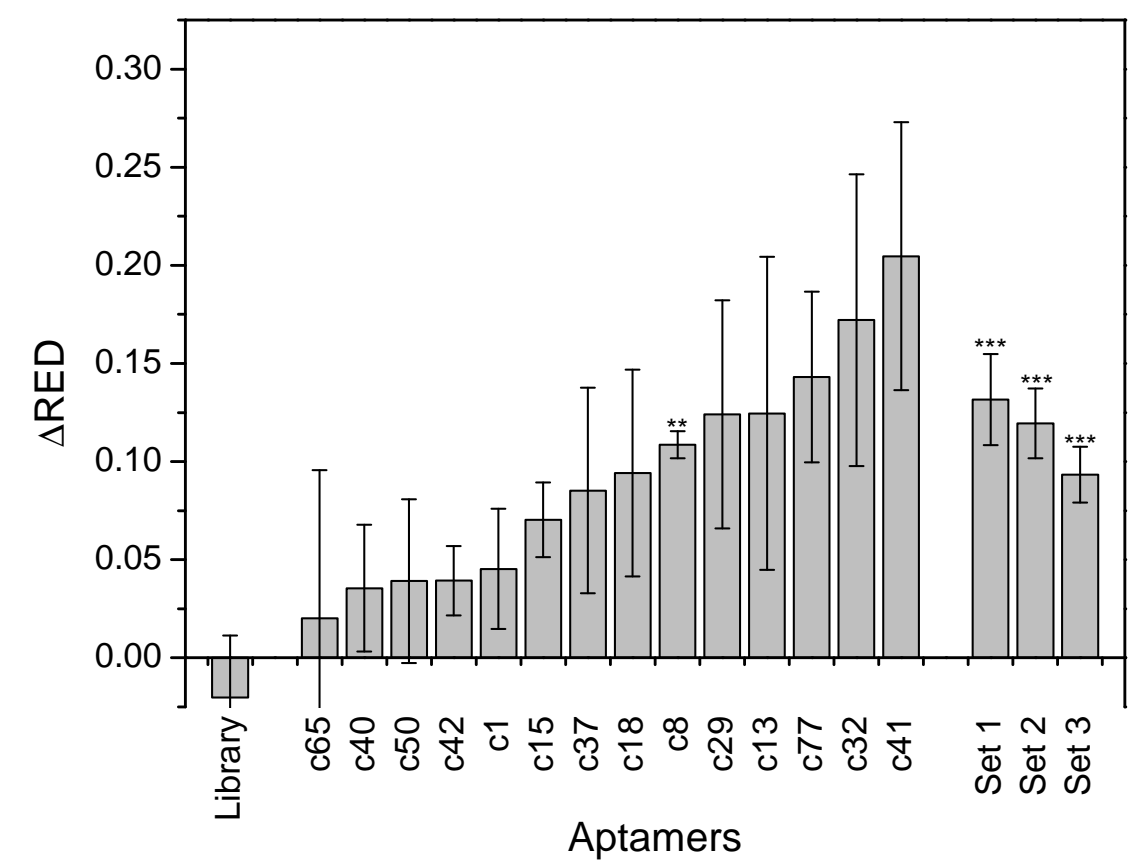

Figure 3.11: Binding assays with the TOP-14 aptamers $(n=3)$ as well as the pure library $(\mathrm{n}=3)$ and aggregated sets. Set 1 comprises aptamers with a $\mathrm{CV}<0.6$ $(\mathrm{n}=12)$; set 2 aptamers have $\mathrm{CV}<1 \quad(\mathrm{n}=24)$ and set 3 is containing all TOP-14 aptamers $(\mathrm{n}=42)$. A detailed description of the sets is given in the text. $\triangle \mathrm{RED}$ is the difference of $2 \mathrm{~mm}$ DON-eluted-DNA and a control. Error bars show standard error. ${ }^{* *} \mathrm{p}<0.01,{ }^{* * *} \mathrm{p}<0.001$, one sample t-test against zero. 
general less capable of binding to the affinity column, since they form a threedimensional stable structure in contrast to the species from the library.

The Figures 3.12 and 3.13 are representing the secondary structures estimated for the aptamers with a CV-value lower than 0.6 and 1 , respectively. The structures with the minimum free Gibbs energy $\Delta \mathrm{G}$ out of 100 calculated possibilities are shown. The individual minimum $\Delta \mathrm{G}$ values of the aptamers can be found in Table 3.5. The given probabilities indicate on a nucleotide basis how well the given structures coincide with the non-shown ones and thus if they are fluctuating within the calculations or not. In most of the cases the structures have two large domains, with one to three interior loops and a hairpin structure at the end. The aptamer c8 stands out. The whole structure is involved in forming one large domain and the given arrangement is only a rough estimate of the real secondary structure due to the low pairing probabilities, although it has good DON binding properties. It is remarkable, that all motifs are based either on the 5' or 3' constant region. As the selection mechanism was focused on the 5' region, this domain is in many aptamers more distinctive. The 3' domains are rather short, unstructured and poorly determined with the exception of c77. Thus, it was analysed if the removal of these structures shows any differences in binding properties. 
A
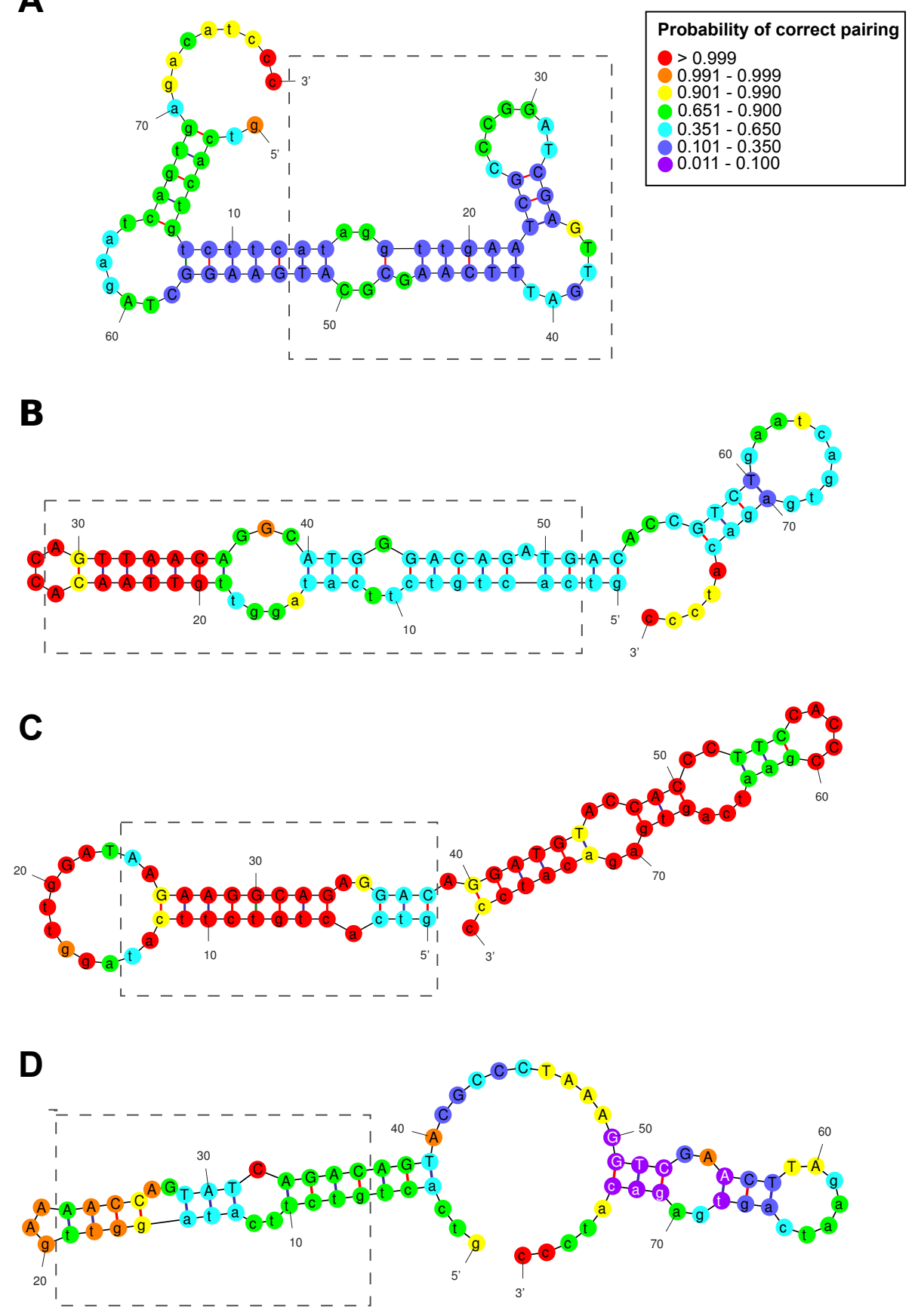

Figure 3.12: Predicted secondary structures of the TOP-4 aptamers c8(A), c15(B), c77(C) and c41(D) in terms of variation, covering the CVs of $0.11,0.47$, 0.53 and 0.58 , respectively. The structure with the minimum free Gibbs energy $\Delta \mathrm{G}$ is shown here. Circle colours indicate the probability of correct (double/single) pairing of each nucleotide. The 5' fluorophore-label is not shown. Small letters indicate the constant regions. Dashed-rectangles mark the motifs, defined in Table 3.5. 
A

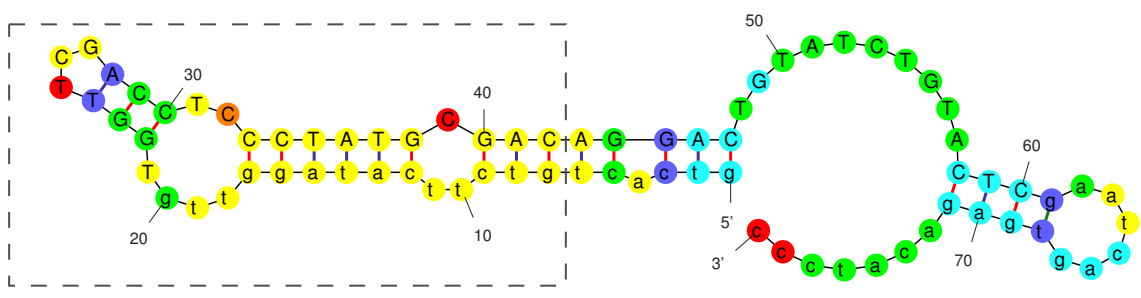

B

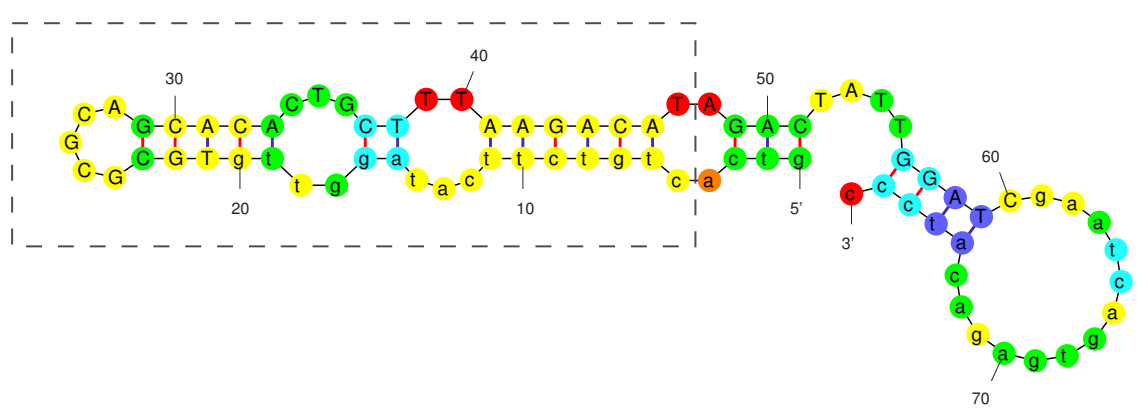

C
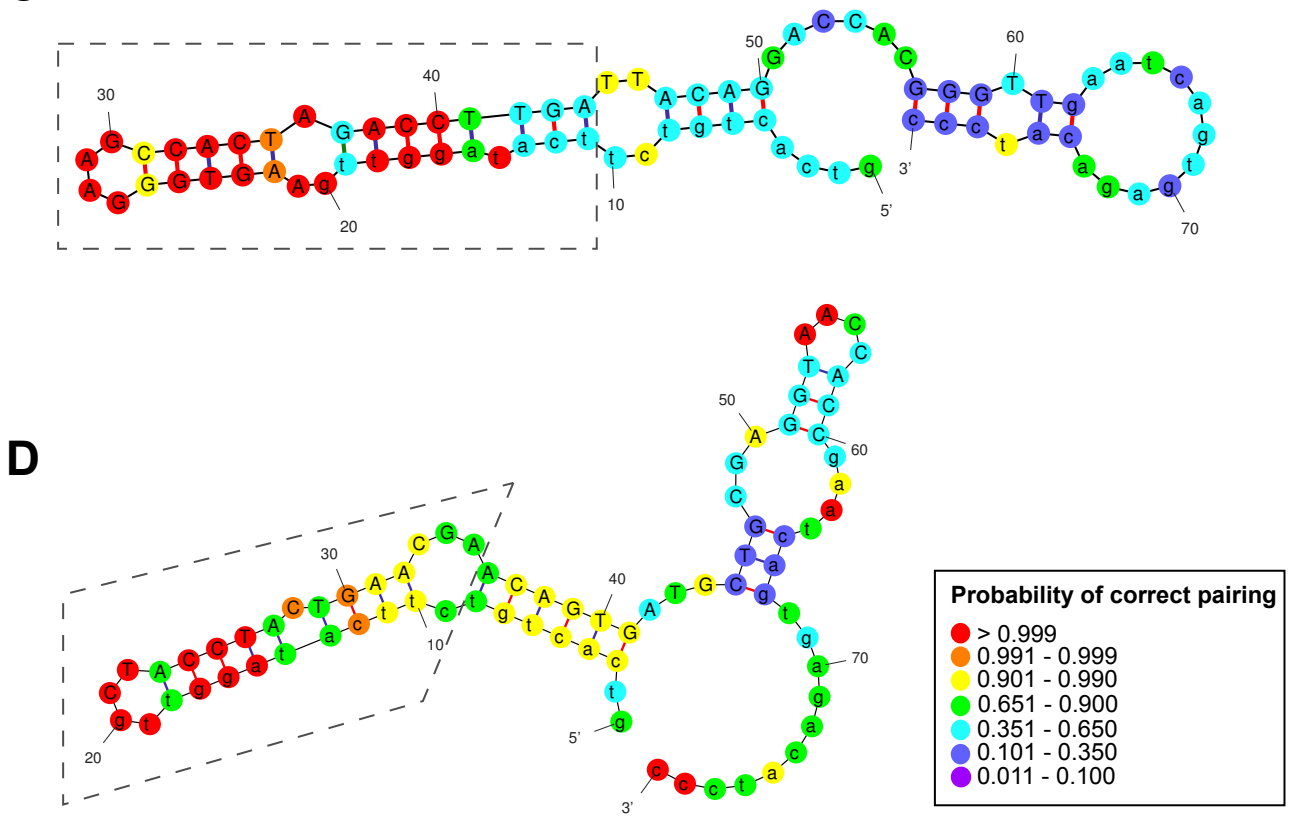

Figure 3.13: Predicted secondary structure of the aptamers c32(A), c42(B), c29(C), c18(D). The CVs are $0.75,0.78,0.81,0.97$, respectively. The structure with the minimum free Gibbs energy $\Delta \mathrm{G}$ is shown here. Circle colours indicate the probability of correct (double/single) pairing of each nucleotide. The 5' fluorophorelabel is not shown. Small letters indicate the constant regions. Dashed-rectangles mark the motifs, defined in Table 3.5. 
In a first step the nucleotides from the random region, which were not involved in forming the 5'-domain were removed (c32m1, c37m1, c41m1, c77m1). Besides the modification of $\mathrm{c} 32 \mathrm{~m} 1$ there was an additional thymine incorporated at the 45th position to match with the non-paired adenine from the 4 th position to check whether the aptamer folds into a more stable structure $(\mathrm{c} 32 \mathrm{~m} 2)$. Additional to c $77 \mathrm{~m} 1$ the guanine at the 30 th position was exchanged with an adenine to remove the wobble base-pair $(\mathrm{c} 77 \mathrm{~m} 2)$. As all these modifications showed a huge decline in the binding assay (cf. Figure 3.14), the two aptamers c77 and c41 were drastically cut at the positions $38(\mathrm{c} 77 \mathrm{~m} 3)$ and $42(\mathrm{c} 41 \mathrm{~m} 2)$ to check whether the complete removal of the tail results in any change. Thereupon, a reaction on DON for both aptamers could be determined. However, the $\triangle \mathrm{RED}$ mean value was much lower than in the non-modified versions (cf. Figure 3.14).

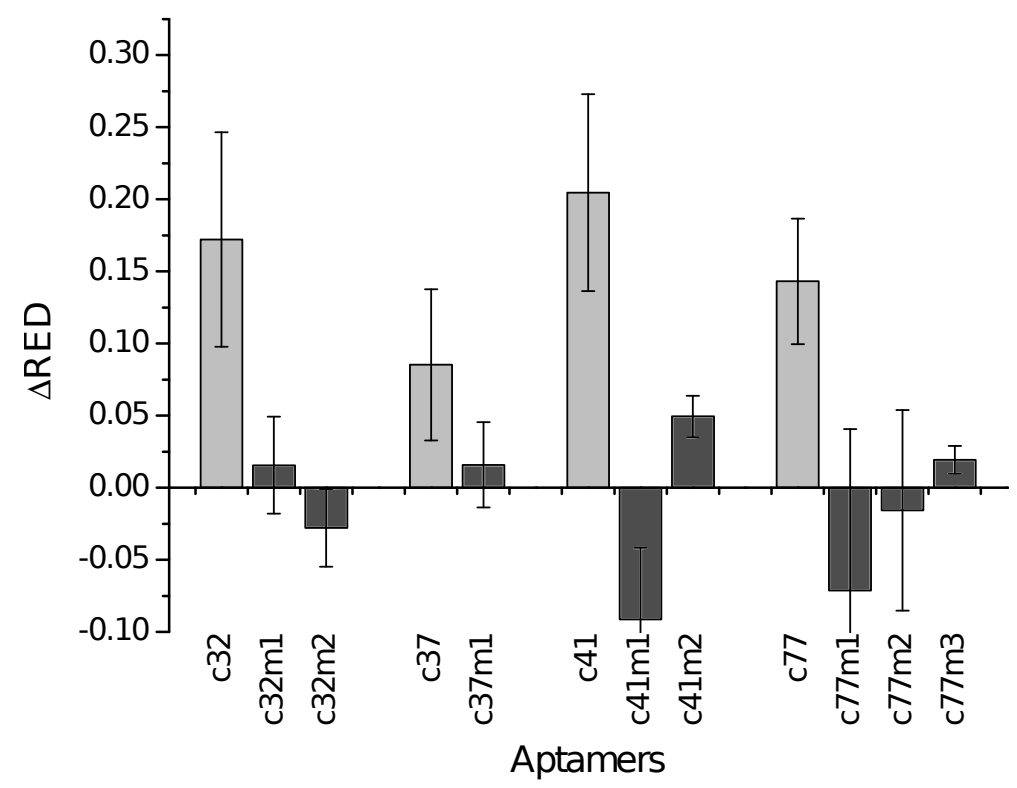

Figure 3.14: Binding assays of the modified aptamers as well as their corresponding initial type. Error bars show standard error $(\mathrm{n}=3)$.

Table 3.5 shows the primary sequences of the 14 highest, DON-dependent eluted aptamers. They can be grouped by common motifs of 7 to 28 nucleotides. Group one as well as group two are comprising the most well eluting aptamers. Again c8 does not fit in these groups. It is rather similar to c42 in terms of a common motif, but not in DON-dependent elution. The existence of common 
motifs is rather unsurprising, since the motif-region is often complementary to the 5' constant region which acts as a template. However, it is noticeable that single-bulge loops occur most often in the motifs of group 1, 2 and 4, specifically in the aptamers of c41, c32, c77, c29 and c15. These small caves can probably act as binding site for DON as they offer the ideal size of about one nucleotide. The GC-content is in the range of 44 to $55 \%$ with an average of $49 \%$. This is exactly the mean GC-content of the library, assuming that the variable region was completely randomised. Hence, there were no sequences preferred in the SELEX due to special nucleotides (e.g. guanine-rich sequences). 


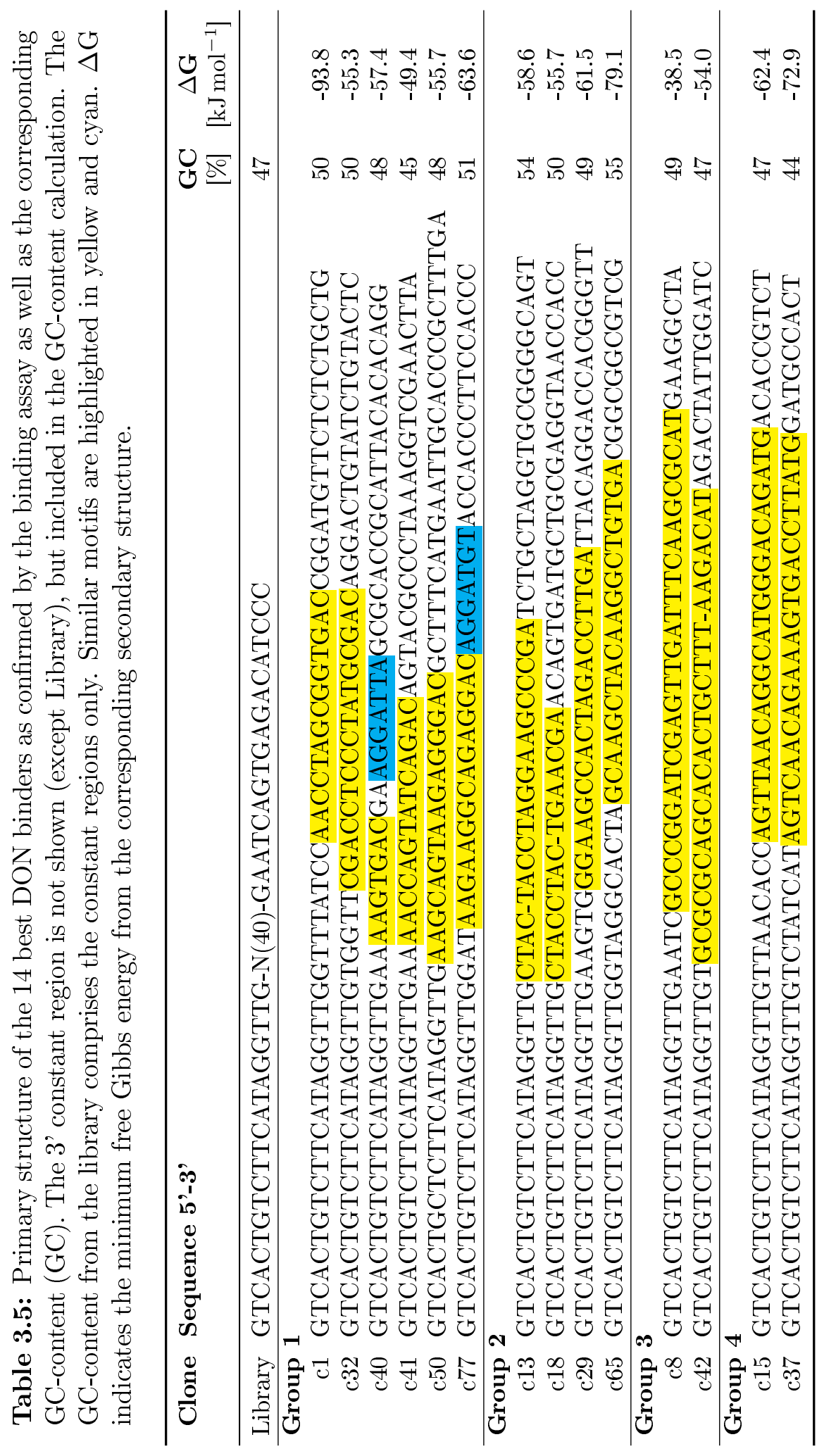




\section{Chapter 4}

\section{Discussion}

\subsection{Volatile Organic Compounds}

In a first experiment the volatiles released by different Fusarium fungi were analysed. Some substances such as ethanol or hexadecane appeared in all samples, several other compounds, previously described as infection-specific, such as 2-methyl-1-propanol, 3-methyl-butanol, 1-octen-3-ol and 3-octanone [40, 46, 186] were differentially detected in some samples but never in all. Specifically, 1-octen-3-ol is known to be a fungal biomarker [38, 40, 41, 46, 186, 187]. Accordingly, it was emitted from the Fusarium infected samples, but not in the control. In contrast, Becker [43] found that 1-octen-3-ol is released from uninfected wheat samples and reported reduced contents with respect to fungal infection. In agreement with this study 3-octanone was only detected in the F. graminearum probe. However, other studies brought evidence that this substance is discharged from F. sporotrichioides [38] and other fungi [188-190]. Moreover, hexanal is widely found in relation to agricultural products including milk [191], chicken [192], melons [193] and fungi [194]. Consistently, it was also found in all samples from the present study and is thus not directly related to Fusarium infestation. The precursors for 1-octen-3-ol, 3-octanone and hexanal are the linoleic and linolenic acids [194], present in many living plants and organisms.

In the current study, different substances such as benzeneacetaldehyde, p-xylene, 2,2-dimethyl-1-propanol and butyrolactone were identified as specific to different 


\section{DISCUSSION}

Fusaria species. Some of these substances were also found in relation to airborne fungi [50, 195, 196], but not to Fusarium spp.. The sophisticated occurrence of different MVOCs with respect to fungal contamination is probably a consequence of nutritional effects, as MVOCs are formed in the primary and secondary metabolism of microorganisms [194]. Moreover, the methodology of the study plays also an important role, as some refer to in vitro $[38,43,46]$ and others to in vivo $[43,45]$ experiments. This can heavily influence the odour composition since living plants release accessory volatiles upon fungal attack [43]. The choice of the method depends on the desired application and thus on the question weather the fungi should be identified in storage or on the field.

Among the various substances suggested to act as volatile biomarkers for Fusarium infection, none of them turned out to be specific for certain Fusaria species or strains [43]. The identification of risky Fusarium species is absolutely necessary for discriminating potentially hazardous grain from harmless ones and to avoid the disposal of false positive samples. In addition, FHB is usually related to the occurrence of different Fusarium species, which leads to a more complex odour composition and makes species identification challenging. Thus, it seems to be the pattern of chemicals that is characteristic for each species. Such patterns can be detected by using an electronic nose [197], i.e., an array of solid-state sensors that are non-selectively sensitive to the relevant chemicals and whose responses reflect the chemical information contained in the sample. This detection scheme is in many respects similar to natural olfaction where hundreds of different receptors allow to distinguish among tens of thousands of different odours [198].

\subsection{Electronic Nose Recognition}

An electronic nose was used to discriminate between four different Fusarium species on wheat grain, including the widespread mycotoxin producers F. graminearum and F. culmorum. The Enose consisted of an array of metalloporphyrincoated QMB sensors, each being sensitive to a range of molecules. In a number of studies, porphyrins have been shown to be suitable for chemical sensing [179, 199, 200] and as coating material for QMB-based electronic noses [119, 201, 202]. Enoses are fundamentally dependent on the sensing properties. Metallopor- 
phyrins in particular are convenient as coating material, since they offer a wide variety of opportunities for controlling the sensor selectivity [119]. Along the line, such Enoses have been approved in quality control of agricultural products [125, 126, 129, 201].

The Enose analyses performed at $30{ }^{\circ} \mathrm{C}$ turned out to be more reproducible than those performed at $70^{\circ} \mathrm{C}$ (data not shown). This somewhat unexpected behaviour may have the following explanations. While transferring the gas from the sampling vial to the sensor array, the temperature was not kept constant. A temperature drop might have induced the condensation of some volatile compounds, resulting in a change of the composition of the sample prior to entering the sensor cell. In addition, the adsorption of volatiles onto a porphyrin layer is a temperature-dependent process, the efficiency of which decreases with increasing temperatures. Several studies report sampling temperatures in a range of 30 to $40{ }^{\circ} \mathrm{C}$ [129, 130, 203], confirming lower temperatures as more suitable for Enose analysis.

In the present study, the electronic nose was able to discriminate between infested and non-infested grain with an accuracy of $83 \%$. Moreover, it could distinguish between two levels of infections and between different species with classification rates of $91 \%$ and $94 \%$, respectively. However, $18 \%$ of highly infected samples were classified as low infected. This appears to be the result of the simple, linear classifier. A more complex classifier such as a neural network would presumably improve the classification rate. However, there is a negative correlation between the classifier performance and the generalisability of the results. If the complexity of the classifier is kept low, the results are usually more general, whereas results obtained with more complex classifiers tend to be less prone to generalisation. Falasconi et al. [130] compared the results of an Enose measurement of Fusarium verticillioides-infested corn with respect to the classification method. A simple linear discriminant analysis (LDA), which is similar to PLS-DA, led to a rate of $94 \%$ correct classification of contaminated vs. noncontaminated corn. In contrast, a $k$-Nearest-Neighbour analysis revealed a correct classification of $99 \%$. For this study, a simple and robust classifier was preferred. A more complex analysis, whose results can at the same time be generalised, is desirable and would certainly be preferred in many cases. 


\section{DISCUSSION}

The false negatives observed in the classification of infested and non-infested grain originated all from $F$. redolens at high infection level. The variance of this group might be explained by the fact that the high concentrations of those samples were associated with changes in the relative components of the patterns. These probes also led to false classifications during species identification. Furthermore, the data of the infected group showed a rather large variance, most presumably due to the heterogeneity of the dataset consisting of probes from different Fusarium species and at different levels of infection. The probes falsely classified as infected are brought about by a high variance in the control group. The variance is caused by several factors. As the model was chosen to perform a binary classification, it was unable to differentiate between species or levels. Moreover, the control group contained samples exposed to different incubation times and the incubation of non-inoculated grain may have changed the composition of odour compounds. In practical terms, false negative classifications would seriously compromise the Enose approach, while false positive classifications were considered acceptable, though unsatisfying. The results are in accordance with findings from Paolesse et al. [129] and Balasubramanian et al. [204] who showed classification rates of $85 \%$ and $87 \%$ on the analysis of Fusarium on wheat or barley. Thereto, PLS-DA and LDA discrimination was used in these particular studies.

The present study aimed at a qualitative recognition of Fusarium-infested grain with the future intent to reject infected grain from being processed as feed or food. This approach was done with respect to the possible mycotoxin contamination related to such cereals. Others tried to quantify mycotoxins in grain or flour directly, using an electronic nose [131, 133]. However, such methods are generally semi-quantitative and relay on the uncertain correlation of Fusarium infection and mycotoxin production.

\subsection{Aptamer Development}

Aptamers are usually selected in a SELEX experiment. Hence, a library of oligonucleotides is incubated with the target molecule. In the selection step, the oligonucleotides bound to the target are separated from those which are not 
bound. This step is the most crucial aspect in the selection process as it determines the binding features of the prospective aptamers. Here, methods which relay on target immobilisation (e.g. magnetic bead separation) [142, 163, 170, 205] or size separation (e.g. filtration, centrifugation) [206] are commonly used. Although these assays are successful in many cases, they have failed so far to select aptamers against deoxynivalenol due to several possible reasons. Firstly, DON has to be modified chemically. As it is a very small molecule (296 Da), modifying it without altering the whole structure is challenging. Secondly, by immobilising the target, binding sites are covered and blocked for potential aptamer binding. Thirdly, the mass of small molecules is almost negligible in comparison to the individual DNA molecules from the library, leading practically to no change of mass during complex formation. Thus, separations with respect to the change of mass (e.g. with capillary-electrophoresis-SELEX) are not practicable.

In the present study, an in vitro selection of structure-switching aptamers, upon DON binding, was applied. A single-stranded DNA library covering 40 complete random positions and theoretically $6.6 \times 10^{14}$ different molecules, was utilised. The length of the random region was chosen with respect to the target molecule and based on literature. DNA aptamers possessing core regions of 20 to 60 bases were often reported (cf. Table 1.5). A longer core region theoretically results in a higher complexity of the library. However, practically, only a small portion of the maximum complexity can be tested in the selection. Furthermore, the smaller the molecules of the library are, the larger is the proportion of target-mass to the library, resulting in a higher mass change upon target binding. Complete random-sequence libraries cover a much wider sequence space and are suitable for the isolation of novel binding species [207]. A DNA library was chosen in the current study as it is much less prone to degradation and easier to amplify. It has been shown, that DNA aptamers can bind ligands in the same extent as RNA aptamers, although the structures are completely different [148].

The separation process was based on a conformational change of the oligonucleotides of the library. Therefore, the library was reversibly bound to an affinity column by partially hybridising to a capture oligonucleotide. This approach was successfully used for generating aptamers binding on different nucleotides and zinc-ions [143, 145, 152, 208]. The procedure is advantageous for the aptamer- 


\section{DISCUSSION}

selection against DON, since DON-molecules do not need to be modified chemically. The strength of the interaction of the library to the column is accurately defined by the length of the hybridised, complementary region and defines the extent of the conformational change necessary for DNA release. If the affinity is too high, the structure-switching upon DON binding will not take place and the relevant species will not be eluted. If the affinity is too low, unspecific species are also eluted and amplified, lowering the efficiency of the SELEX-process. Stevens et al. [209] estimated a dissociation constant of less than $1 \times 10^{-11} \mathrm{M}$ for a DNA hybrid of 22 nucleotides. Smaller hybrids are thus less bound to the column. For 14 and 15 complementary nucleotides, dissociation constants of $2 \times 10^{-9} \mathrm{M}$ and $7 \times 10^{-10} \mathrm{M}$ have been evaluated [210]. However, the primary sequence as well as experimental conditions (temperature, $\mathrm{pH}$, buffer composition) have an influence on the binding-affinity. Therefore, an experiment was conducted to estimate the optimal length of a capture-oligonucleotide in a range of 7 to 20 nucleotides. According to literature [143] a chain length of 12 bases showed an optimum between capturing the library on the column and releasing it upon disruption of the complementary interaction.

During SELEX, the DNA species binding to DON and thus undergoing a structure-switching were released and eluted from the column. The selection buffer was chosen with respect to the negative backbone of the DNA and the relative polar structure of DON. It has been shown, that metal ions can stabilise the tertiary structure of RNA [211]. Negative charge repulsions may inhibit

the formation of a complex structure. Positive ions such as $\mathrm{Na}^{+}$or $\mathrm{Mg}^{2+}$ are equalising these charges and were used to stabilise the DNA. Furthermore, it has been shown, that high affinity aptamers are less magnesium dependent [212]. Hence, a relative low magnesium content was applied.

After the selection, the eluted DNA must be amplified for a new round of SELEX. Here, it should be considered that the template DNA is completely heterogeneous. Such DNA is extensively susceptible for PCR-overamplification. In contrast to end-point PCR, the product formation stops when PCR primers are still in excess. Five to ten rounds of amplification above the optimal cycle number can lead to a complete loss of the specific dsDNA products due to by-product formation [213]. However, a low cycle number results in a small amount of product 
and a low copy number. Therefore, generally large-scale PCR is carried out with multiple reactions [207]. In the present study, PCR was optimised concerning $\mathrm{MgCl}_{2}$ and SybrGreen I concentrations. When an overamplification took place, a second band was visible in the gel-electrophoresis (cf. Figure 3.8, lane "PCR"). Accordingly, online monitoring of the PCR process was carried out for 6 out of 30 reactions during the SELEX experiment. The amplification was stopped as soon as 60 to $90 \%$ of the maximum amount was reached. Consequently, there was no second band visible in the amplifications of the SELEX.

Once the selected DNA has been amplified, it is necessary to perform a strandseparation as PCR products are commonly double-stranded, although asymmetric PCR can provide ssDNA [214]. However, this type of PCR is less efficient as amplification occurs almost linearly. Therefore, strand-separation was done by Lambda Exonuclease-digestion of an phosphorylated reverse strand [184, 215]. Lambda Exonuclease is an enzyme induced by bacteriophage lambda. It has strict 5 ' to 3 ' activity and digests as an exodeoxyribonuclease double-stranded DNA in a 350-fold higher extend as single-stranded DNA, releasing 5'-mononucleotides [216]. It has a strong preference for 5' termini carrying a phosphoryl residue [216]. This procedure is beneficial compared to alkaline denaturation, leading to higher yield and purity without having interfering molecules such as streptavidin or biotin [184].

During rounds of selection against DON, an increase in eluted ssDNA could be observed. This rise yielded a local maximum in round 8 and decreased thereupon. The setback is reasonable since a second negative selection step was introduced in round 9, indicating that there were still many unspecific binders present in the pool. After subsequent rounds of selection and amplification a second local maximum occurred in round 12 with a DON-induced elution of $8 \%$ of ssDNA. As the column volume was reduced in round 12 , the absolute elution, corrected for the volume, was smaller than this from round 8. However, as the selection was going four rounds further and many unspecific binders were additionally removed, this pool was chosen for a detailed aptamer analysis. The further drop in the subsequent rounds can be explained by overselection, as it has been observed in many cases $[142,163,206]$. The large increase in round 15 originated from the reduced column volume. However, starting from round 12, no further rise 


\section{DISCUSSION}

was observed with respect to the column volume-corrected values. Rajendran and Ellington observed maximum elutions of $34 \%$ and $12 \%$ after nine rounds of selection by applying the same method [143, 145], with the first study being a selection against $\mathrm{Zn}^{2+}$ ions. However, it is well known, that ions interact with DNA, supporting the formation of complex structures. Others selected aptamers successfully with a maximum elution of $2.2 \%$ [163] or $7.5 \%$ [142] during SELEX.

\subsection{DON Aptamers}

The structures which were got from the SELEX had mostly the desired length of 78 bases. However, some were shorter and even longer. There are two possible explanations for that observation. The library could have been contained mutations in chain length due to incorrect synthesis. Although it has been HPLC purified and tested in a gel-electrophoresis, a heterogeneous, random library will always give a broader band [207] were small differences in chain length will not be detected. Moreover, if there were only few molecules affected, they would be below the detection limit. This reason is somehow unlikely as exactly these sequences needed to be selected and amplified, though possible. In addition, as the PCR is generally susceptible for mutations it is most likely that the variations in chain length occurred during amplification of the polymerase. To reduce the mutation rate a high-fidelity polymerase with a 100 -fold lower error rate was chosen in the first rounds of selection. Nonetheless, smaller products can occur due to a formation of highly stable stem-loops in GC-rich sectors providing jumping of the polymerase [217]. As the library was already randomised, additional variation was not intended to bring into the SELEX. However, the mutations also underlay the evolutionary selection and should not worsen the result.

To analyse the individual aptamers on their DON-binding capability an assay was performed, similar to the selection experiment. Therefore, the particular sequence was bound to an affinity column and eluted through DON. The eluted amount was then compared to a control were the elution took place with buffer $\left(\mathrm{RED}_{C}\right)$. It has to be noted that the $\mathrm{RED}_{C}$ value differed between the library and the aptamers. Thus, the affinity of the aptamers to the column was per se smaller than those of the library. This unexpected behaviour is reasonable 
since the sequences from the library did not form a complex three-dimensional structure, while the DON-aptamers were selected on forming such a structure. Hence, they had a low $\Delta \mathrm{G}$ value and were relative self-stable, which led to a lower affinity to the column. However, eluting with DON, differences between the aptamers and the library could be observed. The negative $\triangle \mathrm{RED}$ value of the library, meaning that there was less DNA DON-dependently eluted than in the controls, is probably a statistical effect and will disappear if the repetition rate would be increased. There were 14 aptamers showing a positive $\triangle \mathrm{RED}$ and thus a DON-dependent elution. The variation was in parts very large, with one aptamer, c8, showing a significantly higher DON-dependent elution than the control. However, also the others might be reacting on DON, if the repetition rate would be increased. This can be confirmed by aggregating different aptamers. The binding assay was based on the principle that small disturbances of the complementary interaction of aptamers and capture oligonucleotide will lead to a structure-switching and thus a release. These disruptions can also be caused by external factors such as slight variations in temperature, pressure or incubation time, inducing an additional variance.

The secondary structures of the selected aptamers were different, although there were similar motifs in the 5' domains found. It has been shown in the literature, that the 3' domain is often not necessary for target binding, as the selection mechanism was only focused on the 5' region [145]. However, modifications with the exclusion of this region did not show any improvements. In contrary, the performance was much worse. It indicates that this region might be involved in the tertiary structure and is essential for DON binding, as ligand recognition of aptamers is often due to the enclosure of large parts by the nucleic acid [218]. Particularly, the aptamer c8 differs from the others in terms of the secondary structure. It is furthermore relative unstable with a high $\Delta \mathrm{G}$ value. In contrast, other aptamers, e.g. c77, are highly stable with a clear defined structure. It has to be taken into account, that the structure-forming algorithm does not include the fluorophore at the 5' end, the capture DNA as well as the DON molecule the aptamer is bound to. A consideration would be, that a less stable sequence is floating between different states until the target stabilises it. Probably are the stable structures not as stable as the algorithm estimates, if the fluorophore and 


\section{DISCUSSION}

the tertiary structure would be considered.

In many of the high eluting aptamers, the common motif covers a single-base bulge. Höhn [206] supposed single-base bulges as binding caves. These small caves could probably act as binding site for DON as they offer the ideal size of about one nucleotide. This would also explain, why the binding capability was drastically decreasing as soon as the bulge was closed in the modification c $32 \mathrm{~m} 2$.

\subsection{Conclusions}

In this work it is shown that the metalloporphyrin-based Enose can be used to qualitatively detect and correctly classify dry, whole, Fusarium-infected wheat grain. Even low-contaminated grain were accurately detected, allowing them to be excluded from the food or feed chain. The Enose meets most analytical requirements needed. It is a mobile, inexpensive and relatively fast electronic device, capable of differentiating hazardous grain from innocuous grain. The fact that all classification rates were higher than $83 \%$, most of them being much higher, clearly indicated that chemometrical fingerprints allow the detection of fungal infestation as well as the discrimination between specific Fusaria species. As meanwhile specific biomarkers for Fusarium infestation are published, metalloporphyrins can be screened on these particular volatiles and assembled in an electronic nose for future improvement. However, an Enose approach for the detection of mycotoxins would always be semi-quantitative and is based on the correlation of fungal biomass and toxin content, as most of the mycotoxins are not volatile and thus could not be sniffed directly. Hence, aptamers binding DON were generated. These aptamers could be used for direct estimation of DON. Furthermore, the underlying selection mechanism has the advantage to enhance the reaction of a potential biosensor by using the capture DNA as an actor for either fluorescence modification or mass-change enhancement. DON aptamers as recognition element on biosensors have several advantages. As they provide different binding characteristics, the sensor adapts automatically to drift and to different working ranges. They are reverse denaturable, which leads to an ease of regeneration. Finally, the production is cheap and fast, once the sequences are known. In practical terms, signals, either from Fusarium or DON must be recog- 
nised in the presence of a background of interfering molecular species. This would commonly be achieved by increasing the number of sensors. Moreover, a single sensor might be not very sensitive, but the combination of sensors with different affinities and redundancy in a sensory network would dramatically enhance the robustness of such a system, since it imitates the mechanisms of natural olfaction. 


\section{Chapter 5}

\section{Summary}

F. graminearum and F. culmorum are known mycotoxin producers. They are the most pathogenic and most frequently occurring Fusaria species in cereals. Particularly, the metabolite deoxynivalenol has hazardous effects. A potential pass-through to the feed or food chain is most likely and thus, represents a serious health risk to humans. Since the NIRS technique for quality-estimation of grain has been accepted in the grain-processing industry, important quality-defining ingredients (e.g. water or protein content) are measured at the time of delivery by the farmers to add additional charges or separate the grain to different stockpiles. Particularly, the toxin content is, if at all, at that point only roughly estimated by visual inspection due to a deficiency of proper detection methods.

A widely spread method to detect fungal spoilage on food is the use of smell, which is consequently described as musty or mouldy. The use of an electronic nose for an objective analysis on the existence of fungal contamination is obvious. In this thesis, the fungal volatiles released by Fusarium graminearum, Fusarium culmorum, Fusarium cerealis and Fusarium redolens were analysed. Besides, the discriminating properties for these potentially risky Fusarium species should be investigated by the use of a metalloporphyrin-coated electronic nose at the postharvest level. Therefore, whole wheat kernels were artificially inoculated with four different Fusarium species and three incubation times. The kernels were dried to a physiologic post-harvest moisture level. The odour analysis revealed a complex composition of different compounds, among which many known fungal volatiles such as 1-octen-3-ol or 3-methyl-butanol. The electronic nose was able to 
differentiate between the different species with an accuracy of $94 \%$ and between the infection levels with $91 \%$. Furthermore, it could distinguish infested grain from non-infested ones with a correct classification rate of $83 \%$.

Motivated by these findings, a method for direct detection of deoxynivalenol should be developed. Therefore, a novel DNA-ligand system was used to select aptamers binding DON. The applied systematic evolution of ligands by exponential enrichment (SELEX) was based on the formation of aptamer beacons upon DON binding. A single-stranded, random DNA library comprising $6.6 \times 10^{14}$ different molecules was reversibly bound to an affinity column. Sequences which underwent a conformational change upon DON addition were collected and amplified. After 12 rounds of selection, the enriched pool was analysed on the included sequences. Out of 76 sequences, 39 were chosen for a detailed binding analysis. Among these, 14 reacted to the application of DON, with one (c8) eluting a significantly higher amount of DNA than the control. 


\section{Bibliography}

[1] D. W. Parry, P. Jenkinson, and L. McLeod. "Fusarium ear blight (scab) in small grain cereals; a review”. In: Plant Pathology 44.2 (1995), pp. 207-238.

[2] S. Yazar and G. Omurtag. "Fumonisins, trichothecenes and zearalenone in cereals". In: International Journal of Molecular Sciences 9.11 (2008), pp. 2062-2090.

[3] P. Kirk, P. Cannon, D. Minter, and J. Stalpers. Ainsworth \&3 bisby's dictionary of the fungi. 10th ed. Wallingford, UK: CAB INTERNATIONAL, 2008.

[4] H. F. Link. "Observationes in ordines plantarum naturales." In: Magazin für die neuesten Endeckungen in der gesammten Naturkunde. Vol. 3. Berlin: Realschulbuchhandlung, 1809, pp. 3-42.

[5] H. W. Wollenweber and O. A. Reinking. Die Fusarien ihre Beschreibung, Schadwirkung und Bekämpfung. Berlin: Paul Parey, 1935.

[6] W. Gerlach and H. Nirenberg. The genus Fusarium-a pictorial atlas. Mitteilungen aus der Biologischen Bundesanstalt für Land- und Forstwirtschaft Berlin-Dahlem 209. Berlin: Paul Parey, 1982.

[7] A. Bottalico and G. Perrone. "Toxigenic Fusarium species and mycotoxins associated with head blight in small-grain cereals in europe". In: European Journal of Plant Pathology 108.7 (2002), pp. 611-624.

[8] A. W. Schaafsma, L. Tamburic-Ilinic, J. D. Miller, and D. C. Hooker. "Agronomic considerations for reducing deoxynivalenol in wheat grain". In: Canadian Journal of Plant Pathology 23.3 (2001), pp. 279-285.

[9] Y. Wang. "Epidemiology and management of wheat scab in china". In: Fusarium Head Scab: Global Status and Future Prospects. Ed. by H. Dubin, L. Gilchrist, J. Reeves, and A. McNab. Mexico: CIMMYT, 1997, pp. 97-105.

[10] W. E. Nganje, D. A. Bangsund, F. L. Leistritz, W. W. Wilson, and N. M. Tiapo. "Regional economic impacts of Fusarium head blight in wheat and barley". In: Review of Agricultural Economics 26.3 (2004), pp. 332-347. 


\section{BIBLIOGRAPHY}

[11] G. P. Munkvold. "Epidemiology of Fusarium diseases and their mycotoxins in maize ears". In: Epidemiology of Mycotoxin Producing Fungi. Ed. by X. Xu, J. A. Bailey, and B. M. Cooke. Springer Netherlands, 2003, pp. 705-713.

[12] B. Koehler. Corn ear rots in Illinois. Vol. 639. Bulletin Series Report. Illinois: University of Illinois, 1959.

[13] J. Chelkowski. "Distribution of Fusarium species and their mycotoxins in cereal grains". In: Mycotoxins in agriculture and food safety. Ed. by K. Sinha and D. Bhatnagar. Vol. 15. 5. New York: Marcel Dekker Inc., 1998, pp. 45-66.

[14] J. P. Rheeder, W. F. Marasas, and H. F. Vismer. "Production of fumonisin analogs by Fusarium species". In: Applied and Environmental Microbiology 68.5 (2002), pp. 21012105 .

[15] S. Bäse, F. Gläser, C. Kramer, S. Lindner, A. M. Linsenmeier, K.-S. Masters, A. C. Meister, B. M. Ruff, and S. Zhong. The chemistry of mycotoxins. Ed. by A. Kinghorn, H. Falk, and J. Kobayashi. Progress in the Chemistry of Organic Natural Products 97. Wien: Springer, 2013.

[16] W. F. Marasas, T. Kellerman, W. C. Gelderblom, J. Coetzer, P. Thiel, and J. van der Lugt. "Leukoencephalomalacia in a horse induced by fumonisin B1 isolated from Fusarium moniliforme". In: The Onderstepoort Journal of Veterinary Research 55.4 (1988), pp. 197-203.

[17] P. F. Ross, L. G. Rice, R. D. Plattner, G. D. Osweiler, T. M. Wilson, D. L. Owens, H. A. Nelson, and J. L. Richard. "Concentrations of fumonisin B1 in feeds associated with animal health problems". In: Mycopathologia 114.3 (1991), pp. 129-135.

[18] W. M. Haschek, G. Motelin, D. K. Ness, K. S. Harlin, W. F. Hall, R. F. Vesonder, R. E. Peterson, and V. R. Beasley. "Characterization of fumonisin toxicity in orally and intravenously dosed swine". In: Mycopathologia 117.1-2 (1992), pp. 83-96.

[19] K. A. Voss, R. T. Riley, C. W. Bacon, W. J. Chamberlain, and W. P. Norred. "Subchronic toxic effects of Fusarium moniliforme and fumonisin B1 in rats and mice". In: Natural Toxins 4.1 (1996), pp. 16-23.

[20] K. A. Voss, R. T. Riley, W. P. Norred, C. W. Bacon, F. I. Meredith, P. C. Howard, R. D. Plattner, T. F. Collins, D. K. Hansen, and J. K. Porter. "An overview of rodent toxicities: liver and kidney effects of fumonisins and Fusarium moniliforme". In: Environmental Health Perspectives 109.Suppl 2 (2001), pp. 259-266.

[21] W. F. Marasas, K. Jaskiewicz, F. S. Venter, and D. J. Van Schalkwyk. "Fusarium moniliforme contamination of maize in oesophageal cancer areas in Transkei". In: South African Medical Journal 74.3 (1988), pp. 110-114.

[22] C. S. Yang. "Research on esophageal cancer in China: a review". In: Cancer Research 40.8 Pt 1 (1980), pp. 2633-2644. 


\section{BIBLIOGRAPHY}

[23] W. C. Gelderblom, N. P. Kriek, W. F. Marasas, and P. G. Thiel. "Toxicity and carcinogenicity of the Fusarium moniliforme metabolite, fumonisin B1, in rats". In: Carcinogenesis 12.7 (1991), pp. 1247-1251.

[24] IARC. Some naturally occurring substances: food items and constituents, heterocyclic aromatic amines and mycotoxins. IARC Monographs on the Evaluation of Carcinogenic Risks to Humans Vol. 56. World Health Organization, 1993.

[25] M. Stob, R. S. Baldwin, J. Tuite, F. N. Andrews, and K. G. Gillette. "Isolation of an anabolic, uterotrophic compound from corn infected with Gibberella zeae". In: Nature 196.4861 (1962), pp. 1318-1318.

[26] W. H. Urry, H. L. Wehrmeister, E. B. Hodge, and P. H. Hidy. "The structure of zearalenone". In: Tetrahedron Letters 7.27 (1966), pp. 3109-3114.

[27] A. Zinedine, J. M. Soriano, J. C. Moltó, and J. Mañes. "Review on the toxicity, occurrence, metabolism, detoxification, regulations and intake of zearalenone: an oestrogenic mycotoxin". In: Food and Chemical Toxicology 45.1 (2007), pp. 1-18.

[28] Y. Ueno. "The toxicology of mycotoxins". In: CRC Critical Reviews in Toxicology 14.2 (1985), pp. 99-132.

[29] J. Grove. "The trichothecenes and their biosynthesis". In: Progress in the Chemistry of Organic Natural Products. Ed. by W. Herz, H. Falk, and G. W. Kirby. Vol. 88. Wien: Springer, 2007, pp. 63-130.

[30] J. J. Pestka. "Deoxynivalenol: toxicity, mechanisms and animal health risks". In: Animal Feed Science and Technology 137.3 (2007), pp. 283-298.

[31] S. P. McCormick, A. M. Stanley, N. A. Stover, and N. J. Alexander. "Trichothecenes: from simple to complex mycotoxins". In: Toxins 3.7 (2011), pp. 802-814.

[32] G. Freeman and R. Morrison. "Trichothecin: an antifungal metabolic product of Trichothecium roseum link". In: Nature 162.4105 (1948), p. 30.

[33] R. Schoental, A. Z. Joffe, and B. Yagen. "Cardiovascular lesions and various tumors found in rats given T-2 toxin, a trichothecene metabolite of Fusarium". In: Cancer Research 39.6 Part 1 (1979), pp. 2179-2189.

[34] B. B. Jarvis, G. P. Stahly, G. Pavanasasivam, and E. P. Mazzola. "Antileukemic compounds derived from the chemical modification of macrocyclic trichothecenes. 1. derivatives of verrucarin A". In: Journal of Medicinal Chemistry 23.9 (1980), pp. 1054-1058.

[35] W. M. Haschek and V. R. Beasley. "Trichothecene mycotoxins". In: Handbook of Toxicology of Chemical Warfare Agents. Ed. by R. Cupta. Oxford: Elsevier, 2009, pp. 353369.

[36] J. Schnürer, J. Olsson, and T. Börjesson. "Fungal volatiles as indicators of food and feeds spoilage". In: Fungal Genetics and Biology 27.2-3 (1999), pp. 209-217. 


\section{BIBLIOGRAPHY}

[37] J. Kesselmeier and M. Staudt. "Biogenic volatile organic compounds (VOC): an overview on emission, physiology and ecology". In: Journal of Atmospheric Chemistry 33.1 (1999), pp. 23-88.

[38] A.-L. Pasanen, S. Lappalainen, and P. Pasanen. "Volatile organic metabolites associated with some toxic fungi and their mycotoxins". In: The Analyst 121.12 (1996), pp. 19491953.

[39] A. Korpi, J. Jörnberg, and A. L. Pasanen. The nordic expert group for criteria documentation of health risks from chemicals: 138. microbial volatile organic compounds (MVOCs). National Institute for Working Life, 2006.

[40] E. Kaminśki, S. Stawicki, and E. Wąsowicz. "Volatile flavor compounds produced by molds of Aspergillus, Penicillium and fungi imperfecti". In: Applied and Environmental Microbiology 27.6 (1974), pp. 1001-1004.

[41] T. Börjesson, U. Stöllman, P. Adamek, and A. Kaspersson. "Analysis of volatile compounds for detection of molds in stored cereals". In: Cereal Chemistry 66.4 (1989), pp. 300-304.

[42] J. Eifler, E. Martinelli, M. Santonico, R. Capuano, D. Schild, and C. Di Natale. "Differential detection of potentially hazardous Fusarium species in wheat grains by an electronic nose". In: PLoS ONE 6.6 (2011), e21026.

[43] E.-M. Becker. "New strategies for the detection of Fusarium infection and mycotoxin contamination of cereals and maize". PhD thesis. Göttingen: Georg-August Universität Göttingen, 2013.

[44] H. H. Jeleń, C. J. Mirocha, E. Wąsowicz, and E. Kaminśki. "Production of volatile sesquiterpenes by Fusarium sambucinum strains with different abilities to synthesize trichothecenes". In: Applied and Environmental Microbiology 61.11 (1995), pp. 38153820 .

[45] J. Perkowski, M. Buśko, J. Chmielewski, T. Góral, and B. Tyrakowska. "Content of trichodiene and analysis of fungal volatiles (electronic nose) in wheat and triticale grain naturally infected and inoculated with Fusarium culmorum". In: International Journal of Food Microbiology 126.1 (2008), pp. 127-134.

[46] H. Jeleń, D. Latus-Ziętkiewicz, E. Wąsowicz, and E. Kamiński. "Trichodiene as a volatile marker for trichothecenes biosynthesis". In: Journal of Microbiological Methods 31.1-2 (1997), pp. 45-49.

[47] J. S. Dickschat, N. L. Brock, C. A. Citron, and B. Tudzynski. "Biosynthesis of sesquiterpenes by the fungus Fusarium verticillioides". In: ChemBioChem 12.13 (2011), pp. 2088-2095. 


\section{BIBLIOGRAPHY}

[48] J. A. Koziel, J. P. Spinhirne, J. D. Lloyd, D. B. Parker, D. W. Wright, and F. W. Kuhrt. "Evaluation of sample recovery of malodorous gases from air sampling bags, SPME, and sampling canisters". In: Journal of the Air and Waste Management Association 55 (2005), pp. 1147-1157.

[49] A.-L. Sunesson, C.-A. Nilsson, and B. Andersson. "Evaluation of adsorbents for sampling and quantitative analysis of microbial volatiles using thermal desorption-gas chromatography". In: Journal of Chromatography A 699.1-2 (1995), pp. 203-214.

[50] J. Koziel, M. Jia, and J. Pawliszyn. "Air sampling with porous solid-phase microextraction fibers". In: Analytical Chemistry 72.21 (2000), pp. 5178-5186.

[51] R. Ruan, S. Ning, A. Song, A. Ning, R. Jones, and P. Chen. "Estimation of Fusarium scab in wheat using machine vision and a neural network". In: Cereal Chemistry Journal 75.4 (1998), pp. $455-459$.

[52] R. Ruan, S. Ning, L. Luo, X. Chen, P. Chen, R. Jones, W. Wilcke, and V. Morey. "Estimation of weight percentage of scabby wheat kernels using an automatic machine vision and neural network based system". In: Transactions of the American Society of Agricultural Engineers 44.4 (2001), pp. 983-988.

[53] F. E. Dowell, M. S. Ram, and L. M. Seitz. "Predicting scab, vomitoxin, and ergosterol in single wheat kernels using near-infrared spectroscopy". In: Cereal Chemistry 76.4 (1999), pp. 573-576.

[54] S. Delwiche. "Classification of scab- and other mold-damaged wheat kernels by nearinfrared reflectance spectroscopy". In: Transactions of the American Society of Agricultural Engineers 46.3 (2003), pp. 731-738.

[55] Z. Gan, R. R. Marquardt, D. Abramson, and R. M. Clear. "The characterization of chicken antibodies raised against Fusarium spp. by enzyme-linked immunosorbent assay and immunoblotting". In: International Journal of Food Microbiology 38.2-3 (1997), pp. 191-200.

[56] S. Li, R. Marquardt, and D. Abramson. "Immunochemical detection of molds: a review". In: Journal of Food Protection 63.2 (2000), pp. 281-291.

[57] M. S. Iyer and M. A. Cousin. "Immunological detection of Fusarium species in cornmeal". In: Journal of Food Protection 66.3 (2003), pp. 451-456.

[58] J. N. Banks, R. H. Rizvi, I. Barker, J. A. Turner, S. Rahman, and B. J. Northway. "Specific monoclonal antibodies to Fusarium species and Microdochium nivale". In: Food and Agricultural Immunology 8.4 (1996), pp. 249-268.

[59] C. Brandfass. "Establishment and application of real-time PCR-based methods to study the epidemiology of Fusarium head blight". PhD thesis. Göttingen: Georg-August Universität Göttingen, 2006. 


\section{BIBLIOGRAPHY}

[60] P. Nicholson, E. Chandler, R. C. Draeger, N. E. Gosman, D. R. Simpson, M. Thomsett, and A. H. Wilson. "Molecular tools to study epidemiology and toxicology of Fusarium head blight of cereals". In: Epidemiology of Mycotoxin Producing Fungi. Ed. by X. Xu, J. A. Bailey, and B. M. Cooke. Springer Netherlands, 2003, pp. 691-703.

[61] B. Vogelstein and K. W. Kinzler. "Digital PCR". In: Proceedings of the National Academy of Sciences of the United States of America 96.16 (1999), pp. 9236-9241.

[62] D. Morisset, D. Štebih, M. Milavec, K. Gruden, and J. Žel. "Quantitative analysis of food and feed samples with droplet digital PCR". In: PLoS ONE 8.5 (2013), e62583.

[63] FAO/WHO. Evaluation of certain mycotoxins in food. Fifty-sixth report of the joint FAO/WHO expert committee on food additives. Vol. 906. World Health Organization technical report series, 2002.

[64] N. Morooka, N. Uratsuji, T. Yoshizawa, and H. Yamamoto. "Studies on the toxic substances in barley infected with Fusarium spp." In: Food Hygiene and Safety Science 13.5 (1972), pp. 368-375.

[65] T. Yoshizawa and N. Morooka. "Deoxynivalenol and its monoacetate: New mycotoxins from Fusarium roseum and moldy barley". In: Agricultural and Biological Chemistry 37.12 (1973), pp. 2933-2934.

[66] R. F. Vesonder, A. Ciegler, and A. H. Jensen. "Isolation of the emetic principle from Fusarium-infected corn”. In: Applied Microbiology 26.6 (1973), pp. 1008-1010.

[67] SCF. Opinion on fuarium toxins. Part 1: deoxynivalenol. Scientific Committee on Food, 1999.

[68] T. Yoshizawa, H. Takeda, and T. Ohi. "Structure of a novel metabolite from deoxynivalenol, a trichothecene mycotoxin, in animals". In: Agricultural and Biological Chemistry 47.9 (1983), pp. 2133-2135.

[69] B. Kollarczik, M. Gareis, and M. Hanelt. "In vitro transformation of the Fusarium mycotoxins deoxynivalenol and zearalenone by the normal gut microflora of pigs". In: Natural Toxins 2.3 (1994), pp. 105-110.

[70] C. Keese, U. Meyer, H. Valenta, M. Schollenberger, A. Starke, I.-A. Weber, J. Rehage, G. Breves, and S. Dänicke. "No carry over of unmetabolised deoxynivalenol in milk of dairy cows fed high concentrate proportions". In: Molecular Nutrition \& Food Research 52.12 (2008), pp. 1514-1529.

[71] P. C. Turner, R. P. Hopton, Y. Lecluse, K. L. M. White, J. Fisher, and P. Lebailly. "Determinants of urinary deoxynivalenol and de-epoxy deoxynivalenol in male farmers from Normandy, France". In: Journal of Agricultural and Food Chemistry 58.8 (2010), pp. 5206-5212. 
[72] R. J. Cole and R. H. Cox. Handbook of toxic fungal metabolites. New York: Academic Press, 1981.

[73] J. H. Forsell, R. Jensen, J. H. Tai, M. Witt, W. S. Lin, and J. J. Pestka. "Comparison of acute toxicities of deoxynivalenol (vomitoxin) and 15-acetyldeoxynivalenol in the B6C3F1 mouse". In: Food and Chemical Toxicology 25.2 (1987), pp. 155-162.

[74] G. A. Bennet and O. A. Shotwell. "Criteria for determining purity of Fusarium mycotoxins." In: Journal - Association of Official Analytical Chemists 73.2 (1990), pp. 270275 .

[75] G. Eriksen and J. Alexander, eds. Fusarium toxins in cereals - a risk assessment. Copenhagen: Nordic Council of Ministers, 1998.

[76] A. F. Rizzo, F. Atroshi, T. Hirvi, and H. Saloniemi. "The hemolytic activity of deoxynivalenol and T-2 toxin". In: Natural Toxins 1.2 (1992), pp. 106-110.

[77] J. J. Pestka. "Deoxynivalenol: mechanisms of action, human exposure, and toxicological relevance". In: Archives of Toxicology 84.9 (2010), pp. 663-679.

[78] M. S. Bonnet, J. Roux, L. Mounien, M. Dallaporta, and J.-D. Troadec. "Advances in deoxynivalenol toxicity mechanisms: the brain as a target". In: Toxins 4.11 (2012), pp. $1120-1138$.

[79] R. C. Schothorst and H. P. van Egmond. "Report from SCOOP task 3.2.10 "collection of occurrence data of Fusarium toxins in food and assessment of dietary intake by the population of EU member states": subtask: trichothecenes". In: Toxicology Letters 153.1 (2004), pp. 133-143.

[80] M. Schollenberger, S. Suchy, H. Jara, W. Drochner, and H.-M. Müller. "A survey of Fusarium toxins in cereal-based foods marketed in an area of southwest Germany". In: Mycopathologia 147.1 (1999), pp. 49-57.

[81] C. M. Placinta, J. P. D'Mello, and A. M. Macdonald. "A review of worldwide contamination of cereal grains and animal feed with Fusarium mycotoxins". In: Animal Feed Science and Technology 78.1-2 (1999), pp. 21-37.

[82] C. Schwake-Anduschus and M. G. Lindhauer. "Gesundheitlich nicht erwünschte Stoffe". In: Besondere Ernte- und Qualitätsermittlung (BEE) 2013. Daten-Analysen. Bundesministerium für Ernährung, Landwirtschaft und Verbraucherschutz (BMVEL), 2013.

[83] M. Peraica, B. Radic, A. Lucic, and M. Pavlovic. "Toxic effects of mycotoxins in humans". In: Bulletin of the World Health Organization 77.9 (1999), pp. 754-766.

[84] M. Schollenberger, H. T. Jara, S. Suchy, W. Drochner, and H.-M. Müller. "Fusarium toxins in wheat flour collected in an area in southwest Germany". In: International Journal of Food Microbiology 72.1-2 (2002), pp. 85-89. 


\section{BIBLIOGRAPHY}

[85] L. González-Osnaya, C. Cortés, J. M. Soriano, J. C. Moltó, and J. Mañes. "Occurrence of deoxynivalenol and T-2 toxin in bread and pasta commercialised in Spain". In: Food Chemistry 124.1 (2011), pp. 156-161.

[86] I. Bockhorn, A. Bockhorn, and S. Pohler. "Deoxynivalenol (DON) in raw and cooked pasta". In: Mycotoxin Research 17.1 (2001), pp. 67-70.

[87] A. Kuzdraliński, E. Solarska, and M. Muszyńska. "Deoxynivalenol and zearalenone occurence in beers analysed by an enzyme-linked immunosorbent assay method". In: Food Control 29.1 (2013), pp. 22-24.

[88] M. C. Cerveró, M. A. Castillo, R. Montes, and E. Hernández. "Determination of trichothecenes, zearalenone and zearalenols in commercially available corn-based foods in Spain". In: Revista iberoamericana de micología 24.1 (2007), p. 52.

[89] H. P. Egmond and M. A. Jonker. Worldwide regulations for mycotoxins in food and feed in 2003. Food and Agriculture Organization of the United Nations, 2004.

[90] SCF. Opinion of the scientific committee on food on Fusarium toxins. Part 6: group evaluation of T-2 toxin, HT-2 toxin, nivalenol and deoxynivalenol. Scientific Committee on Food, 2002.

[91] P. C. Turner, J. A. Rothwell, K. L. White, Y. Gong, J. E. Cade, and C. P. Wild. "Urinary deoxynivalenol is correlated with cereal intake in individuals from the United Kingdom". In: Environmental Health Perspectives (2008), pp. 21-25.

[92] K. Raupach and R. Marggraf. "Unzureichender Verbraucherschutz vor dem Mykotoxin Deoxynivalnol - Aktuelle Situation und Verbesserungsmöglichkeiten". In: 52nd Annual Conference, Stuttgart, Germany, September 26-28, 2012. German Association of Agricultural Economists (GEWISOLA). 2012.

[93] FAO/WHO. Codex committee on food additives and contaminants - discussion paper on deoxynivalenol. FAO/WHO, 2002.

[94] H. Trenholm, R. Warner, and D. Prelusky. "Assessment of extraction procedures in the analysis of naturally contaminated grain products for deoxynivalenol (vomitoxin)." In: Journal - Association of Official Analytical Chemists 68.4 (1984), pp. 645-649.

[95] L. M. Cahill, S. C. Kruger, B. T. McAlice, C. S. Ramsey, R. Prioli, and B. Kohn. "Quantification of deoxynivalenol in wheat using an immunoaffinity column and liquid chromatography". In: Journal of Chromatography A 859.1 (1999), pp. 23-28.

[96] H. Valenta, S. Dänicke, and S. Döll. "Analysis of deoxynivalenol and de-epoxydeoxynivalenol in animal tissues by liquid chromatography after clean-up with an immunoaffinity column". In: Mycotoxin Research 19.1 (2003), pp. 51-55. 


\section{BIBLIOGRAPHY}

[97] S. J. MacDonald, D. Chan, P. Brereton, A. Damant, and R. Wood. "Determination of deoxynivalenol in cereals and cereal products by immunoaffinity column cleanup with liquid chromatography: interlaboratory study". In: Journal of AOAC International 88.4 (2005), pp. 1197-1204.

[98] M. W. Trucksess, S. W. Page, G. E. Wood, and T.-H. Cho. "Determination of deoxynivalenol in white flour, whole wheat flour, and bran by solid-phase extraction/liquid chromatography: interlaboratory study." In: Journal of AOAC International 81.4 (1997), pp. $880-886$.

[99] M. Klötzel, U. Lauber, and H.-U. Humpf. "A new solid phase extraction clean-up method for the determination of 12 type $\mathrm{A}$ and $\mathrm{B}$ trichothecenes in cereals and cerealbased food by LC-MS/MS". In: Molecular Nutrition 83 Food Research 50.3 (2006), pp. 261-269.

[100] B. R. Malone, C. W. Humphrey, T. R. Romer, and J. L. Richard. "One-step solid-phase extraction cleanup and fluorometric analysis of deoxynivalenol in grains." In: Journal of AOAC International 81.2 (1997), pp. 448-452.

[101] E. Razzazi-Fazeli, J. Böhm, and W. Luf. "Determination of nivalenol and deoxynivalenol in wheat using liquid chromatography/mass spectrometry with negative ion atmospheric pressure chemical ionisation". In: Journal of Chromatography A 854.1-2 (1999), pp. 45-55.

[102] R. D. Plattner. "HPLC/MS analysis of Fusarium mycotoxins, fumonisins and deoxynivalenol". In: Natural Toxins 7.6 (1999), pp. 365-370.

[103] T. Tanaka, A. Yoneda, S. Inoue, Y. Sugiura, and Y. Ueno. "Simultaneous determination of trichothecene mycotoxins and zearalenone in cereals by gas chromatography-mass spectrometry". In: Journal of Chromatography A 882.1-2 (2000), pp. 23-28.

[104] J. Olsson, T. Börjesson, T. Lundstedt, and J. Schnürer. "Detection and quantification of ochratoxin A and deoxynivalenol in barley grains by GC-MS and electronic nose". In: International Journal of Food Microbiology 72.3 (2002), pp. 203-214.

[105] A. W. Schaafsma, R. W. Nicol, M. E. Savard, R. C. Sinha, L. M. Reid, and G. Rottinghaus. "Analysis of Fusarium toxins in maize and wheat using thin layer chromatography". In: Mycopathologia 142 (1998), pp. 107-113.

[106] M. M. Abouzied, J. I. Azcona, W. E. Braselton, and J. J. Pestka. "Immunochemical assessment of mycotoxins in 1989 grain foods: evidence for deoxynivalenol (vomitoxin) contamination." In: Applied and Environmental Microbiology 57.3 (1991), pp. 672-677.

[107] R. Krska, S. Baumgartner, and R. Josephs. "The state-of-the-art in the analysis of type-A and -B trichothecene mycotoxins in cereals". In: Fresenius' Journal of Analytical Chemistry 371.3 (2001), pp. 285-299. 


\section{BIBLIOGRAPHY}

[108] R. Schuhmacher, R. Krska, J. Weingaertner, and M. Grasserbauer. "Interlaboratory comparison study for the determination of the Fusarium mycotoxins deoxynivalenol in wheat and zearalenone in maize using different methods". In: Fresenius' Journal of Analytical Chemistry 359.6 (1997), pp. 510-515.

[109] J. Hartman. "A possible objective method for the rapid estimation of flavors in vegetables". In: Proceedings of the American Society for Horticultural Science. Vol. 64. American Society for Horticultural Science. 1954, pp. 335-342.

[110] W. F. Wilkens and J. D. Hartman. "An electronic analog for the olfactory processes". In: Journal of Food Science 29.3 (1964), pp. 372-378.

[111] K. Persaud and G. Dodd. "Analysis of discrimination mechanisms in the mammalian olfactory system using a model nose". In: Nature 299.5881 (1982), pp. 352-355.

[112] J. W. Gardner and P. N. Bartlett. "A brief history of electronic noses". In: Sensors and Actuators B: Chemical 18.1-3 (1994), pp. 210-211.

[113] T. V. Getchell. "Functional properties of vertebrate olfactory receptor neurons". In: Physiological Reviews 66.3 (1986), pp. 772-818.

[114] A. Hulanicki, S. Glab, and F. Ingman. "Chemical sensors: definitions and classification". In: Pure and Applied Chemistry 63.9 (1991).

[115] P. Gründler. Chemische Sensoren - eine Einführung für Naturwissenschaftler und Ingenieure. Berlin: Springer, 2004.

[116] J. Gardner and P. Bartlett. Electronic noses: principles and applications. Vol. 67. Oxford University Press, 1999.

[117] Figaro Gas Sensors TGS. Figaro technical reference. Figaro Engineering Inc. Osaka, 2004 .

[118] G. Sauerbrey. "Verwendung von Schwingquarzen zur Wägung dünner Schichten und zur Mikrowägung”. In: Zeitschrift für Physik A Hadrons and Nuclei 155.2 (1959), pp. 206222 .

[119] C. Di Natale, R. Paolesse, and A. D'Amico. "Metalloporphyrins based artificial olfactory receptors". In: Sensors and Actuators B: Chemical 121.1 (2007), pp. 238-246.

[120] Z. Deng and D. C. Stone. "Characterization of polymer films of pyrrole derivatives for chemical sensing by cyclic voltammetry, x-ray photoelectron spectroscopy and vapour sorption studies". In: Analyst 122.10 (1997), pp. 1129-1138.

[121] K. Haupt, K. Noworyta, and W. Kutner. "Imprinted polymer-based enantioselective acoustic sensor using a quartz crystal microbalance". In: Analytical Communications 36.11-12 (1999), pp. 391-393.

[122] J.-E. Haugen and K. Kvaal. "Electronic nose and artificial neural network". In: Meat Science 49, Supplement 1 (1998), pp. 273-286. 


\section{BIBLIOGRAPHY}

[123] J. Eifler, D. Schild, W. Lücke, and D. von Hörsten. "Development of an electronic nose for Fusarium detection in wheat." In: VDI-Berichte 2060 (2009), pp. 509-514.

[124] S. M. Scott, D. James, and Z. Ali. "Data analysis for electronic nose systems". In: Microchimica Acta 156.3-4 (2006), pp. 183-207.

[125] C. Di Natale, A. Macagnano, E. Martinelli, R. Paolesse, E. Proietti, and A. D'Amico. "The evaluation of quality of post-harvest oranges and apples by means of an electronic nose". In: Sensors and Actuators B: Chemical 78.13 (2001), pp. 26-31.

[126] M. Santonico, A. Bellincontro, D. De Santis, C. Di Natale, and F. Mencarelli. "Electronic nose to study postharvest dehydration of wine grapes". In: Food Chemistry 121.3 (2010), pp. 789-796.

[127] M. Pardo, G. Niederjaufner, G. Benussi, E. Comini, G. Faglia, G. Sberveglieri, M. Holmberg, and I. Lundstrom. "Data preprocessing enhances the classification of different brands of espresso coffee with an electronic nose". In: Sensors and Actuators B: Chemical. Proceedings of the International Symposium on Electronic Noses 69.3 (2000), pp. 397-403.

[128] T. C. Pearce, J. W. Gardner, S. Friel, P. N. Bartlett, and N. Blair. "Electronic nose for monitoring the flavour of beers". In: Analyst 118.4 (1993), pp. 371-377.

[129] R. Paolesse, A. Alimelli, E. Martinelli, C. D. Natale, A. D'Amico, M. G. D'Egidio, G. Aureli, A. Ricelli, and C. Fanelli. "Detection of fungal contamination of cereal grain samples by an electronic nose". In: Sensors and Actuators B: Chemical 119.2 (2006), pp. $425-430$.

[130] M. Falasconi, E. Gobbi, M. Pardo, M. Della Torre, A. Bresciani, and G. Sberveglieri. "Detection of toxigenic strains of Fusarium verticillioides in corn by electronic olfactory system". In: Sensors and Actuators B: Chemical 108.1-2 (2005), pp. 250-257.

[131] F. Cheli, A. Campagnoli, L. Pinotti, G. Savoini, and V. Dell'Orto. "Electronic nose for determination of aflatoxins in maize". In: Biotechnology, Agronomy, Society and Environment 13.S (2009), pp. 39-43.

[132] E. Gobbi, M. Falasconi, E. Torelli, and G. Sberveglieri. "Electronic nose predicts high and low fumonisin contamination in maize cultures". In: Food Research International 44.4 (2011), pp. 992-999.

[133] V. Lippolis, M. Pascale, S. Cervellieri, A. Damascelli, and A. Visconti. "Screening of deoxynivalenol contamination in durum wheat by MOS-based electronic nose and identification of the relevant pattern of volatile compounds". In: Food Control 37 (2014), pp. 263-271.

[134] S. A. Benner, P. Burgstaller, T. R. Battersby, and S. Jurczyk. "Did the RNA world exploit an expanded genetic alphabet?" In: The RNA world. Ed. by R. Gesteland, T. Cech, and J. Atkins. New York: Cold Spring Harbor Press, 1999, pp. 163-181. 


\section{BIBLIOGRAPHY}

[135] M. J. Fedor and E. Westhof. "Ribozymes". In: Molecular Cell 10.4 (2002), pp. 703-704.

[136] D. S. Wilson and J. W. Szostak. "In vitro selection of functional nucleic acids". In: Annual Review of Biochemistry 68.1 (1999), pp. 611-647.

[137] A. D. Ellington and J. W. Szostak. "In vitro selection of RNA molecules that bind specific ligands". In: Nature 346.6287 (1990), pp. 818-822.

[138] S. M. Nimjee, C. P. Rusconi, and B. A. Sullenger. "Aptamers to proteins". In: The Aptamer Handbook. Ed. by S. Klussmann. John Wiley \& Sons, 2006, pp. 131-166.

[139] D. Drolet et al. "Pharmacokinetics and safety of an anti-vascular endothelial growth factor aptamer (NX1838) following injection into the vitreous humor of rhesus monkeys". In: Pharmaceutical Research 17 (12 2000), pp. 1503-1510.

[140] F. Opazo, M. Levy, M. Byrom, C. Schäfer, C. Geisler, T. W. Groemer, A. D. Ellington, and S. O. Rizzoli. "Aptamers as potential tools for super-resolution microscopy". In: Nature Methods 9.10 (2012), pp. 938-939.

[141] C. Tuerk and L. Gold. "Systematic evolution of ligands by exponential enrichment: RNA ligands to bacteriophage T4 DNA polymerase". In: Science 249.4968 (1990), pp. 505510 .

[142] R. Stoltenburg, C. Reinemann, and B. Strehlitz. "FluMag-SELEX as an advantageous method for DNA aptamer selection". In: Analytical and Bioanalytical Chemistry 383.1 (2005), pp. 83-91.

[143] M. Rajendran and A. D. Ellington. "In vitro selection of molecular beacons". In: Nucleic Acids Research 31.19 (2003), pp. 5700-5713.

[144] N. Green. "Thermodynamics of the binding of biotin and some analogues by avidin". In: Biochemical Journal 101 (1966), pp. 774-780.

[145] M. Rajendran and A. Ellington. "Selection of fluorescent aptamer beacons that light up in the presence of zinc". In: Analytical and Bioanalytical Chemistry 390.4 (2008), pp. $1067-1075$.

[146] M. Meli, J. Vergne, J.-L. Décout, and M.-C. Maurel. "Adenine-aptamer complexes a bipartite RNA site that binds the adenine nucleic base". In: Journal of Biological Chemistry 277.3 (2002), pp. 2104-2111.

[147] D. Kiga, Y. Futamura, K. Sakamoto, and S. Yokoyama. "An RNA aptamer to the xanthine/guanine base with a distinctive mode of purine recognition". In: Nucleic Acids Research 26.7 (1998), pp. 1755-1760.

[148] D. E. Huizenga and J. W. Szostak. "A DNA aptamer that binds adenosine and ATP". In: Biochemistry 34.2 (1995), pp. 656-665.

[149] M. Koizumi and R. R. Breaker. "Molecular recognition of cAMP by an RNA aptamer". In: Biochemistry 39.30 (2000), pp. 8983-8992. 


\section{BIBLIOGRAPHY}

[150] M. Sassanfar and J. W. Szostak. "An RNA motif that binds ATP". In: Nature 364.6437 (1993), pp. 550-553.

[151] J. H. Davis and J. W. Szostak. "Isolation of high-affinity GTP aptamers from partially structured RNA libraries". In: Proceedings of the National Academy of Sciences 99.18 (2002), pp. 11616-11621.

[152] R. Nutiu and Y. Li. "In vitro selection of structure-switching signaling aptamers". In: Angewandte Chemie 117.7 (2005), pp. 1085-1089.

[153] I. Majerfeld and M. Yarus. "Isoleucine:RNA sites with associated coding sequences." In: $R N A 4.4$ (1998), pp. 471-478.

[154] I. Majerfeld, D. Puthenvedu, and M. Yarus. "RNA affinity for molecular L-Histidine; genetic code origins". In: Journal of Molecular Evolution 61.2 (2005), pp. 226-235.

[155] A. Geiger, P. Burgstaller, H. von der Eltz, A. Roeder, and M. Famulok. "RNA aptamers that bind L-Arginine with sub-micromolar dissociation constants and high enantioselectivity". In: Nucleic Acids Research 24.6 (1996), pp. 1029-1036.

[156] M. Famulok. "Molecular recognition of amino acids by RNA-aptamers: an L-Citrulline binding RNA motif and its evolution into an L-Arginine binder". In: Journal of the American Chemical Society 116.5 (1994), pp. 1698-1706.

[157] Y. Wang, J. Killian, K. Hamasaki, and R. R. Rando. "RNA molecules that specifically and stoichiometrically bind aminoglycoside antibiotics with high affinities". In: Biochemistry 35.38 (1996), pp. 12338-12346.

[158] M. Kwon, S. Chun, S. Jeong, and J. Yu. "In vitro selection of RNA against kanamycin B". In: Molecules and Cells 11.3 (2001), pp. 303-11.

[159] S. T. Wallace and R. Schroeder. "In vitro selection and characterization of streptomycinbinding RNAs: recognition discrimination between antibiotics". In: RNA 4.1 (1998), pp. $112-123$.

[160] X. Ma, W. Wang, X. Chen, Y. Xia, S. Wu, N. Duan, and Z. Wang. "Selection, identification, and application of aflatoxin B1 aptamer". In: European Food Research and Technology (2014), pp. 1-7.

[161] J. A. Cruz-Aguado and G. Penner. "Determination of ochratoxin A with a DNA aptamer". In: Journal of Agricultural and Food Chemistry 56.22 (2008), pp. 10456-10461.

[162] M. McKeague. "Use and improvement of the systematic evolution of ligands by exponential enrichment (SELEX) process to develop aptamers for mycotoxins and other small molecules". PhD thesis. Otawa, Ontario: Carleton University, 2012.

[163] M. McKeague, C. R. Bradley, A. D. Girolamo, A. Visconti, J. D. Miller, and M. C. DeRosa. "Screening and initial binding assessment of fumonisin B1 aptamers". In: International Journal of Molecular Sciences 11.12 (2010), pp. 4864-4881. 


\section{BIBLIOGRAPHY}

[164] H. Ueyama, M. Takagi, and S. Takenaka. "A novel potassium sensing in aqueous media with a synthetic oligonucleotide derivative. Fluorescence resonance energy transfer associated with guanine quartet-potassium ion complex formation". In: Journal of the American Chemical Society 124.48 (2002), pp. 14286-14287.

[165] H. P. Hofmann, S. Limmer, V. Hornung, and M. Sprinzl. "Ni2+-binding RNA motifs with an asymmetric purine-rich internal loop and a G-A base pair." In: $R N A 3.11$ (1997), pp. 1289-1300.

[166] C. Mannironi, A. Di Nardo, P. Fruscoloni, and G. P. Tocchini-Valentini. "In vitro selection of dopamine RNA ligands". In: Biochemistry 36.32 (1997), pp. 9726-9734.

[167] C. Wilson, J. Nix, and J. Szostak. "Functional requirements for specific ligand recognition by a biotin-binding RNA pseudoknot". In: Biochemistry 37.41 (1998), pp. 1441014419.

[168] M. N. Stojanovic, P. de Prada, and D. W. Landry. "Aptamer-based folding fluorescent sensor for cocaine". In: Journal of the American Chemical Society 123.21 (2001), pp. $4928-4931$.

[169] C. T. Lauhon and J. W. Szostak. "RNA aptamers that bind flavin and nicotinamide redox cofactors". In: Journal of the American Chemical Society 117.4 (1995), pp. 12461257.

[170] A. Wochner. "Selektion von Aptameren gegen Antibiotika und deren Einsatz in empflindlichen Assayformaten". PhD thesis. Berlin: Freie Universität Berlin, 2007.

[171] A. D. Ellington and J. W. Szostak. "Selection in vitro of single-stranded DNA molecules that fold into specific ligand-binding structures". In: Nature 355.6363 (1992), pp. 850852 .

[172] D. Saran, J. Frank, and D. H. Burke. "The tyranny of adenosine recognition among RNA aptamers to coenzyme A". In: BMC Evolutionary Biology 3.1 (2003), p. 26.

[173] A. D. Keefe, S. Pai, and A. Ellington. "Aptamers as therapeutics". In: Nature Reviews Drug Discovery 9.7 (2010), pp. 537-550.

[174] E. W. M. Ng, D. T. Shima, P. Calias, E. T. Cunningham, D. R. Guyer, and A. P. Adamis. "Pegaptanib, a targeted anti-VEGF aptamer for ocular vascular disease". In: Nature Reviews Drug Discovery 5.2 (2006), pp. 123-132.

[175] Regado Biosciences. URL: http: //www.regadobio.com/ (visited on 05/04/2014).

[176] Noxxon Pharma AG. URL: http://www.noxxon.com (visited on 05/04/2014).

[177] K. P. Anderson, M. C. Fox, V. Brown-Driver, M. J. Martin, and R. F. Azad. "Inhibition of human cytomegalovirus immediate-early gene expression by an antisense oligonucleotide complementary to immediate-early RNA." In: Antimicrobial Agents and Chemotherapy 40.9 (1996), pp. 2004-2011. 
[178] E. Merki, M. J. Graham, A. E. Mullick, E. R. Miller, R. M. Crooke, R. E. Pitas, J. L. Witztum, and S. Tsimikas. "Antisense oligonucleotide directed to human apolipoprotein B-100 reduces lipoprotein(a) levels and oxidized phospholipids on human apolipoprotein B-100 particles in lipoprotein(a) transgenic mice". In: Circulation 118.7 (2008), pp. 743753.

[179] R. Paolesse, F. Mandoj, A. Marini, and C. Di Natale. "Porphyrin based chemical sensors". In: Encyclopedia of Nanoscience and Nanotechnology. Ed. by H. Nalwa. Vol. 9. Valencia CA: American Science Publishers, 2004, pp. 21-43.

[180] S. Wold, M. Sjöström, and L. Eriksson. "PLS-regression: a basic tool of chemometrics". In: Chemometrics and Intelligent Laboratory Systems 58.2 (2001), pp. 109-130.

[181] Chroma Technology Corp. URL: http://www.chroma.com (visited on 05/12/2014).

[182] Fluorophores.org. URL: http : / / www . fluorophores . tugraz . at (visited on 05/12/2014).

[183] Bio-Rad. MiniOpticon TM real-time PCR detection system. Specifications. Bio-Rad Laboratories, Inc. Hercules, CA, USA, 2009.

[184] M. Avci-Adali, A. Paul, N. Wilhelm, G. Ziemer, and H. P. Wendel. "Upgrading SELEX technology by using Lambda Exonuclease digestion for single-stranded DNA generation". In: Molecules 15.1 (2009), pp. 1-11.

[185] N. R. Markham and M. Zuker. "UNAFold: software for nucleic acid folding and hybridization". In: Bioinformatics. Ed. by J. M. Walker and J. M. Keith. Vol. 453. Totowa, NJ: Humana Press, 2008, pp. 3-31.

[186] N. Magan and P. Evans. "Volatiles as an indicator of fungal activity and differentiation between species, and the potential use of electronic nose technology for early detection of grain spoilage". In: Journal of Stored Products Research 36.4 (2000), pp. 319-340.

[187] G. Fischer, R. Schwalbe, M. Moeller, R. Ostrowski, and W. Dott. "Species-specific production of microbial volatile organic compounds (MVOC) by airborne fungi from a compost facility". In: Chemosphere 39.5 (1999), pp. 795-810.

[188] E. Kaminśki, L. M. Libbey, S. Stawicki, and E. Wąsowicz. "Identification of the predominant volatile compounds produced by Aspergillus flavus". In: Applied Microbiology 24.5 (1972), pp. 721-726.

[189] T. O. Larsen and J. C. Frisvad. "Comparison of different methods for collection of volatile chemical markers from fungi". In: Journal of Microbiological Methods 24.2 (1995), pp. 135-144.

[190] T. Nilsson, T. O. Larsen, L. Montanarella, and J. Madsen. "Application of head-space solid-phase microextraction for the analysis of volatile metabolites emitted by Penicillium species". In: Journal of Microbiological Methods 25.3 (1996), pp. 245-255. 


\section{BIBLIOGRAPHY}

[191] R. T. Marsili. "SPME-MS-MVA as an electronic nose for the study of off-flavors in milk". In: Journal of Agricultural and Food Chemistry 47.2 (1999), pp. 648-654.

[192] J.-M. Liepe. "Entwicklung und Charakterisierung von Halbleitergassensoren zur OnlineDetektion der Aromaentwicklung bei Backprozessen". PhD thesis. München: Technische Universität München, 2004.

[193] M. W. C. C. Greenshields, M. A. Mamo, N. J. Coville, A. P. Spina, D. F. Rosso, E. C. Latocheski, J. G. Destro, I. C. Pimentel, and I. A. Hümmelgen. "Electronic detection of Drechslera sp. fungi in charentais melon (Cucumis melo Naudin) using carbon-nanostructure-based sensors". In: Journal of Agricultural and Food Chemistry 60.42 (2012), pp. 10420-10425.

[194] A. Korpi, J. Järnberg, and A.-L. Pasanen. "Microbial volatile organic compounds". In: Critical Reviews in Toxicology 39.2 (2009), pp. 139-193.

[195] A.-L. Pasanen, A. Korpi, J.-P. Kasanen, and P. Pasanen. "Critical aspects on the significance of microbial volatile metabolites as indoor air pollutants". In: Environment International 24.7 (1998), pp. 703-712.

[196] H. H. Jeleń. "Use of solid phase microextraction (SPME) for profiling fungal volatile metabolites". In: Letters in Applied Microbiology 36.5 (2003), pp. 263-267.

[197] T. C. Pearce, S. S. Schiffman, H. T. Nagle, and J. W. Gardner. Handbook of machine olfaction: electronic nose technology. Weinheim: John Wiley \& Sons, 2006.

[198] S. Korsching. "Olfactory maps and odor images". In: Current Opinion in Neurobiology 12.4 (2002), pp. 387-392.

[199] C. Di Natale, D. Salimbeni, R. Paolesse, A. Macagnano, and A. D'Amico. "Porphyrinsbased opto-electronic nose for volatile compounds detection". In: Sensors and Actuators B: Chemical 65.1-3 (2000), pp. 220-226.

[200] A. D'Amico, C. Di Natale, R. Paolesse, A. Macagnano, and A. Mantini. "Metalloporphyrins as basic material for volatile sensitive sensors". In: Sensors and Actuators B: Chemical 65.1-3 (2000), pp. 209-215.

[201] C. Di Natale, J. A. J. Brunink, F. Bungaro, F. Davide, A. d'Amico, R. Paolesse, T. Boschi, M. Faccio, and G. Ferri. "Recognition of fish storage time by a metalloporphyrins-coated QMB sensor array". In: Measurement Science and Technology 7.8 (1996), p. 1103.

[202] C. Di Natale, A. Macagnano, F. Davide, A. D'Amico, R. Paolesse, T. Boschi, M. Faccio, and G. Ferri. "An electronic nose for food analysis". In: Sensors and Actuators B: Chemical 44.1-3 (1997), pp. 521-526. 
[203] P. Evans, K. C. Persaud, A. S. McNeish, R. W. Sneath, N. Hobson, and N. Magan. "Evaluation of a radial basis function neural network for the determination of wheat quality from electronic nose data". In: Sensors and Actuators B: Chemical 69.3 (2000), pp. 348-358.

[204] S. Balasubramanian, S. Panigrahi, B. Kottapalli, and C. E. Wolf-Hall. "Evaluation of an artificial olfactory system for grain quality discrimination". In: LWT - Food Science and Technology 40.10 (2007), pp. 1815-1825.

[205] L. R. Paborsky, S. N. McCurdy, L. C. Griffin, J. J. Toole, and L. L. Leung. "The singlestranded DNA aptamer-binding site of human thrombin." In: Journal of Biological Chemistry 268.28 (1993), pp. 20808-20811.

[206] B. Höhn. "Selektion und Charakterisierung von PrPSc-spezifischen Aptameren". PhD thesis. Berlin: Universität Berlin, 2006.

[207] J. Pollard, S. D. Bell, and A. D. Ellington. "Design, synthesis, and amplification of DNA pools for in vitro selection". In: Current Protocols in Nucleic Acid Chemistry. John Wiley \& Sons, Inc., 2000.

[208] R. Nutiu and Y. Li. "Aptamers with fluorescence-signaling properties". In: Methods 37.1 (2005), pp. $16-25$.

[209] P. W. Stevens, M. R. Henry, and D. M. Kelso. "DNA hybridization on microparticles: determining capture-probe density and equilibrium dissociation constants". In: Nucleic Acids Research 27.7 (1999), pp. 1719-1727.

[210] K. Tawa and W. Knoll. "Mismatching base-pair dependence of the kinetics of DNADNA hybridization studied by surface plasmon fluorescence spectroscopy". In: Nucleic Acids Research 32.8 (2004), pp. 2372-2377.

[211] A. Pyle. "Metal ions in the structure and function of RNA". In: JBIC Journal of Biological Inorganic Chemistry 7.7-8 (2002), pp. 679-690.

[212] J. M. Carothers, J. A. Goler, Y. Kapoor, L. Lara, and J. D. Keasling. "Selecting RNA aptamers for synthetic biology: investigating magnesium dependence and predicting binding affinity". In: Nucleic Acids Research 38.8 (2010), pp. 2736-2747.

[213] M. U. Musheev and S. N. Krylov. "Selection of aptamers by systematic evolution of ligands by exponential enrichment: addressing the polymerase chain reaction issue". In: Analytica Chimica Acta. Analytical Methods for Proteomics 564.1 (2006), pp. 91-96.

[214] U. B. Gyllensten and H. A. Erlich. "Generation of single-stranded DNA by the polymerase chain reaction and its application to direct sequencing of the HLA-DQA locus". In: Proceedings of the National Academy of Sciences 85.20 (1988), pp. 7652-7656. 


\section{BIBLIOGRAPHY}

[215] M. Citartan, T.-H. Tang, S.-C. Tan, and S. C. B. Gopinath. "Conditions optimized for the preparation of single-stranded DNA (ssDNA) employing lambda exonuclease digestion in generating DNA aptamer". In: World Journal of Microbiology and Biotechnology 27.5 (2011), pp. 1167-1173.

[216] J. W. Little. "An exonuclease induced by bacteriophage $\lambda$. II. Nature of the enzymatic reaction". In: Journal of Biological Chemistry 242.4 (1967), pp. 679-686.

[217] V. K. Viswanathan, K. Krcmarik, and N. P. Cianciotto. "Template secondary structure promotes polymerase jumping during PCR amplification." In: BioTechniques 27.3 (1999), pp. 508-511.

[218] J. C. Manimala. "SELEX: a tool to study the sequence specific molecular recognition of single stranded nucleic acids". PhD thesis. Austin, Texas, USA: University of Texas at Austin, 2004. 


\section{Acknowledgements}

First of all, I would like to thank my supervisors Prof. Schild and Prof. Lücke for welcoming me in their groups and for all their advice and directions they applied to me. The many discussions and the friendly encouragement were helpful throughout this thesis. It was a very joyful experience for me to be part of two completely different labs. I am very thankful for that special possibility to broaden my view on science and for the opportunity to break new ground within these research groups.

Many thanks also to Prof. Pawelzik for being a member of the examination board and evaluating this work.

I express my gratitude to the group of Prof. Di Natale from the Department of Electronic Engineering, Roma, "Tor Vergata" for the invitation to Rome and the opportunity to do the measurements with the electronic nose. Specifically, I want to thank Rosa and Eugenio for all their help and support with the analysis.

I gratefully acknowledge Christina for the many hours of lab work with the aptamers and the absorption of the setbacks.

I am very thankful to Camille and Alexander for proof-reading the manuscript and teaching me new and fancy expressions.

I would like to extend special thanks to all of my former and current colleagues. You guys made the time passing by incredibly fast by applying a friendly atmosphere during coffee sessions, movie nights, sports activities and life lessons.

Many heartfelt and sincere thanks to my parents and Christine for their support and understanding that research takes a while. 
